\title{
WestVirginiaUniversity
}

THE RESEARCH REPOSITORY @ WVU

Graduate Theses, Dissertations, and Problem Reports

2015

\section{The Role of Arginase I in Ischemic Stroke and Cardiovascular Disease}

Ashley Brooke Petrone

Follow this and additional works at: https://researchrepository.wvu.edu/etd

\section{Recommended Citation}

Petrone, Ashley Brooke, "The Role of Arginase I in Ischemic Stroke and Cardiovascular Disease" (2015). Graduate Theses, Dissertations, and Problem Reports. 6414.

https://researchrepository.wvu.edu/etd/6414

This Dissertation is protected by copyright and/or related rights. It has been brought to you by the The Research Repository @ WVU with permission from the rights-holder(s). You are free to use this Dissertation in any way that is permitted by the copyright and related rights legislation that applies to your use. For other uses you must obtain permission from the rights-holder(s) directly, unless additional rights are indicated by a Creative Commons license in the record and/ or on the work itself. This Dissertation has been accepted for inclusion in WVU Graduate Theses, Dissertations, and Problem Reports collection by an authorized administrator of The Research Repository @ WVU.

For more information, please contact researchrepository@mail.wvu.edu. 


\title{
The Role of Arginase I in Ischemic Stroke and Cardiovascular Disease
}

\author{
Ashley Brooke Petrone
}

Dissertation submitted to the West Virginia University School of Medicine in partial fulfillment of the requirements for the degree of Doctor of Philosophy in Neuroscience

\author{
Taura L. Barr, RN PhD, Chair \\ Paul D. Chantler, PhD \\ James W. Simpkins, PhD \\ Stanley M. Hileman. PhD \\ Gregory Konat. PhD
}

Department of Neuroscience

Morgantown, West Virginia

2015

Keywords: ischemic stroke, cardiovascular disease, immune system, arginase I Copyright 2015 -Ashley Petrone 


\section{ABSTRACT}

\section{The Role of Arginase I in Ischemic Stroke and Cardiovascular Disease}

\section{Ashley Brooke Petrone}

Acute ischemic stroke (AIS) is currently the fifth leading cause of death and a leading cause of long-term disability in the United States. Further, the incidence of AIS is expected to increase dramatically through year 2030. Despite the current and anticipated burden of AIS, treatment options for AIS, both acute and chronic, are extremely limited. Tissue plasminogen activator (tPA) is currently the only FDA-approved drug used to treat AIS; however, only a small fraction of AIS patients are eligible to receive tPA, and of these, only roughly 15 percent benefit from treatment with tPA. Given this, there is a crucial need to develop novel therapeutic options for AIS that may act independently or improve the efficacy of tPA. The use of human biomarker studies has led to the discovery of several biomarkers that can be used to diagnose AIS from other pathological conditions and can also be used to identify therapeutic targets for future therapeutics. Our laboratory has previously identified arginase 1 (ARG1) as the most significantly upregulated gene in the blood of AIS patients compared to control; however, the source and functional significance of this elevation is unclear. $A R G 1$ has been shown to have a broad range of functions, including mediating the immune system response and vascular function, and because changes in both immune and vascular function play a role in AIS severity and recovery, ARG1 may represent a novel prognostic marker and therapeutic target in AIS.

The purpose of this dissertation was to better characterize the role of $A R G 1$ in AIS and cardiovascular disease (CVD) as a whole. Specifically, to determine how changes in ARG1 expression may mediate immune function and vascular function, and how these alterations impact disease incidence, progression, and recovery. Herein, we have shown that increased $A R G 1$ expression is associated with changes in immune function that may contribute to post-stroke immunosuppression and poor recovery following AIS. Further, in addition to mediating the immune response to AIS, increased $A R G 1$ is also associated with increased arterial stiffness and vascular dysfunction following AIS. Lastly, we have shown that increased ARG1 expression is associated with vascular dysfunction outside of AIS, in the context of patients with CVD risk factors. Our results suggest a potential role for $A R G 1$, as both a diagnostic and prognostic marker in AIS. We also have evocative evidence to suggest that $A R G 1$ inhibition may be a potential therapeutic strategy to reduce the incidence and severity of AIS. 


\section{DEDICATION}

I would like to gladly dedicate this dissertation to both my family and friends.

To my family, thank you for being continuously supportive of me throughout my life and instilling the confidence in me to pursue my goals. I can't begin to describe what your support, both with this project and toward me in general, has helped me accomplish and make my tough days so much easier.

To my friends, thank you all for supporting me both inside and outside of the office. I hope the friendships I have formed during my time here will continue long after our time together at WVU. 


\section{ACKNOWLEDGMENTS}

I would also like to acknowledge Dr. Barr's funding sources that have made the work contained in this dissertation possible.

- Robert Wood Johnson Foundation

- National Institutes of Health COBRE 


\section{TABLE OF CONTENTS}

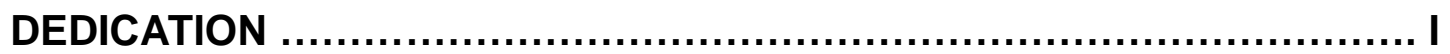

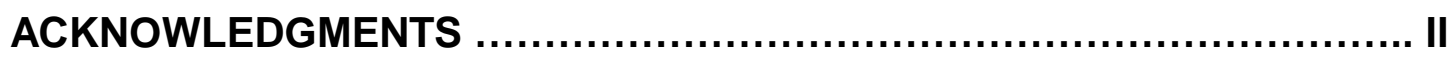

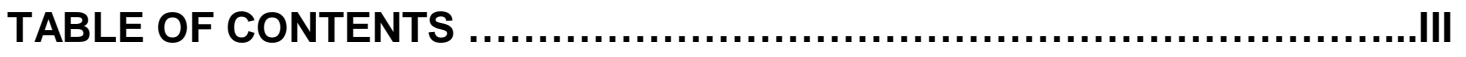

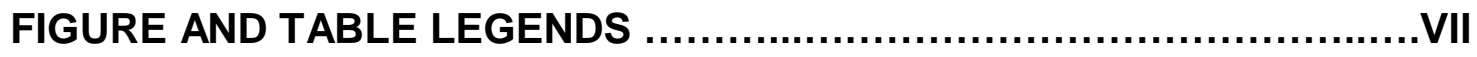

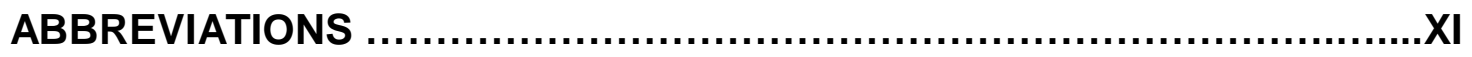

\section{CHAPTER 1. BACKGROUND AND SPECIFIC AIMS}

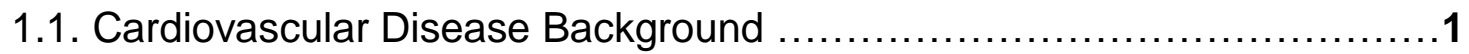

1.2. Ischemic Stroke Background ..........................................

1.3. Cardiovascular Disease and Stroke Risk Factors .........................2

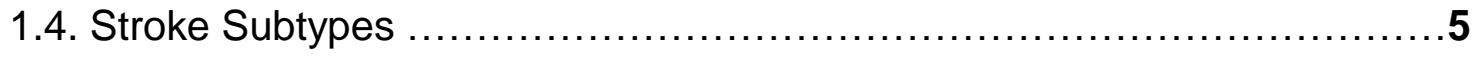

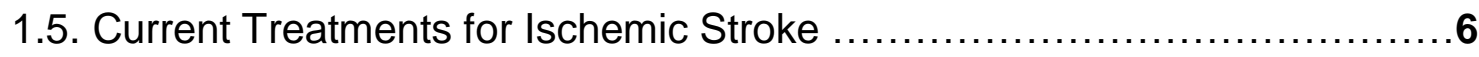

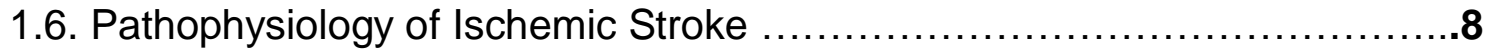

1.7. Immune Response to Ischemic Stroke ................................10

Innate Immune Response to Ischemic Stroke .........................11

Adaptive Immune Response to Ischemic Stroke .......................12

Human Biomarker Studies ..........................................15

Post-Stroke Immunosupression ......................................16

Inflammation and Risk of Stroke ....................................17

Clinical Trials Targeting the Immune System in Stroke ..................17

1.8. Neutrophil-Lymphocyte Ratio and Ischemic Stroke ...................... 18 


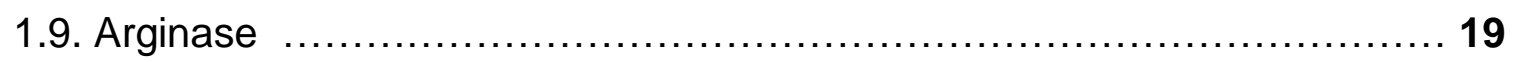

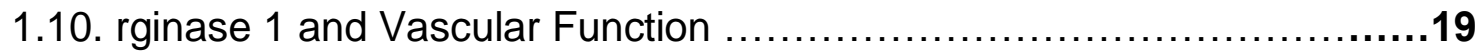

1.11. cular Function in Ischemic Stroke ....................................21

1.12. rginase 1 and Immune Function .........................................22

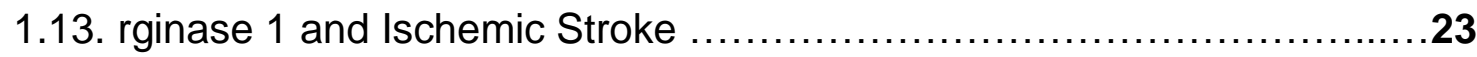

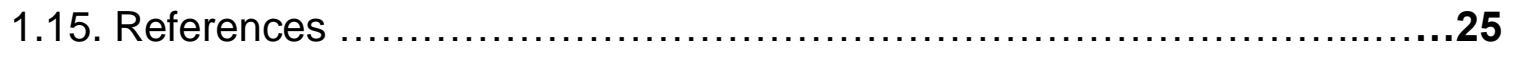

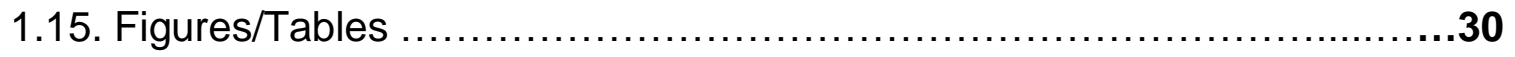

CHAPTER 2. The Role of Arginase 1 in Post-Stroke Immunosuppression and Ischemic Stroke Severity

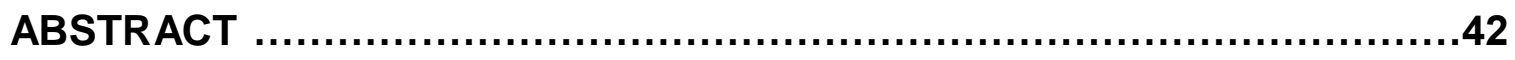

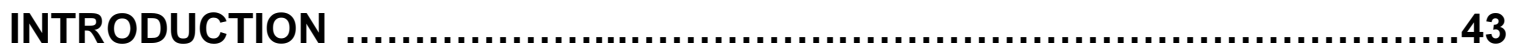

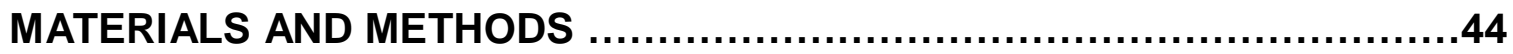

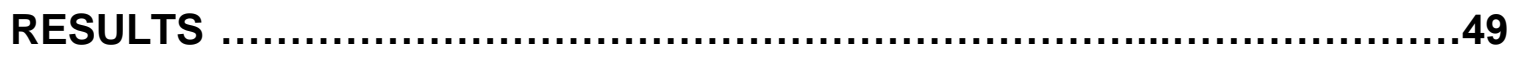

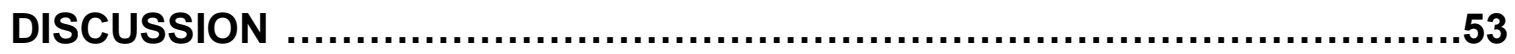

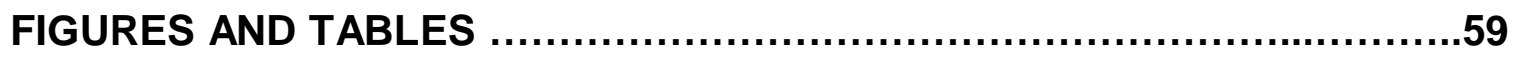

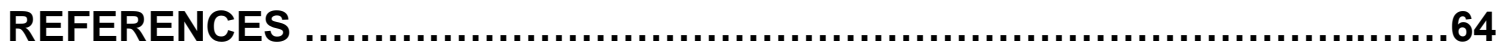


CHAPTER 3. The Relationship Between Acute Changes in Neutrophil-Lymphocyte Ratio in Ischemic Stroke Patients on Stroke Severity and Outcome

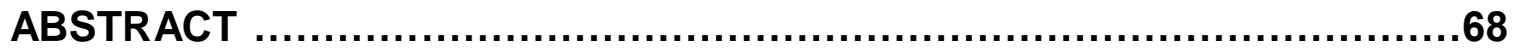

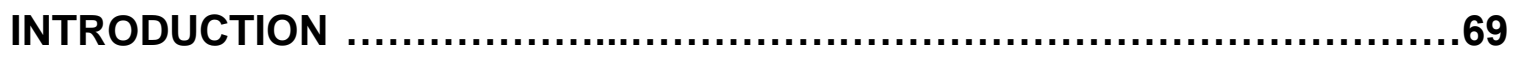

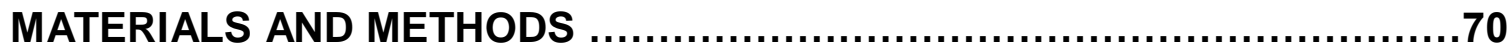

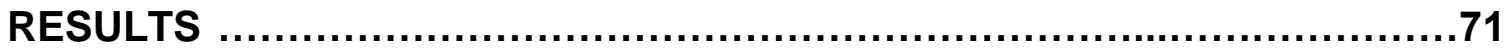

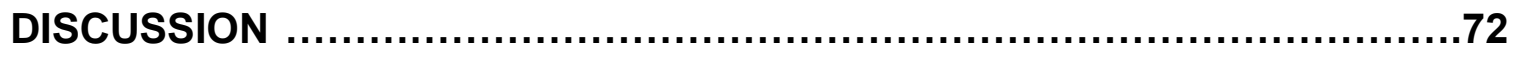

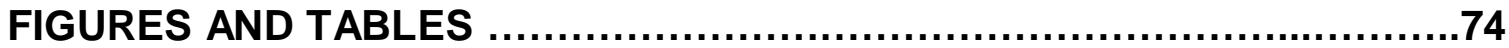

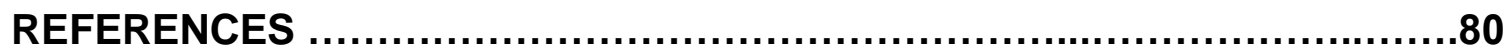

CHAPTER 4. Alterations in Vascular Function Following Ischemic Stroke in

Relation to Arginase 1 Expression

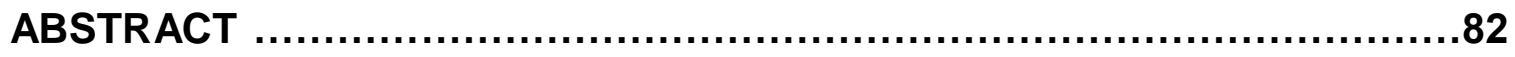

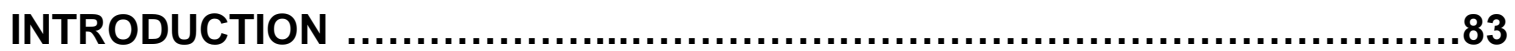

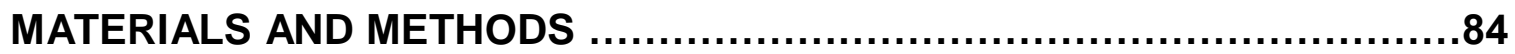

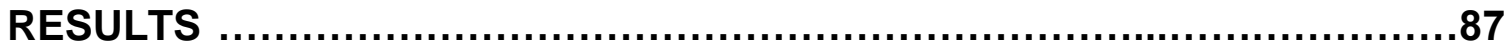

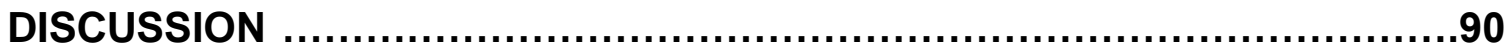

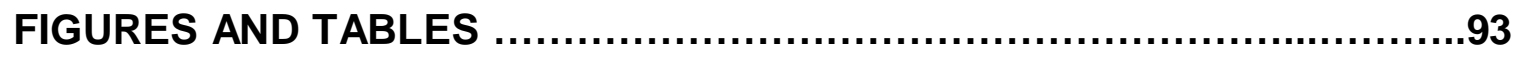

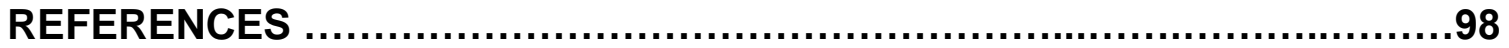


CHAPTER 5. Evaluation of the Relationship Between ARG1 Expression and Cardiovascular Function Following Lifestyle Modification

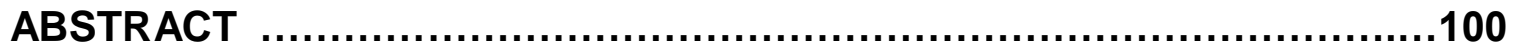

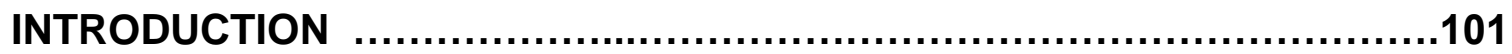

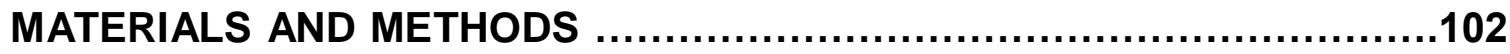

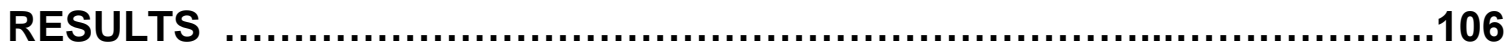

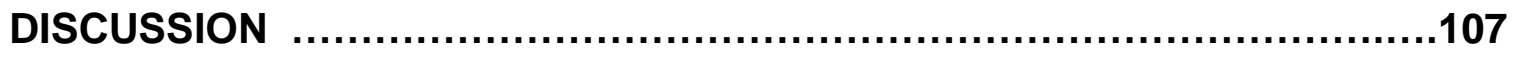

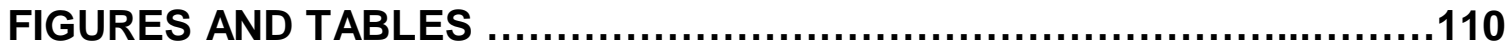

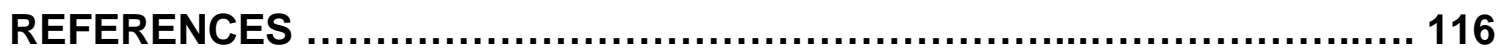

CHAPTER 6. Determination of the Cellular Origin of ARG1 Protein in Ischemic $\underline{\text { Stroke }}$

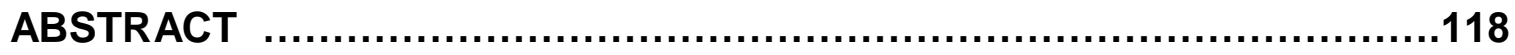

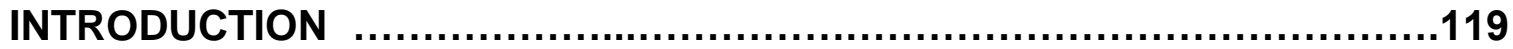

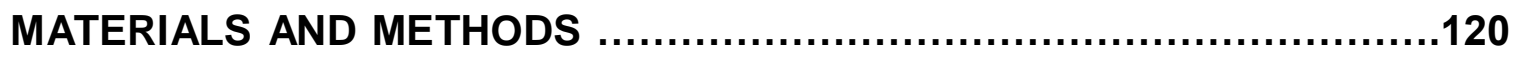

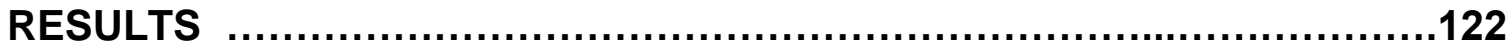

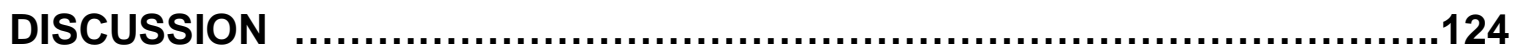

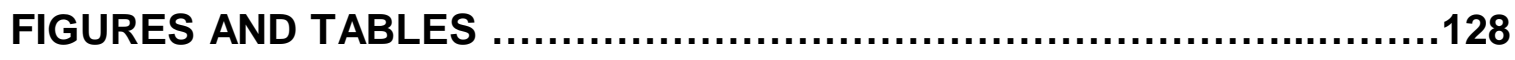

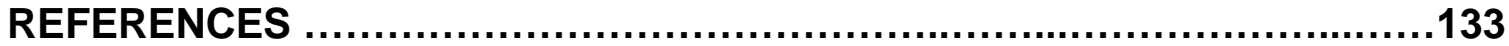




\section{CHAPTER 7. GENERAL DISCUSSION}

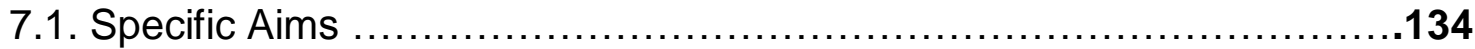

7.2. Chapter Summaries .................................................. 134

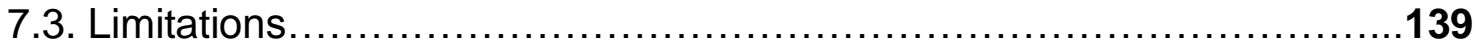

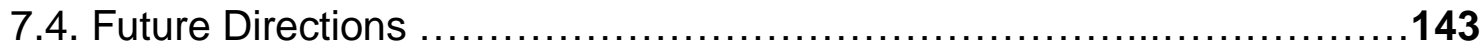

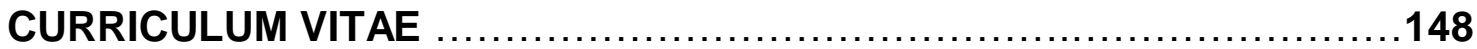




\section{FIGURE AND TABLE LEGENDS}

\section{CHAPTER 1: Introduction}

Figure 1.1: Percentage Breakdown of Deaths Attributable to Cardiovascular Disease (United States 2011).

Figure 1.2: Circle of Willis: Cerebral Vasculature Anatomy.

Figure 1.3: Cerebral Territory Supplied by the Middle Cerebral Artery.

Figure 1.4: Ischemic Cascade Following Ischemic Stroke.

Figure 1.5: Immune Response to Ischemic Stroke.

Figure 1.6: Immune-Related Genes Altered Within 24 Hours of Ischemic Stroke Compared to Control Subjects.

Figure 1.7: Leukocyte-Specific Expression of Genes Altered Within 24 Hours of Ischemic Stroke in Control Subjects.

Figure 1.8: Chronological Order of L-Arginine Metabolism by Arginase and Products.

Figure 1.9: Potential Pathways Controlling the T Cell Response to L-Arginine Metabolism.

Table 1.1: Clinical Trials Targeting the Immune Response in Ischemic Stroke.

Table 1.2: Interventional Studies with Arginase Inhibitors in Experimental and Clinical Studies.

\section{CHAPTER 2: The Role of Arginase 1 in Post-Stroke Immunosuppression and Ischemic Stroke Severity}

Figure 2.1: The Relationship between ARG1 mRNA Expression, NIHSS, and NLR.

Figure 2.2: The Relationship between Serum ARG1 Protein Activity, NIHSS, and NLR.

Figure 2.3: Relative Expression of ARG1, LY96, MMP9, s100a12, and CCR7 in AIS.

Table 2.1: Univariate Associations between Stroke and Control Subjects

Table 2.2: Loadings from Principal Component Analysis. 
CHAPTER 3: The Relationship Between Acute Changes in Neutrophil-Lymphocyte Ratio in Ischemic Stroke Patients on Stroke Severity and Outcome

Figure 3.1: The Relationship Between Baseline NIHSS and Baseline NLR.

Figure 3.2: Differences in Baseline NLR among AIS Severity Subgroups.

Figure 3.3: Differences in Baseline and 24 Hour NLR among AIS Severity Subgroups.

Figure 3.4: Change in NLR Over 24 hours $(\triangle N L R)$ in Individual Patients in AIS Severity Subgroups.

Figure 3.5: Relationship Between $\triangle \mathrm{NLR}$ and AIS Outcome.

Table 1.1: Demographic Information for Study Participants

CHAPTER 4: Alterations in Vascular Function Following Ischemic Stroke in Relation to Arginase 1 Expression

Figure 4.1: The Relationship between ARG1 Expression and AIS Severity.

Figure 4.2: Changes in ARG1 Expression in AIS Recovery.

Figure 4.3: Changes in cfPWV in AIS Recovery.

Figure 4.4: Relationship between ARG1 Expression and cfPWV in AIS Recovery.

Table 4.1: Demographic Information for Study Participants

CHAPTER 5: Evaluation of the Relationship Between ARG1 Expression and Cardiovascular Function Following Lifestyle Modification

Figure 5.1: Relationship between cIMT and cfPWV.

Figure 5.2: Relationship between cIMT and ARG1 Expression.

Figure 5.3: Relationship between cfPWV and ARG1 Expression. 
Figure 5.4: Relationship between $\triangle \mathrm{cfPWV}$ and $\triangle \mathrm{ARG1}$ Expression.

Table 5.1: Study Inclusion Criteria

Table 5.2: Demographic Information for Study Participants

\section{CHAPTER 6: Determination of the Cellular Origin of ARG1 Protein in Ischemic Stroke}

Figure 6.1: Relationship Between Whole Blood ARG1 mRNA Expression and Neutrophil ARG1 Protein in AIS and Control.

Figure 6.2: Relationship between Total Leukocyte ARG1 Protein and Whole Blood ARG1 mRNA Expression.

Figure 6.3: Intracellular ARG1 Staining in Leukocyte Subpopulations in AIS and Control Subjects.

Figure 6.4: Intracellular ARG1 Staining in Leukocyte Subpopulations in AIS Patients by Stroke Severity.

Table 6.1: Demographic Information for Stroke and Control Subjects. 


\section{LIST OF ABBREVIATIONS}

Cardiovascular Disease

CVD

Blood pressure

Acute ischemic stroke

BP

Atrial fibrillation

AIS

Tissue plasminogen activator

AF

Internal carotid artery

Anterior cerebral artery

Middle cerebral artery

TPA

Posterior cerebral artery

ICA

ACA

MCA

Cerebral blood flow

$P C A$

CBF

Blood-brain barrier

BBB

Danger-associated molecular pattern

DAMP

Matrix metalloproteinase 9

MMP9

Arginase

ARG

Middle cerebral artery occlusion

MCAO

Antigen-presenting cell

APC

Interferon-gamma

IFNy

Interleukin

IL

Helper T cell

CD4+

Cytotoxic $T$ cell

Regulatory $T$ cell

Neutrophil-Lymphocyte Ratio

CD8+

Treg

Modified Rankin Score

NLR

MRS

Nitric oxide synthase

NOS

Endothelial NOS

eNOS

Carotid-intima medial thickness

CIMT

PWV

Pulse wave velocity

Common carotid artery

CCA

Carotid-femoral PWV

cfPWV

Brachial-ankle PWV

baPWV

Lymphocyte antigen 96

LY96

S100 calcium binding protein

Chemokine CC motif receptor 7

s100a12

CCR7

NIHSS

PPIB

B2M

Beta-2 Microglobulin

iNOS 


\section{Cardiovascular Disease}

Total cardiovascular disease (CVD) is the leading cause of death, in the United States and worldwide, and roughly 1 out of 3 Americans has a degree of CVD[1]. Total CVD is defined as having at least one of the following conditions: coronary heart disease, stroke, high blood pressure, and disease of the arteries (Figure 1)[1]. While the slight majority of CVD cases occur in the population aged $>60$ years, an almost equal incidence of CVD occurs in the younger population[1]. This incidence among younger Americans contributes to the anticipated increased prevalence of CVD over the next decade. Concordant with the overall CVD prevalence and mortality rate, these increases are expected to be more dramatic in distinct populations, including rural populations and geographic areas with low socioeconomic status[1]. Aside from the high mortality rate and social burden of CVD, the financial burden associated with treating CVD is currently nearly 320 billion dollars per year and is expected to increase to 918 billion dollars annually by year 2030[1].

\section{Stroke}

Stroke represents 16 percent of total CVD cases, and approximately 6.6 million people worldwide have had a stroke, with roughly 800,000 new strokes occurring annually in the US[1]. The prevalence of stroke has been reported to range from $6-28 \%[1]$, and this wide range is likely due to both unreported cases and unclear diagnosis of stroke. Similar to CVD, the prevalence of stroke is expected to increase by 20.5 percent by year 2030[1]. When considered independent of CVD, stroke is currently the fifth leading cause of death 
in the US and second leading cause of death worldwide, and while the mortality rate of stroke has decreased over the past decade, the number of people living with stroke is expected to increase, making stroke a leading cause of long-term disability in the US[1]. Considered together, the total financial cost of treating stroke is expected to increase from the current $\$ 71.6$ billion to $\$ 184.1$ billion dollars spent annually on stroke care, and a large proportion of these funds are due to rehabilitation costs[1].

\section{Risk Factors for Cardiovascular Disease and Stroke}

Stroke is by definition a form of total CVD, thus stroke and CVD share several risk factors summarized below. Further, while each individual risk factor may contribute to AIS through different pathophysiological mechanisms, each of the following AIS risk factors has been shown to contribute to thrombus/embolus formation, vascular damage, endothelial dysfunction, atherosclerosis, and chronic inflammation. Additional mechanisms may be noted under each individual risk factor, in addition to these common

mechanisms. The role of inflammation in AIS will be discussed in more detail later in this document.

Race/Ethnicity

There are differences in both AIS prevalence and mortality rate among different ethnic groups. Specifically, AIS prevalence is higher among blacks and Hispanics compared to whites, and AIS mortality rate is highest among blacks compared to all other ethnic groups[1]. The differences between ethnic groups is likely due to an unequal distribution of CVD risk factors and socioeconomic status among the groups. 


\section{Gender}

Women have a higher overall lifetime risk of AIS than men[1]. Further, in 2014, the American Heart Association released specific guidelines for treating AIS in women that addressed several AIS risk factors that are specific to women, as well as discussed common risk factors that are more prevalent in women than men[2]. Both oral contraceptive use and preeclampsia during pregnancy have been show to increase AIS risk in women[2]. Interestingly, atrial fibrillation, an AIS risk factor common to both women and men, has been shown to confer a greater risk of AIS in women than in men[2].

\section{Hypertension}

Hypertension has been shown to be the strongest predictor of future AIS for all AIS subtypes[3]. Individuals with a BP greater than 140/90 have 2-4 times greater AIS risk than individuals with a BP $\leq 120 / 80[1]$. Further, antihypertensive medications have been shown to dramatically reduce AIS risk, and administration of antihypertensive medications following AIS reduces the risk of recurrent stroke in AIS patients[1].

\section{Diabetes Mellitus}

Individuals with increased blood glucose levels above normal are at nearly double the risk of AIS than individuals with normal glucose levels[1]. Further, the risk of AIS in individuals with uncontrolled diabetes is 3-fold higher than normal[1]. Lastly, controlling blood glucose, both acutely and after discharge following AIS, has been shown to reduce AIS severity and improve functional outcomes[1].

\section{Atrial Fibrillation}

After hypertension, atrial fibrillation (AF) is the second most common risk factor for AIS. $\mathrm{AF}$ is a condition characterized by an abnormal heart rhythm that occurs when the atria 
of the heart do not fully contract or fibrillate. This failure to contract does not allow the entire volume of blood contained in the atria to flow into the ventricles. In chronic AF, the atria fibrillate for extended periods of time causing blood to pool in the atria, which may eventually clot and be ejected into the bloodstream. Incidence of AIS in individuals with AF is nearly five times higher than normal[1].

\section{Psychosocial Risk Factors}

Recently, there has been an influx of data to suggest a relationship between psychological status and AIS risk. Specifically, generalized anxiety, depression, and other mood disorders are associated with an increased risk of AIS[1]. Further, depression is more common among AIS survivors, independent of AIS severity and disability following AIS[4].

In the Atherosclerosis Risk in Communities study (ARIC), multivariate analysis showed that age, smoking, systolic blood pressure, and diabetes mellitus were independent risk factors for all ischemic stroke subtypes[3]. Diabetes and smoking were important predictors of lacunar and non-lacunar ischemic stroke, and the risk appears to be slightly higher for lacunar compared to non-lacunar AIS[3]. The results of the ARIC study suggest that the etiologic relationship of risk factors with ischemic stroke varies by subtype; however, the molecular mechanisms giving rise to differences in AIS subtype risk are unclear.

\section{Types of Stroke}

Stroke can be broadly classified into two subtypes: acute ischemic stroke (AIS) and hemorrhagic stroke. AIS makes up approximately 87 percent of total strokes, and as 
discussed previously, the prevalence of AIS is expected to increase over the next 20 years, whereas prevalence and mortality rates associated with hemorrhagic stroke have been relatively stable in past years and no increases are expected in the near future. The focus of this dissertation is solely on AIS, and hemorrhagic stroke will not be discussed in detail; however, while the pathophysiology of AIS and hemorrhagic stroke differ, the functional deficits among stroke survivors are similar.

AIS can be further defined into more narrow subtypes based on AIS etiology, and a system of classification has been developed for the Trial of Org 10172 in Acute Stroke Treatment (TOAST)[5]. The TOAST classification identified five AIS subtypes summarized below:

\section{(1) Large-Artery Atherosclerosis (Thrombus/Embolus)}

- Defined by imaging of at least 50 percent stenosis of intra- or extra cranial vessel[5]

- Represents $\sim 27$ percent of diagnosed AIS cases[6]

(2) Cardioembolic

- Risk factors include AF or other arrhythmias, valvular disease, prosthetic valves, myocardial infarction (MI)[5]

- Represents the largest proportion of determined AIS cases - 40 percent[6]

\section{(3) Small-Vessel or Lacunar}

- Lacunar AIS is an occlusion of penetrating vessels that supply deep brain regions.

CT or MRI to confirm ischemic territory is indicative of lacunar AIS[5].

- Represents 25 percent of determined AIS cases[6] 
(4) Other Determined Cause - Probable- 4 percent of determined AIS cases[6]

(5) Unknown - Cryptogenic - 52 percent of all AIS cases[6]

The TOAST classification is the most commonly used AIS subtype classification used in the US, however, there are other subtype classification systems, such as the Causative Classification System (CCS) and the Chinese Ischemic Stroke Subclassification system (CISS), that are used to determine AIS etiology as well[7].

\section{Current Treatments for Ischemic Stroke}

For decades, tissue plasminogen activator (tPA) has been the only pharmacological thrombolytic agent available for the acute treatment of AIS and is considered the "gold standard" in AIS care[8]. Unfortunately, given intravenously, tPA achieved recanalization that leads to an improvement in AIS severity and functional outcome in only 15 percent of patients [8]. Further, due to several contraindications, only 4 percent of patients are eligible to receive intravenous tPA. Arguably, the largest limitation is the narrow time window for treatment, 4.5 hours following AIS symptom onset, that intravenous tPA has been shown to be safe and effective[8]. Recently, intra-arterial tPA has been approved for use in AIS for up to 5.5 hours after AIS symptom onset, and while intra-arterial tPA administration has been shown to be slightly more effective than intravenous tPA administration, intra-arterial tPA is also only effective in a small number of eligible patients[8]. Aside from tPA, mechanical clot retrieval has also been a therapeutic option in AIS. Recently in 2015, the results of several clinical trials evaluating the use of mechanical retrieval devices alone or in combination with intra-arterial tPA have led to a 
reevaluation of the gold standard of AIS care. In the Multicenter Randomized Clinical Trial of Endovascular Treatment for Acute Ischemic Stroke in the Netherlands (MR CLEAN), mechanical retrieval and tPA administration within 6 hours of AIS symptom onset resulted in improvements in severity and outcome that were greater than those seen with intravenous or intra-arterial tPA alone[9]. While the ability to utilize mechanical retrieval devices is limited by several factors, including clot location, eligible patients exhibit profound improvements compared to prior treatment strategies[10]. In addition to the thrombolytic approaches recently discussed, there has been a large number of drugs that have shown therapeutic efficacy in pre-clinical models of AIS. These drugs have a wide range of actions that may aid in neuroprotection, both acutely and chronically following AIS; however, most, if not all of these drugs failed in clinical trials. A summary of the most recent clinical trials in AIS and their results is summarized in Table 1.1.

\section{Ischemic Stroke Pathophysiology}

Regardless of AIS subtype, the pathophysiological mechanisms that result in brain damage are similar. Briefly, AIS is defined by a lack of blood flow to the brain, commonly due to a clot impeding blood flow in a vessel responsible for supplying blood to the brain. Reduced oxygen delivery results in brain cell death in the immediate and surrounding areas supplied by the occluded vessel. The brain is supplied by two main blood vessels: the internal carotid arteries (ICA) and the vertebral arteries. The internal carotid arteries arise as a branch of the common carotid artery in the neck and supply the anterior portion of the brain. Specifically, the ICA branches into two large branches, the anterior (ACA) and middle cerebral arteries (MCA), and these vessels supply the entire frontal cortex, as 
well as regions of the temporal and parietal cortices. The ACA and MCA further branch into smaller vessels that penetrate the cortex to supply deep brain structures, such as the basal ganglia and thalamus. The basilar artery is formed by the joining of the vertebral arteries, and the basilar artery branches once inside the skull to supply the posterior portion of the brain. Specifically, the basilar artery branches into the posterior cerebral arteries (PCA) to complete the Circle of Willis formed by the ACA, MCA, and communicating arteries (Figure 1.2). The basilar artery supplies the pons and brainstem, and the PCA supplies the posterior cerebral cortex and branches to supply the midbrain and cerebellum[11].

The specific vessel occluded in AIS will determine the distinct symptoms and degree of symptoms manifested in AIS. Approximately 70 percent of AIS are the result of occlusion of the anterior circulation (ACA and MCA), and the MCA and its branches are the most commonly occluded vessels in AIS. Further, because the MCA and branches are responsible for supplying blood to nearly 50 percent of the brain, a large region of brain is susceptible to cell death due to ischemia (Figure 1.3). Because the MCA supplies the broadest area of the cerebral cortex, AIS symptoms resulting from an occlusion of the MCA can be numerous and vary greatly.

The brain is one of the most metabolically active and demanding organs in the body, and this is reflected by the fact that while the brain is only 2 percent of total body weight, it accounts for roughly 20 percent of the body's total oxygen consumption. Further, an average weight brain requires an oxygen consumption rate of $49 \mathrm{~mL} \mathrm{O}_{2} / \mathrm{min}$ to maintain the energetic requirements required for normal brain function. This oxygen is predominantly utilized in combination with glucose to produce adenosine triphosphate 
(ATP). Both oxygen and glucose are delivered to the brain via the blood and total cerebral blood flow (CBF) must remain relatively constant at a rate of $798 \mathrm{~mL} / \mathrm{min}[12]$.

During AIS, the ischemic area has two distinct regions: the infarct core and penumbra. The infarct core is the center of the ischemic area in the immediate territory of the occluded blood vessel, and CBF in this region falls to $<10-12 \mathrm{~mL} / \mathrm{min}$. The penumbra surrounds the infarct core, and due to increased blood supply from collateral vessels, continues to receive slightly more $\mathrm{CBF}$ at a rate of $18-20 \mathrm{~mL} / \mathrm{min}$. Due to the dramatic reduction in CBF at the infarct core, neurons and glial cells in this area will begin to die within minutes of ischemic onset, and cells in the penumbra can only survive 1-2 hours if CBF is not restored[13].

If $\mathrm{CBF}$ is not rapidly restored, the ischemic cascade is activated, which is depicted in Figure 1.4. Cells in the ischemic area deplete local $\mathrm{O}_{2}$ and glucose stores and can no longer produce the amount of ATP required to function. The cells in this area then shift to anaerobic metabolism, resulting in the production of toxic bi-products, such as lactic acid, that directly damage neurons. Further, ATP depletion causes cells to fail to maintain ionic balance through channels such as the sodium/potassium pump. This induces cell death through several mechanisms, including cellular edema and excessive calcium influx, which triggers excessive neurotransmitter release or excitotoxicity, reactive oxygen species generation by mitochondria, and activation of apoptosis via mitochondrialdependent and independent mechanisms[13]. 


\section{The Immune Response and Ischemic Stroke}

Dead and dying brain cells release signals that activate both the innate and adaptive arms of the immune system following AIS, and the immune system plays a critical role in the resolution of ischemia and ischemic damage. Under normal circumstances, the brain and CNS are considered to be "immune privileged", as the CNS is kept sequestered from the immune mediators present in the periphery through maintenance of the blood-brain barrier (BBB). Brain endothelial cells, joined by tight junctions that create a barrier that is highly selective of the molecules that can enter the brain, form the BBB. Further, glial cells, specifically astrocytes, surround the endothelial cells of the BBB and provide trophic support to endothelial cells. Following stroke, both the endothelial cells and astrocytes are susceptible to death through ischemia and as these cells begin to die, the BBB becomes more permeable and the CNS becomes more exposed to the peripheral immune response. Further, reactive oxygen species generation and local immune signals in the CNS can promote BBB breakdown. The loss of BBB integrity allows for both peripheral immune cells and immune mediators, such as cytokines and chemokines, to more freely enter the brain to elicit a response. While a degree of immune cell infiltration into the brain is crucial for AIS recovery, the distribution and total amount of immune cells entering the CNS can result in both beneficial and harmful effects on AIS outcome. The innate and adaptive immune responses are summarized below, with regard to their role in AIS recovery. Figure 1.4 also summarizes ischemic cascade following AIS. 
Innate Immune Response to AIS

The innate immune response is a rapid, non-specific response that protects the body from pathogens, termed inflammation. Thus, the cells of the innate immune arm are the first to respond and/or migrate into the brain following AIS. Neutrophils are the predominant cell type of the innate arm and are also the most abundant circulating leukocyte in humans[14]. Neutrophils are responsible for phagocytosis and killing of microorganisms, and to accomplish these functions, neutrophils possess secretory granules that are released upon neutrophil activation. Dying brain cells release signals, danger-associated molecular patterns (DAMPS) that bind to receptors on neutrophils to induce their activation. Upon activation, neutrophils release their secretory granules that contain several enzymes, the most abundant of which is matrix metalloproteinase 9 (MMP9), but also contain several cytokines and chemokines including pro-inflammatory, tumor necrosis factor related apoptosis inducing ligand (TRAIL), and anti-inflammatory cytokine transforming growth factor beta (TGF $\beta$ )[14]. Neutrophil subtypes have been identified in animal studies based on their propensity to either promote or inhibit inflammation; however, no neutrophil subtypes have been identified in humans. It is important to note that arginase 1 (ARG1) mRNA expression was used as the sole marker to define proinflammatory neutrophils in animals[15].

Because of the dualistic role of neutrophils in promoting and inhibiting inflammation, the question of whether neutrophils play a beneficial or harmful role following AIS remains, and there is evidence to support both roles. Implicating neutrophils as harmful following AIS is the correlation between the time of neutrophil migration into the brain and the subsequent increase in infract size following AIS[14]. Further, in a rodent 
AIS model, administration of anti-neutrophil serum reduced infarct size following middle cerebral artery occlusion (MCAO)[16]. There is also evidence to suggest that neutrophil accumulation in the brain may directly block microvessel circulation, reducing collateral blood flow to the infarct, providing a functional mechanism of decreased infarct size as the result of reduced neutrophil infiltration into the brain [16]. To this end, several clinical trials were designed to prevent neutrophil infiltration into the brain following AIS. AIS patients in the Enlimomab Acute Stroke trial treatment group were given Enlimomab, an anti-ICAM1 antibody, which had been previously shown to decrease infarct size and reduce neutrophil infiltration into the brain in animal models of AIS. In this trial, patients treated with Enlimomab had no reductions in infarct size and patients displayed worse functional outcomes compared to the placebo group[17]. These conflicting results between animal models of AIS and human clinical trials demonstrate the complicated role of neutrophils in AIS and warrant further investigation.

\section{Adaptive Immune Response to AIS}

One of the main functions of the innate arm of the immune response is to activate the adaptive immune response. In contrast to inflammation, the adaptive immune response is directed at a specific antigen and confers a more direct resolution than innate inflammation. T and B-lymphocytes are the predominant cells of the adaptive immune response[18], and while both have been implicated in AIS, the remainder of this section will focus on the role of T lymphocytes in AIS (Figure 1.5).

Prior to initiating an immune response, $T$ cells must be activated by an antigenpresenting cell (APC). Once an APC has bound an antigen, the APC presents the antigen to the $\mathrm{T}$ cell, activates the $\mathrm{T}$ cell through costimulation via membrane bound receptors, 
and activated $\mathrm{T}$ cells can then rapidly divide and secrete cytokines to elicit an antigenspecific immune response. The two receptors/signals required for $\mathrm{T}$ cell activation are: the $\mathrm{T}$ cell receptor, CD3, located on the $\mathrm{T}$ cell membrane, and a costimulatory molecule on the surface of the APC, typically CD28[18].

Overall, lymphocytes enter the brain roughly 1-7 days following AIS[18]; however, there are several different $\mathrm{T}$ lymphocyte subsets, and each lymphocyte subset has a distinct role following AIS, which is summarized below.

\section{Helper T Lymphocytes}

Helper T lymphocytes, generally referred to as CD4+ T cells, activate other T lymphocyte subsets, specifically cytotoxic CD8+ T cells, and other immune cells[18]. CD4+ T cells can be further broken into several subtypes, including $\mathrm{TH}_{1}, \mathrm{TH}_{2}$, and $\mathrm{TH}_{17}$ helper $\mathrm{T}$ cells, based on the cytokines each subset secretes upon activation. $\mathrm{TH}_{1}$ cells are considered pro-inflammatory in nature, as interferon gamma (IFNy) is their most abundantly secreted cytokine mediator. IFNy has been shown to contribute to increased infarct volume in animal models of AIS, and increased IFNy levels are seen in human AIS patients and positively correlate with AIS severity. $\mathrm{TH}_{2}$ helper $\mathrm{T}$ cells are considered anti-inflammatory in nature, as their primary cytokine effectors are interleukin-4 (IL4) and IL-10 have been shown to have a protective effect in animal models of $\operatorname{AIS}[19,20]$. $\mathrm{TH}_{17}$ are distinct from $\mathrm{TH}_{1}$ and $\mathrm{TH}_{2}$ helper $\mathrm{T}$ cells and are named for their primary cytokine effector, IL-17. IL-17 has been shown to exacerbate damage in the late phase (3-7 days) following AIS[21]. Further, IL-17 can activate other pro-inflammatory immune cells, such as Y $\delta \mathrm{T}$ Lymphocytes that will further increase IL-17 following AIS[22]. 


\section{Yo T Lymphocytes}

Y $\delta$ T Lymphocytes are considered a hybrid between an innate and adaptive immune cell, given their ability to bind DAMPs to mediate non-specific inflammation, as well as an antigen-specific response. As mentioned previously, Yठ T Lymphocytes secrete IL-17 and like $\mathrm{TH}_{17}$ cells have been shown to exacerbate damage following AIS[22]. Further, production of IL17A has been shown to increase damage following AIS through increased neutrophil recruitment to the brain, and administration of anti-IL17A dramatically reduces infarct volume in an animal model of AIS, in part due to reduced neutrophil infiltration into the brain[22].

\section{Cytotoxic T Lymphocytes}

Cytotoxic T lymphocytes, generally referred to as CD8+ killer T cells, are the predominant effector T cell subset. Upon activation, CD8+ cells release cytotoxic enzymes to initiate apoptosis in infected or compromised cells. CD8+ cells are generally considered to be the primary $\mathrm{T}$ lymphocyte subset responsible for the detrimental effect $\mathrm{T}$ lymphocytes appear to have following AIS[23]. In support of this, mice depleted of CD8+ t cells have a dramatic reduction in infarct volume following AIS compared to wild type[23].

\section{Regulatory T Lymphocytes}

Regulatory $T$ lymphocytes ( $T_{R E G}$ ) are considered anti-inflammatory in nature due to the production of IL-10 and TGF $\beta$, and function to inhibit other immune cells, predominantly cytotoxic CD8+ T cells, to prevent autoimmune responses[24]. Because of the detrimental role of CD8+ T cells in AIS, the response of TREG cells has been implicated as a protective mechanism following AIS[24]; however, there is also conflicting evidence to suggest that 
TREG cells may have a detrimental effect following stroke by inducing cerebral microvasculature dysfunction[25].

\section{Human Biomarker Studies}

There has been an abundant amount of research focused on identifying biomarkers of AIS in human patients. Gene expression or mRNA expression in the peripheral blood of AIS patients can be used to peripherally monitor the immune response to AIS, as changes in gene expression measured in the whole blood are the result of altered gene expression in leukocytes[26]. Microarray technology allows for the analysis of total mRNA in patient whole blood and cerebrospinal fluid, providing insight into the gene expression changes related to the immune response to AIS. Not only can these changes in gene expression be used to identify leukocyte subsets that are up-regulated in blood, but also the effector genes that characterize the immune response mounted by total leukocytes. There have been several studies, by our laboratory and others, measuring gene expression in human AIS patients within 24 hours of symptom onset[27, 28]. These studies have identified immune-related genes that are significantly altered in AIS compared to control, that may represent novel therapeutic targets in AIS (Figure 1.6). Further, these gene expression changes have been examined in regards to the particular leukocyte subset responsible for the alteration seen in whole blood and have demonstrated that changes in gene expression in whole blood are mainly attributed to neutrophils and monocytes following AIS[27] (Figure 1.7). These studies provide insight into the immune response to AIS in human patients that may be translated for testing in animal models of AIS. 


\section{Post-Stroke Immunosuppression}

While AIS induces a local immune response in the brain, AIS also has a profound impact on systemic immunity. Within days of AIS, many AIS patients develop immunodepression, characterized by decreased total lymphocyte counts, splenic atrophy, and increased antiinflammatory cytokine production, collectively known as post-stroke immunosuppression[29]. While the mechanisms that induce post-stroke immunosuppression are unclear, the degree of immune suppression can greatly impact AIS recovery, as approximately 40 percent of AIS patients experience a secondary infection, commonly pneumonia or urinary tract infection, following AIS[30]. These secondary infections are associated with increased mortality and poor outcome following AIS, independent of initial AIS severity and infarct volume. The evolutionary purpose of post-stroke immunosuppression is unclear, however, it has been speculated that this response may originate in the brain to limit the autoimmune response in the brain in the delayed phase of AIS. While this may serve to limit autoimmune damage in the brain, it may also be detrimental by increasing risk of infection, and an immune balance must be maintained following AIS for ideal recovery[29]. As previously mentioned, post-stroke is characterized by lymphocytopenia; however, there have been no human studies that address the alterations of specific leukocyte subsets following AIS. In addition to total lymphocyte count, the overall function and lymphocyte activity following AIS. In one study in human AIS patients, while lymphocytopenia was observed following AIS, the remaining lymphocytes possessed normal functional activity[31]; however, there have been other studies that have shown a down-regulation of both CD3 and CD28, indicating decreased activation and function of peripheral $\mathrm{T}$ lymphocytes. Given the conflicting findings, the 
mechanisms mediating post-stroke immunosuppression remain unknown and warrant further investigation, but may offer a novel therapeutic strategy to reduce infection rate and increased secondary mortality in AIS patients.

\section{Inflammation and Risk of AIS}

In addition to the immune response following AIS, immune function and general inflammation can contribute to increased risk for AIS[32]. CV risk factors, such as hypertension, atherosclerosis, and obesity, are associated with chronic inflammation and immune dysregulation, which in addition to vascular dysfunction may contribute to their role in increased AIS risk[33]. Further, medications to reduce CV risk factors, such as anti-hypertensives or statins, are commonly prescribed following AIS and have been shown to improve AIS outcome. It is likely that these improvements in recovery may be due, in part, to reductions in inflammation or other beneficial effects on immune function attributed to remediation of AIS risk factors. Systemic infections, both bacterial and viral, occur in a large percentage of AIS within two weeks of AIS onset, indicating that infections may confer a higher risk of AIS[32]. Overall, alterations in immune function prior to AIS likely contribute to the immune response following AIS.

Clinical Trials Targeting the Immune Response in AIS

While several cell-based approaches and pharmacological therapeutics directly targeting the immune system have shown efficacy in reducing AIS severity in animal models of AIS, none of these approaches has shown efficacy in human clinical trials[34]. The most recently completed clinical trials targeting the immune response in AIS are summarized in Table 1.1. Given the differences between the immune systems of humans and animals[35], therapeutic interventions based on animal studies have failed to translate to 
the human population, and overcoming this translational gap will be crucial to targeting the immune system in AIS.

\section{Neutrophil-Lymphocyte Ratio in Ischemic Stroke}

The neutrophil-lymphocyte ratio (NLR) has been established as a marker of systemic inflammation and may also indicate the balance between innate and adaptive immune responses occurring systemically[36]. The NLR is calculated by dividing the total peripheral neutrophil count by the total peripheral leukocyte count. The NLR is currently used clinically in a wide variety of cancers to predict prognosis, tumor metastasis, and response to chemotherapy, and an elevated NLR is indicative of poor outcome on all measures[36]. While the NLR is not currently used in AIS clinical care, there is evidence to suggest that the NLR may be a useful prognostic biomarker of AIS severity and outcome. Specifically, our lab and others have shown that an NLR>5, independent of infarct volume, is associated with increased 60 day mortality [37, 38] and worse 90 day outcome, as assessed by the Modified Rankin Score (MRS)[39]. Further, in addition to the NLR, total neutrophil count was positively correlated with increased mortality, whereas total lymphocyte count was negatively correlated with mortality, suggesting a harmful role for neutrophils and a protective role of lymphocytes in AIS[38]. Further research is needed to determine the physiological mechanisms responsible for modulating the NLR following stroke; however, the NLR may be currently implemented in AIS clinical care and may offer additional insight into stratifying AIS patients into treatment groups or predicting outcome. 


\section{Arginase}

Arginase (ARG) is an enzyme that catalyzes the conversion of the semi-essential amino acid L-arginine to L-ornithine and urea (Figure 8) [40]. This reaction is the final step of the urea cycle to rid the body of excess nitrogen/ammonia through detoxification into urea[40]. ARG exists as two isoforms, encoded by distinct genes on separate chromosomes: ARG1 and ARG2[40]. ARG1 is the more abundant ARG isoform, located in the cytoplasm, and expressed primarily in the liver, and ARG2 is located within mitochondria mainly in the kidneys; however, both isoforms, particularly ARG1, are expressed by various cells/tissues, including endothelial cells, vascular smooth muscle cells, and leukocytes[41]. Constitutive expression of ARG, in both animals and humans, is summarized in Table 1.3. ARG gene expression can be stimulated by various stimuli, including pro-inflammatory cytokines, oxidized LDL cholesterol, glucose, angiotensin II, and hypoxia. ARG activity can also be increased in response to these stimuli, independent of changes in gene expression, and also by post-translational modifications, such as S-nitrosylation by $\mathrm{NO}[41]$.

\section{Arginase 1 and Vascular Function}

As mentioned previously, both endothelial and vascular smooth muscle cells express ARG1, suggesting a role for ARG1 in regulating vascular tone/function. L-arginine is a common substrate for both ARG1 and nitric oxide synthase (NOS)[41]. NOS is responsible for the production of NO, originally named "endothelium-derived relaxing factor". NO is a gaseous free radical, a signaling molecule that can freely diffuse across cellular membranes to elicit an action. As it's original name would suggest, NO plays a 
crucial role in vasodilation. Vascular endothelial cells use NO to signal to and induce relaxation in adjacent vascular smooth muscle cells, resulting in vasodilation and increased blood flow[41]. Because both vascular endothelial cells and smooth muscle cells express ARG1, excessive ARG1 can compete with NOS for L-arginine, resulting in impaired NO production and vascular dysfunction. Direct comparison of the $K_{m}$ values would suggest that the high $K_{m}$ of ARG1 (1-20 mM) compared to the $K_{m}$ of NOS (1-5 uM) should not allow for effective competition between these enzymes[42]. However, while the $K_{m}$ value of ARG1 is much higher than NOS, the rate of catalysis of ARG1 is much higher than NOS, thus when L-arginine concentrations are high enough to exceed the $K_{m}$ of ARG1, ARG1 activity exceeds NOS activity. ARG1 has been implicated in the "arginine paradox", a phenomenon where increased extracellular L-arginine is required to elicit NO production by endothelial NOS (eNOS), despite the fact that cytosolic concentrations of $\mathrm{L}$-arginine are well above the $\mathrm{K}_{\mathrm{m}}$ of eNOS. In addition to urea and L-ornithine, proline, and purines are also products of the L-arginine reaction, and these products promote both collagen synthesis and vascular smooth muscle cell hypertrophy, providing another mechanism by which ARG1 contributes to vascular dysfunction and arterial stiffness, independent of NOS[42].

Given the role of ARG1 in mediating vascular function, the role of ARG1 has been investigated in the development of CVD. Increased ARG1 expression and activity is associated with several CV risk factors, including hypertension, atherosclerosis, and diabetes, and ARG inhibition has been shown to improve CV function in both experimental and clinical studies (Table 1.3)[41]. 


\section{Vascular Function and Ischemic Stroke}

There are several non-invasive assessments used to measure the structure and function of vasculature in human subjects, including carotid-intima medial thickness (cIMT) and pulse wave velocity (PWV). IMT refers to the measurement of the thickness of the innermost layers of the arterial wall: the tunica intima and tunica media. Increased cIMT can result from arterial remodeling, such as increased extracellular matrix synthesis and vascular smooth muscle cell hypertrophy, and several CV risk factors, including atherosclerosis, diabetes, and hypertension are associated with increased cIMT[43]. Common carotid artery (CCA) IMT has been consistently identified as significant predictor of AIS risk[43]. CCA IMT values $\leq 0.87 \mathrm{~mm}$ are not associated with an increased risk of stroke; however, an increase in CCA IMT by $0.20 \mathrm{~mm}$ is associated with a 33 percent increase in AIS risk[44]. Further, CCA IMT appears to be most predictive of the largeartery AIS subtype, likely due to the strong association between cIMT and presence of atherosclerosis[45]. While cIMT provides a measure of vessel wall thickness, it is not a direct assessment of arterial stiffness; however, there is a strong correlation between cIMT and PWV. Carotid-femoral PWV (cfPWV) is considered to be a measure of aortic arterial stiffness, and increased cfPWV is associated with an increased risk of AIS[45]. Further, not only is cfPWV higher in AIS compared to control subjects, but cfPWV in AIS patients appears to be highest among lacunar AIS cases[45]. In addition, cfPWV is positively associated with several pro-inflammatory biomarkers, including C-reactive protein, TNFa, and IL-6[46]; however, it is unclear whether these inflammatory factors directly induce increases in cfPWV or indirectly through CV risk factors that are associated with an increase in cfPWV. Lastly, while cfPWV is considered a measure of 
aortic stiffness, brachial-ankle PWV (baPWV) is strongly correlated with the presence and degree of intracranial cerebral atherosclerosis[47], and thus may offer prognostic information in context of AIS.

\section{Arginase 1 and Immune Function}

In single-celled organisms, amino acid depletion, specifically L-arginine depletion, is used to inhibit the proliferation of other single-celled organisms competing for the same resources[48]. Similarly, it appears as metazoans utilize a similar strategy to limit undesired expansion of proliferating cells. ARG1 is constitutively expressed by both neutrophils and monocytes in humans and mice; however, while in mice ARG1 expression and activity can be induced by exposure to $\mathrm{TH}_{2}$ cytokines, human ARG1 expression and activity are not affected by these stimuli[48]. Circulating neutrophils and monocytes expressing ARG1 can deplete L-arginine both locally and systemically. During recent years, L-arginine depletion and ARG1 expression has been implicated as an immunosuppressive pathway in the human immune system. Specifically, both L-arginine depletion and ARG1 expression have been shown to inhibit T cell proliferation and inhibit T-cell responses to antigen[48]. The mechanisms by which L-arginine depletion/ARG1 may regulate T-cell function are summarized in Figure 1.9 [48]; however, these mechanisms have been described in-vitro and very little is known about the mechanisms of immunosuppression in-vivo. The most commonly described mechanism of ARG1induced immunosuppression is downregulation of the $\mathrm{CD} 3 \zeta$ chain of the $\mathrm{T}$ cell receptor. CD3 $\zeta$ is the main signal-transducing component of the $T$ cell receptor, and loss of this 
molecule results in a lack of T cell proliferation, activation, and in some cases, may induce apoptosis in these cells[48].

\section{Arginase 1 and Ischemic Stroke}

Given the relationship between ARG1 and the immune response, it is not surprising that ARG1 plays a role in AIS; however, there are only two experimental studies directly evaluating the role of ARG1 in an animal model of AIS. The first examined the changes in ARG1 localization in the rat brain before and at several time points following a photothrombotic stroke model[49]. Prior to stroke, ARG1 expression was localized to neurons only, however from 1 day to 30 days, there is a marked upregulation of ARG1 expression, not in neurons, but in inflammatory cells, such as microglia, macrophages, and astrocytes[49]. The second study, released in early 2015, described a mechanism of immunosuppression following murine MCAO mediated by ARG1 release from neutrophils[50]. The immunosuppression in this study was characterized by splenic atrophy, lymphocytopenia, and decreased T cell proliferation[50], which is identical to the clinical presentation of post-stroke immunosuppression in human AIS patients. To date, there have been no experimental studies evaluating the use of an ARG inhibitor, such as nor-NOHA, in improving outcomes and prevention of immunosuppression following MCAO.

In humans, there have been a limited number of studies that have examined the role of ARG1 following stroke. Two studies confirmed that ARG1 is the most significantly upregulated gene within 24 hours of AIS compared to control subjects[27, 28]. Further, ARG1 expression is highest in neutrophils[27], suggesting a potential role for neutrophil 
ARG1 in mediating AIS severity and immunosuppression; however, the role of ARG1 and the immune response following AIS in humans has not been characterized. 


\section{References}

1. Mozaffarian, D., et al., Heart disease and stroke statistics--2015 update: a report from the American Heart Association. Circulation, 2015. 131(4): p. e29-322.

2. Bushnell, C., et al., Guidelines for the prevention of stroke in women: a statement for healthcare professionals from the American Heart Association/American Stroke Association. Stroke, 2014. 45(5): p. 1545-88.

3. Ohira, T., et al., Risk factors for ischemic stroke subtypes: the Atherosclerosis Risk in Communities study. Stroke, 2006. 37(10): p. 2493-8.

4. Berg, A., et al., Poststroke depression: an 18-month follow-up. Stroke, 2003. 34(1): p. $138-43$.

5. Adams, H.P., Jr., et al., Classification of subtype of acute ischemic stroke. Definitions for use in a multicenter clinical trial. TOAST. Trial of Org 10172 in Acute Stroke Treatment. Stroke, 1993. 24(1): p. 35-41.

6. Petty, G.W., et al., Ischemic stroke subtypes: a population-based study of incidence and risk factors. Stroke, 1999. 30(12): p. 2513-6.

7. Amarenco, P., et al., Classification of stroke subtypes. Cerebrovasc Dis, 2009. 27(5): p. 493-501.

8. Christou, I., et al., Arterial status after intravenous TPA therapy for ischaemic stroke. A need for further interventions. Int Angiol, 2001. 20(3): p. 208-13.

9. Fransen, P.S., et al., MR CLEAN, a multicenter randomized clinical trial of endovascular treatment for acute ischemic stroke in the Netherlands: study protocol for a randomized controlled trial. Trials, 2014. 15: p. 343.

10. Donnan, G.A., The impact of MR CLEAN. Int J Stroke, 2015. 10(2): p. 139. 
11. Netter, F., Atlas of Human Anatomy. 6th ed. 2014, USA: Saunders.

12. Siegel, G.J., et al., Basic Neurochemistry. 1999.

13. Deb, P., S. Sharma, and K.M. Hassan, Pathophysiologic mechanisms of acute ischemic stroke: An overview with emphasis on therapeutic significance beyond thrombolysis. Pathophysiology, 2010. 17(3): p. 197-218.

14. Easton, A.S., Neutrophils and stroke - can neutrophils mitigate disease in the central nervous system? Int Immunopharmacol, 2013. 17(4): p. 1218-25.

15. Hock, B.D., et al., Effect of activated human polymorphonuclear leucocytes on $T$ lymphocyte proliferation and viability. Immunology, 2012. 137(3): p. 249-58.

16. Grogaard, B., et al., Delayed hypoperfusion after incomplete forebrain ischemia in the rat. The role of polymorphonuclear leukocytes. J Cereb Blood Flow Metab, 1989. 9(4): p. 500-5.

17. Use of anti-ICAM-1 therapy in ischemic stroke: results of the Enlimomab Acute Stroke Trial. Neurology, 2001. 57(8): p. 1428-34.

18. Gu, L., et al., T Cells and Cerebral Ischemic Stroke. Neurochem Res, 2015. 40(9): p. $1786-91$.

19. Xiong, X., et al., Increased Brain Injury and Worsened Neurological Outcome in Interleukin-4 Knockout Mice After Transient Focal Cerebral Ischemia. 2011.

20. Neuroprotection by IL-10-producing MOG CD4+ T cells following ischemic stroke. 2005. 233(Issues 1-2): p. 125-132.

21. Li, G.Z., et al., Expression of Interleukin - 17 in Ischemic Brain Tissue. Scandinavian Journal of Immunology, 2015. 62(5): p. 481-486. 
22. Gelderblom, M., et al., Neutralization of the IL-17 axis diminishes neutrophil invasion and protects from ischemic stroke. Blood, 2012. 120(18): p. 3793-802.

23. Brait, V.H., et al., Importance of T lymphocytes in brain injury, immunodeficiency, and recovery after cerebral ischemia. Journal of Cerebral Blood Flow \& Metabolism, 2012. 32(4): p. 598-611.

24. $\mathrm{Li}, \mathrm{P}$., et al., Adoptive regulatory $\mathrm{T}$-cell therapy protects against cerebral ischemia. Ann Neurol, 2013. 74(3): p. 458-71.

25. Kleinschnitz, C., et al., Regulatory $T$ cells are strong promoters of acute ischemic stroke in mice by inducing dysfunction of the cerebral microvasculature. Blood, 2013. 121(4): p. 679-91.

26. Jickling, G.C. and F.R. Sharp, Blood biomarkers of ischemic stroke. Neurotherapeutics, 2011. 8(3): p. 349-60.

27. Tang, Y., et al., Gene expression in blood changes rapidly in neutrophils and monocytes after ischemic stroke in humans: a microarray study. J Cereb Blood Flow Metab, 2006. 26(8): p. 1089-102.

28. Barr, T.L., et al., Genomic biomarkers and cellular pathways of ischemic stroke by RNA gene expression profiling. Neurology, 2010. 75(11): p. 1009-14.

29. Dirnagl, U., et al., Stroke-Induced Immunodepression. 2007.

30. Emsley, H.C. and S.J. Hopkins, Post-stroke immunodepression and infection: an emerging concept. Infect Disord Drug Targets, 2010. 10(2): p. 91-7.

31. Vogelgesang, A., et al., Functional Status of Peripheral Blood T-Cells in Ischemic Stroke Patients, in PLoS One. 2010. 
32. Lindsberg, P.J. and A.J. Grau, Inflammation and Infections as Risk Factors for Ischemic Stroke. 2003.

33. Willerson, J.T. and P.M. Ridker, Inflammation as a Cardiovascular Risk Factor. 2004.

34. Durukan, A. and T. Tatlisumak, Acute ischemic stroke: overview of major experimental rodent models, pathophysiology, and therapy of focal cerebral ischemia. Pharmacol Biochem Behav, 2007. 87(1): p. 179-97.

35. Mestas, J. and C.C.W. Hughes, Of Mice and Not Men: Differences between Mouse and Human Immunology. 2004.

36. Guthrie, G.J., et al., The systemic inflammation-based neutrophil-lymphocyte ratio: experience in patients with cancer. Crit Rev Oncol Hematol, 2013. 88(1): p. 21830.

37. Tokgoz, S., et al., Neutrophil lymphocyte ratio as a predictor of stroke. J Stroke Cerebrovasc Dis, 2013. 22(7): p. 1169-74.

38. Tokgoz, S., et al., Is neutrophil/lymphocyte ratio predict to short-term mortality in acute cerebral infarct independently from infarct volume? J Stroke Cerebrovasc Dis, 2014. 23(8): p. 2163-8.

39. Brooks, S.D., et al., Admission neutrophil-lymphocyte ratio predicts 90 day outcome after endovascular stroke therapy. J Neurointerv Surg, 2014. 6(8): p. 57883.

40. Caldwell, R.B., et al., Arginase: an old enzyme with new tricks. Trends Pharmacol Sci, 2015. 36(6): p. 395-405. 
41. Pernow, J. and C. Jung, Arginase as a potential target in the treatment of cardiovascular disease: reversal of arginine steal? Cardiovasc Res, 2013. 98(3): p. 334-43.

42. Santhanam, L., et al., Arginase and vascular aging. J Appl Physiol (1985), 2008. 105(5): p. 1632-42.

43. Mattace-Raso, F.U.S., et al., Arterial Stiffness and Risk of Coronary Heart Disease and Stroke. 2006.

44. Carotid-Artery Intima and Media Thickness as a Risk Factor for Myocardial Infarction and Stroke in Older Adults - NEJM. 2015.

45. Arterial stiffness indexes in acute ischemic stroke: Relationship with stroke subtype. 2010. 211(1): p. 187-194.

46. Immune-inflammatory markers and arterial stiffness indexes in subjects with acute ischemic stroke. 2010. 213(1): p. 311-318.

47. Kim, J., et al., The association between cerebral atherosclerosis and arterial stiffness in acute ischemic stroke. Atherosclerosis, 2011. 219(2): p. 887-91.

48. Bronte, V. and P. Zanovello, Regulation of immune responses by L-arginine metabolism. Nat Rev Immunol, 2005. 5(8): p. 641-54.

49. Quirie, A., et al., Effect of stroke on arginase expression and localization in the rat brain. Eur J Neurosci, 2013. 37(7): p. 1193-202.

50. Sippel, T.R., et al., Arginase I release from activated neutrophils induces peripheral immunosuppression in a murine model of stroke. J Cereb Blood Flow Metab, 2015. 35(10): p. 1657-63. 


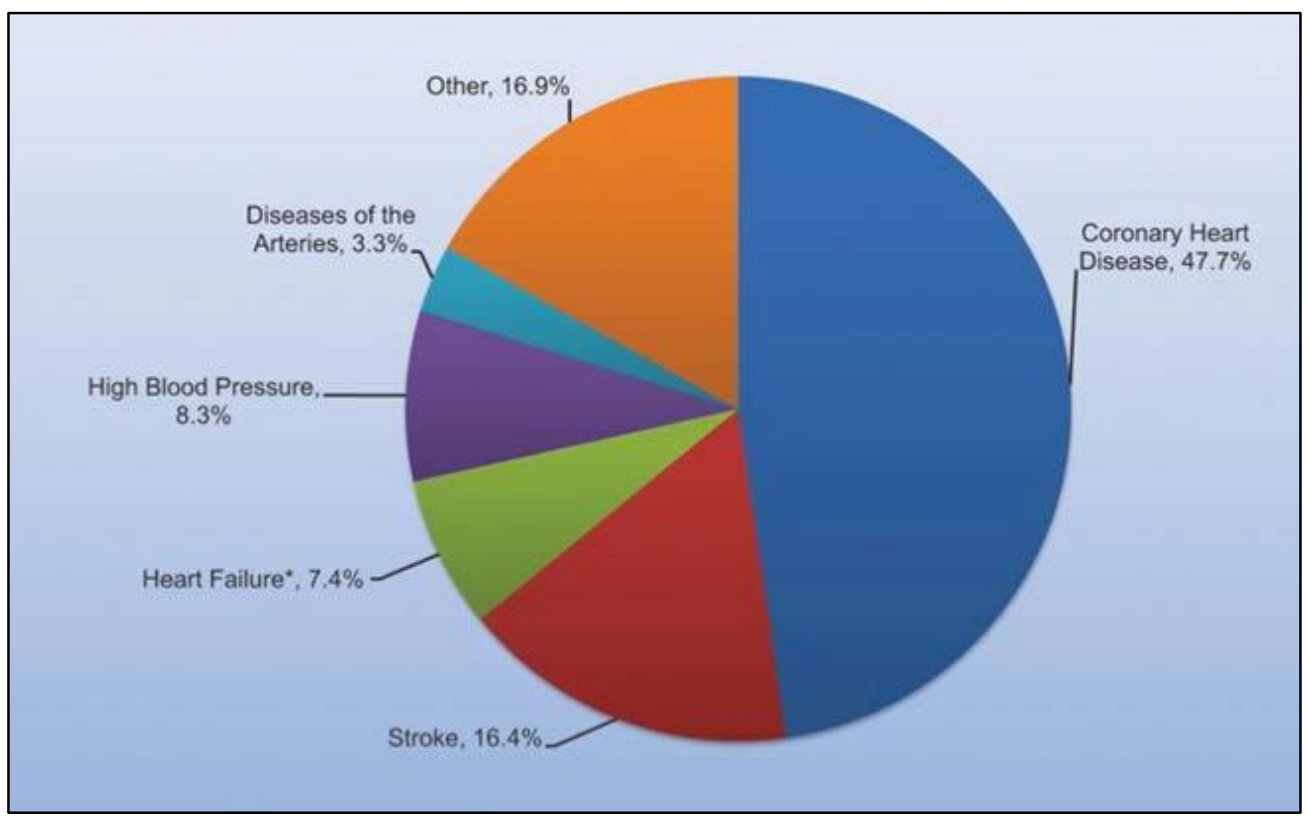

Figure 1.1: Percentage Breakdown of Deaths Attributable to Cardiovascular Disease (United States 2011). Taken from Mozzafarian et al. 2015. Circulation. 


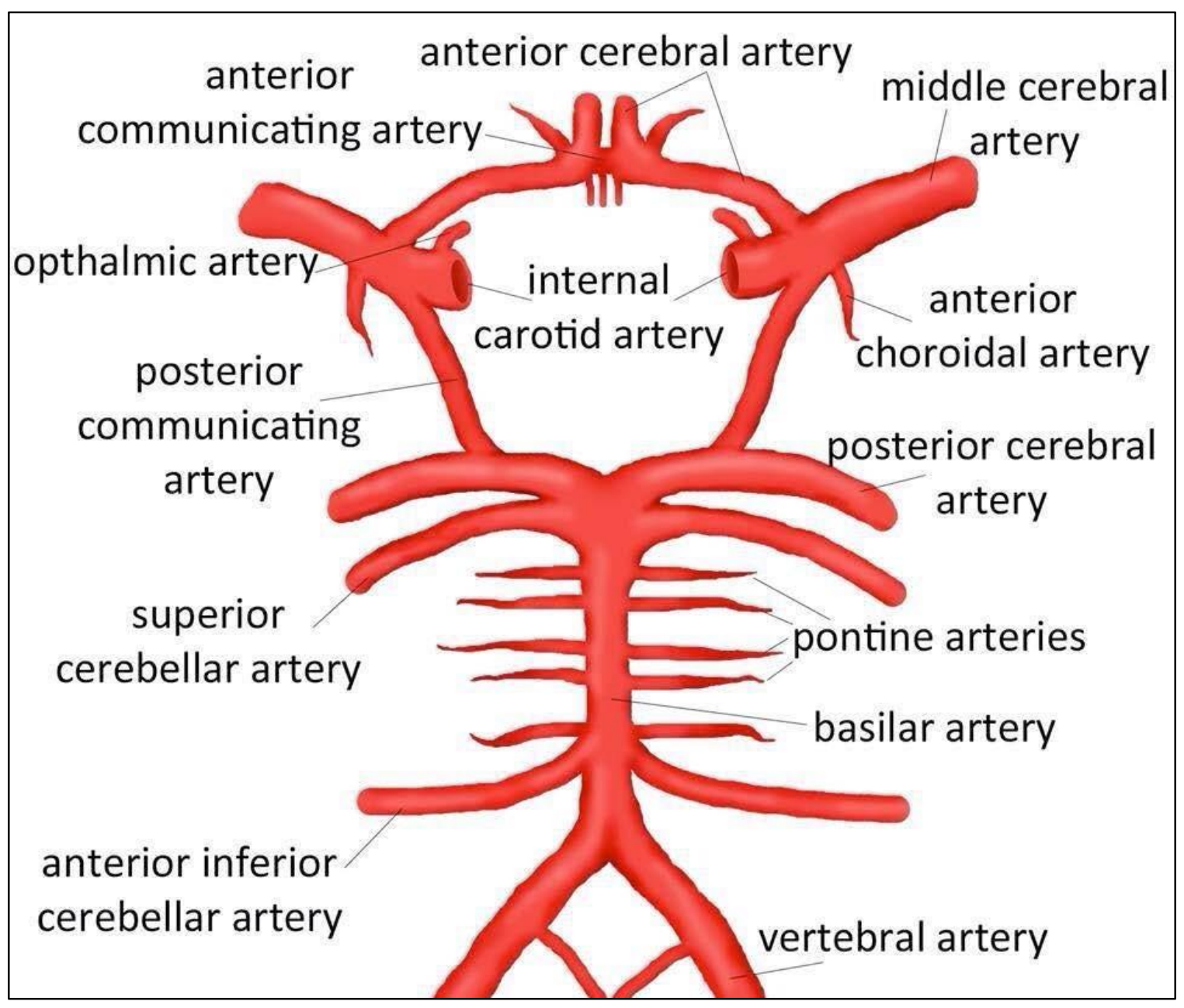

Figure 1.2: Circle of Willis: Cerebral Vasculature Anatomy. Taken from http://www.chw.org/medical-care/birthmarks-and-vascular-anomaliescenter/conditions/phace-syndrome/phace-syndrome-handbook/abnormalities-of-thehead-and-neck-arteries/ 


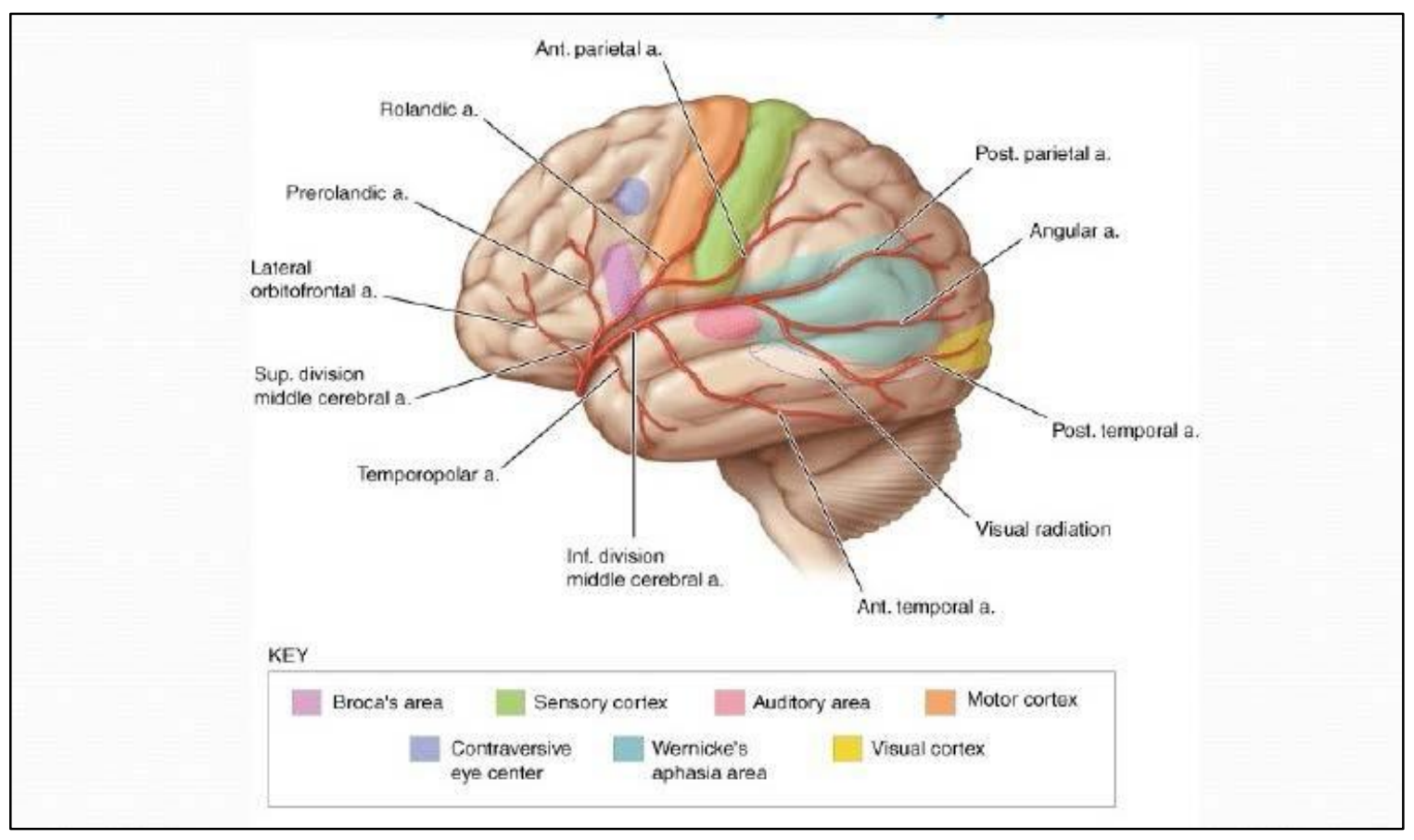

Figure 1.3: Cerebral Territory Supplied by the Middle Cerebral Artery. Taken from https://www.studyblue.com/notes/note/n/aced-neuro-/deck/11853080 . 


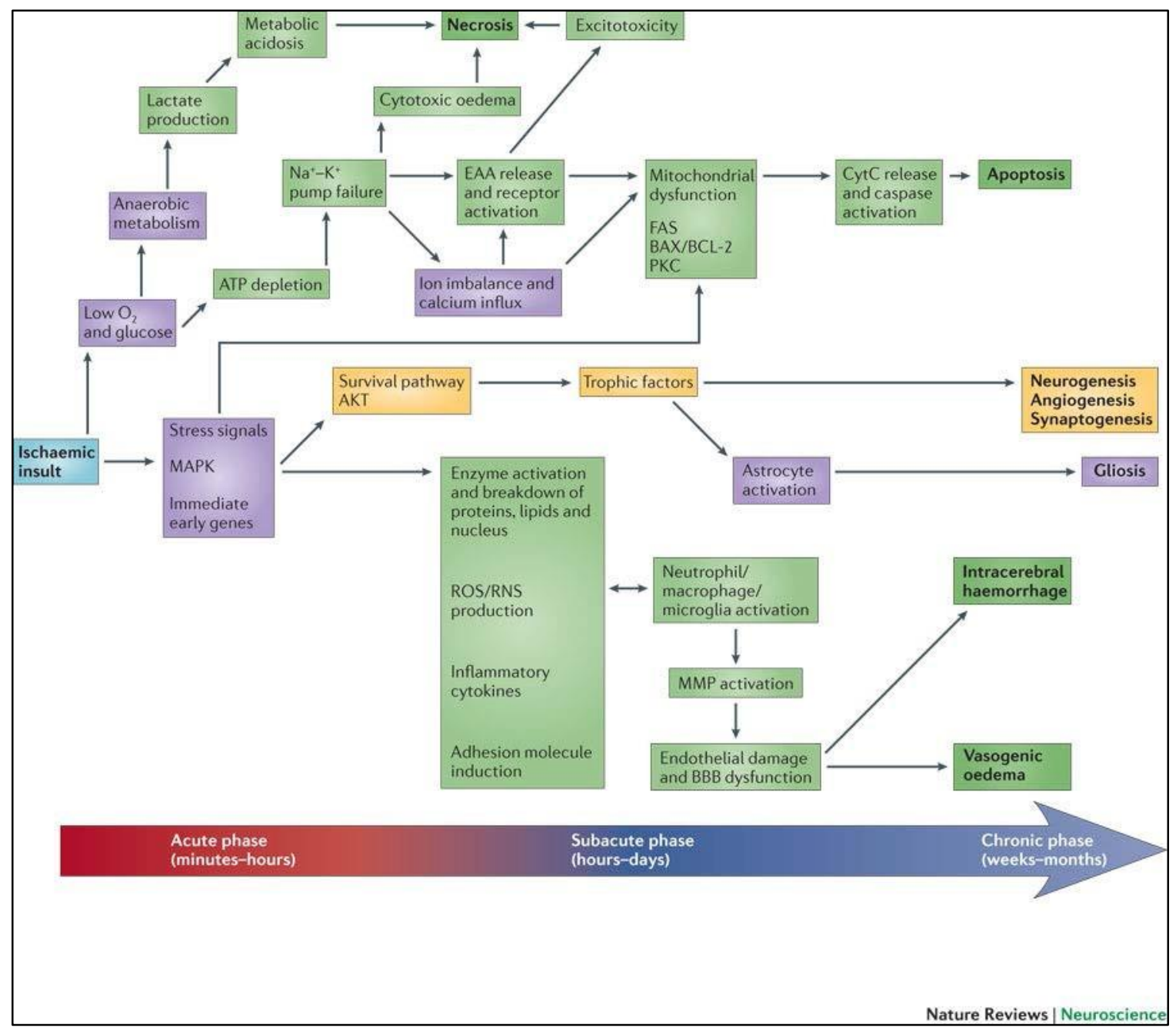

Figure 1.4: Ischemic Cascade Following Ischemic Stroke. Taken from Yenari and Han 2012. Nature Reviews Neuroscience. 


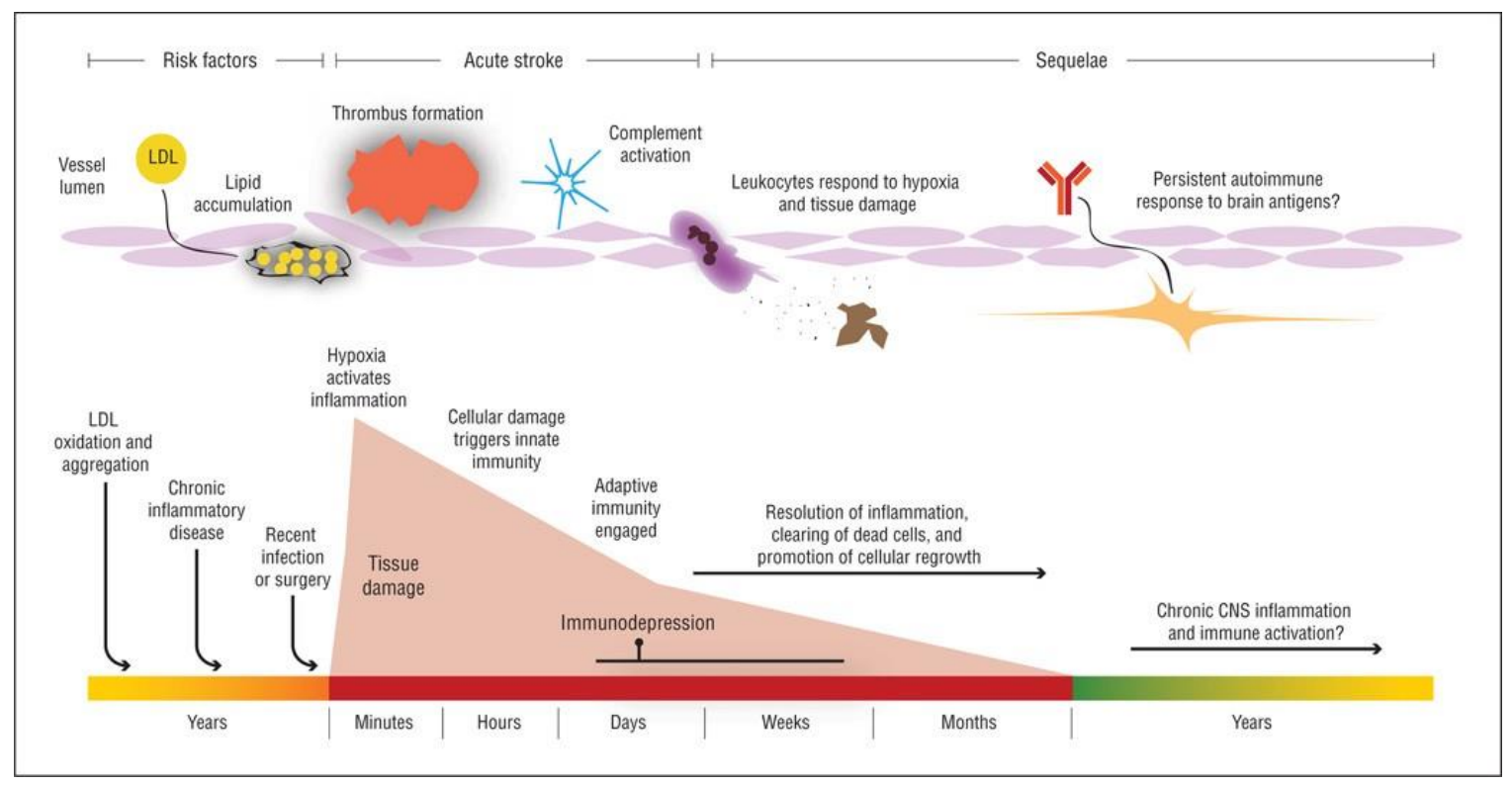

Figure 1.5: Immune Response to Ischemic Stroke. Taken from Kamel and ladecola 2012. Archives of Neurology. 


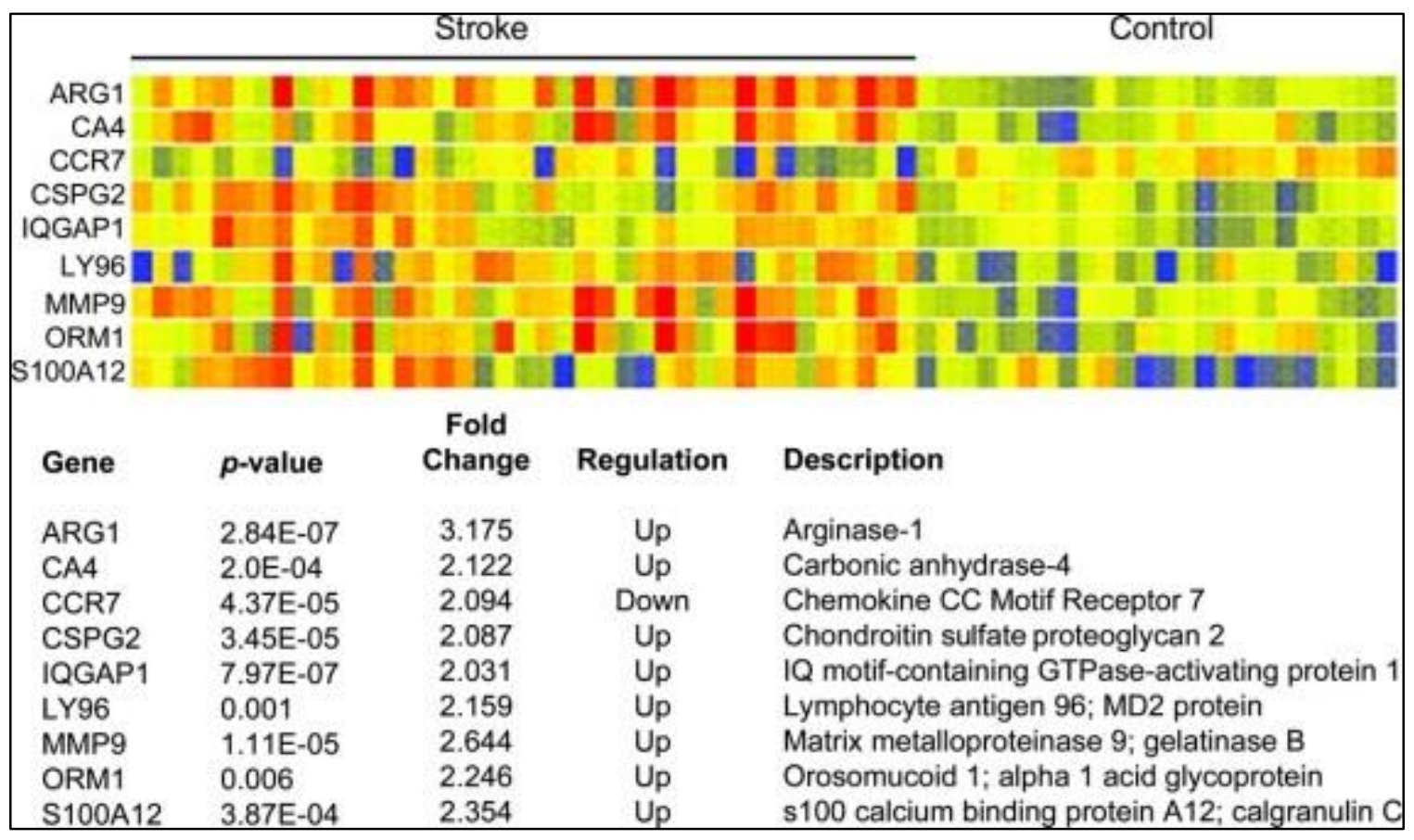

Figure 1.6: Immune-Related Genes Altered Within 24 Hours of Ischemic Stroke Compared to Control Subjects. Taken from Barr et al. 2010. Neurology. 


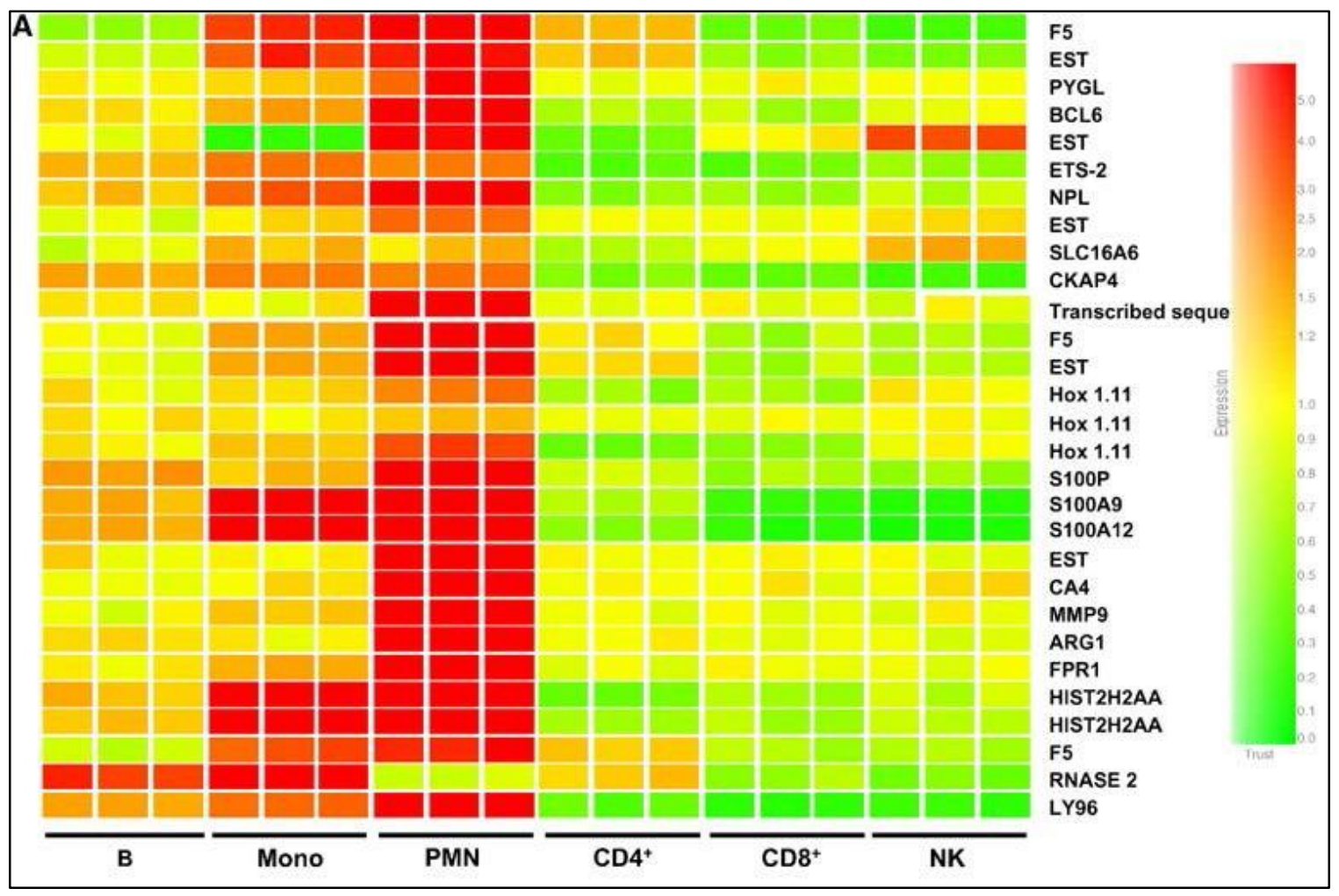

Figure 1.7: Leukocyte-Specific Expression of Genes Altered Within 24 Hours of Ischemic Stroke in Control Subjects. Taken from Tang et al. 2006. Journal of Cerebral Blood Flow and Metabolism. 


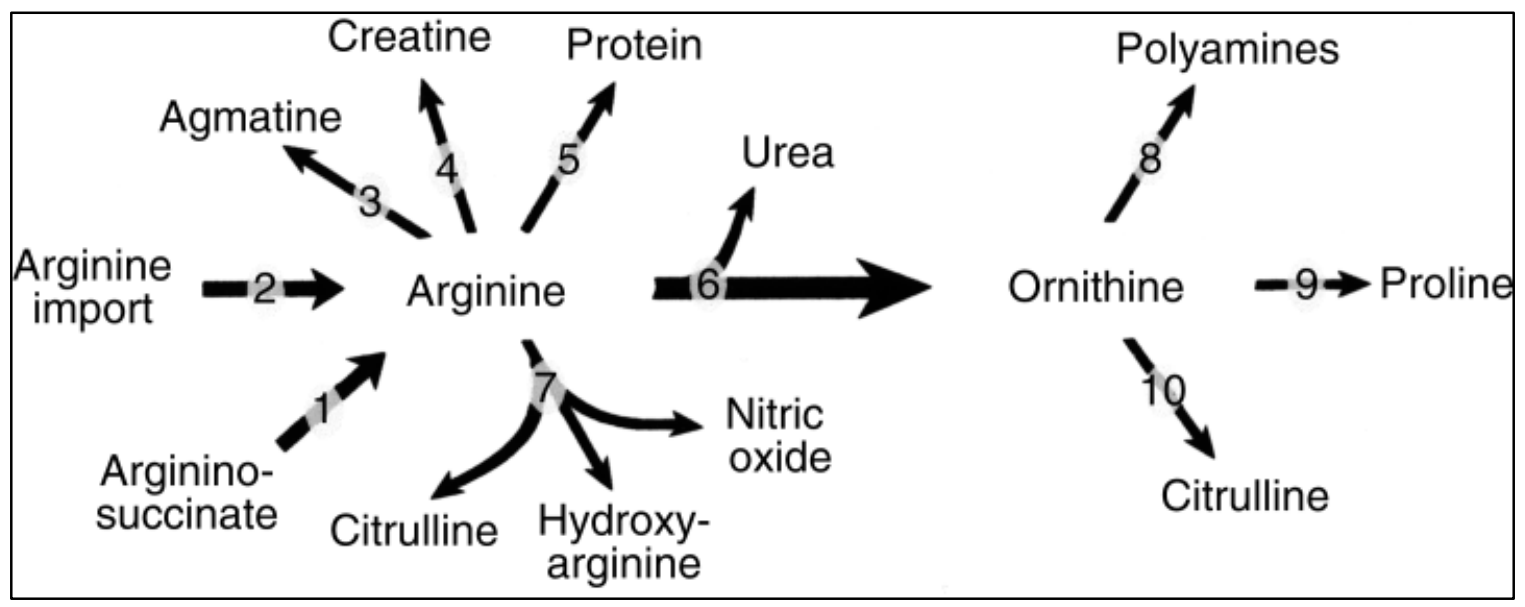

Figure 1.8: Chronological Order of L-Arginine Metabolism by Arginase and Products. Taken from Waddington 2002. Kidney International. 


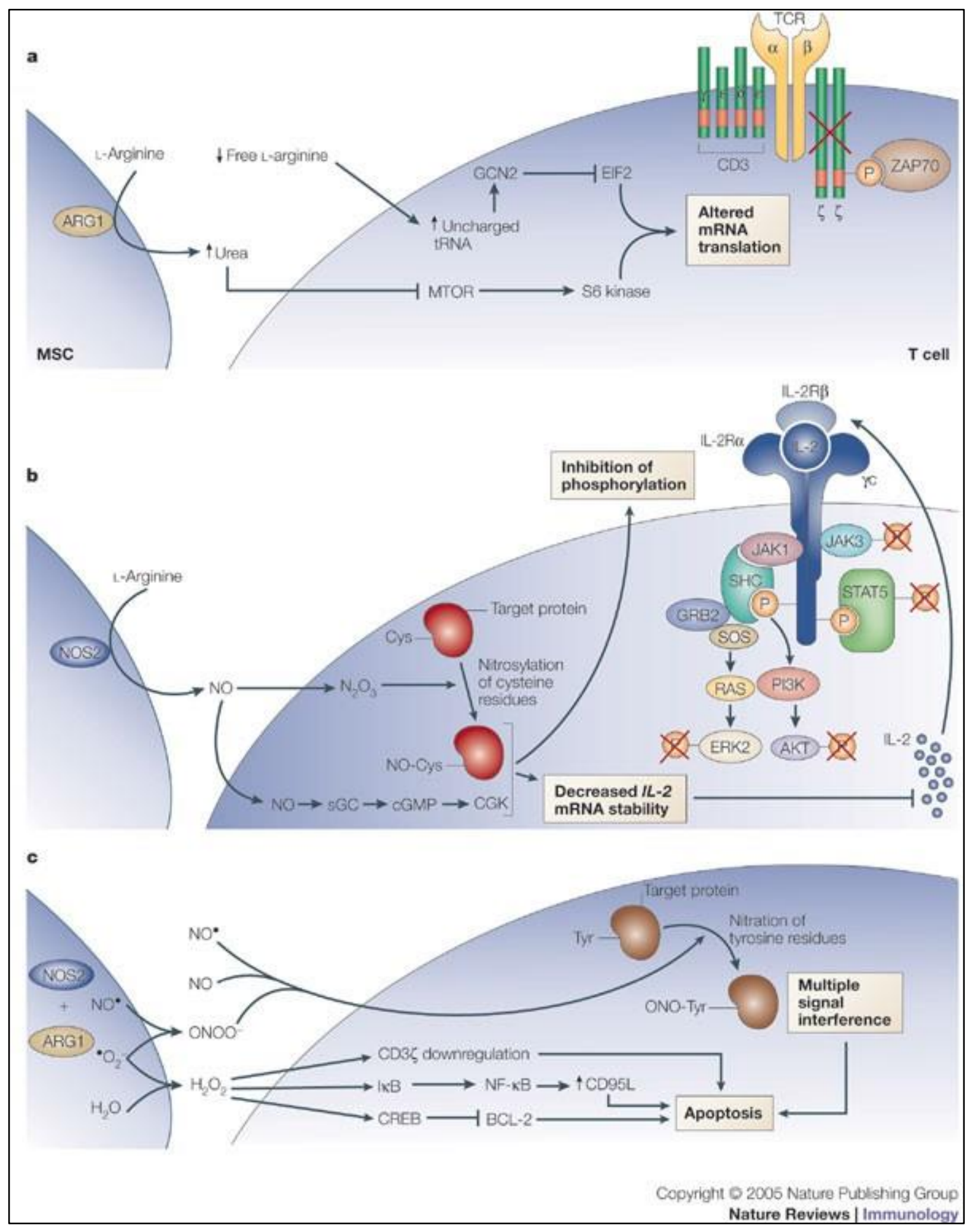

Figure 1.9: Potential Pathways Controlling the T Cell Response to L-Arginine Metabolism. Taken from Bronte and Zanovello 2005. Nature Reviews Immunology. 


\begin{tabular}{ccc}
\hline Mechanism and Compound & Trial & Result \\
\hline $\begin{array}{c}\text { Leukocyte Adhesion Inhibition } \\
\text { Anti-ICAM1 antibody } \\
\text { HU23F2G }\end{array}$ & EAST & Adverse Effect \\
Neutrophil Modulation & HALT & Nonefit \\
$\begin{array}{c}\text { Neutrophil Inhibitory Factor } \\
\text { Cytokine Inhibition }\end{array}$ & Phase II & No Benefit \\
IL-1 Receptor Antagonist & Phase II & No Benefit \\
& & \\
\hline
\end{tabular}

Table 1.1: Clinical Trials Targeting the Immune Response in Ischemic Stroke. Adopted from Durukan et al. 2007. Pharmacology Biochemistry and Behavior. 
Table 2 Interventional studies with arginase inhibitors in experimental and clinical studies

\begin{tabular}{|c|c|c|c|}
\hline Model patients & Intervention & Main effect & Reference \\
\hline \multicolumn{4}{|l|}{ Atherosclerosis/coromry artery disease } \\
\hline \multirow[t]{2}{*}{ ApoE ${ }^{-t /}$ mice } & Gronic intesion of BEC by osmotic mnipurnp & $\begin{array}{l}\text { Improvernent in endothelal function. } \\
\text { reduction in superoxide production, } \\
\text { reduction in atherosclerosis }\end{array}$ & 31 \\
\hline & Gronic ord $\mathrm{ABH}$ administration & & \\
\hline Patients with coronury artery disense & ta intusion of nor-NOHA & Improvernest in forearm endothelial function. & 70 \\
\hline \multicolumn{4}{|l|}{ Myocardal VR } \\
\hline Rat in vio & W nor-NOHA before ischemia & Reduction in infart size & se \\
\hline \multirow[t]{2}{*}{ Pig in vio } & Ic nor-NOHA before reperfusion & Reduction in infarct sze & si \\
\hline & DFMO and non-NOHA ex vio & Improvernest in endotheifal function & 49 \\
\hline Mouse exvio & NonNOHA ex vioo & Improvernent in endothelial function & $\$ 2$ \\
\hline \multicolumn{4}{|l|}{ Diabetes melitis } \\
\hline Rat type 1 dabetes & DFMO ex vio & Improvernest in endothelial function & $n$ \\
\hline \multirow[t]{2}{*}{ Moxse type 1 dabetes } & BEC ex vivo & Improvernest in endothelal function & 62 \\
\hline & $\mathrm{ABH} s \mathrm{f}$ for 5 days & Improvernent in retrial endotheival function & sa \\
\hline Rat type 2 dabetes & NonNOHA iv & Inproved myocardal microvascular function & 4 \\
\hline Patients with type 1 and type 2 diabetes & NonNOHA ex wo & $\begin{array}{l}\text { Improvernest in corociary arteriolar } \\
\text { endotheial function }\end{array}$ & 49 \\
\hline $\begin{array}{l}\text { Patients with type } 2 \text { dabetes and coronary } \\
\text { artery disease }\end{array}$ & ta infusion of nor- $\mathrm{NOHA}$ & Improvernest in forearm endothelial function. & 70 \\
\hline \multicolumn{4}{|l|}{ Heart falure } \\
\hline Patients with heart falure & Topica application of nor NOHA & Incresse in subingal microvascuar flow & $7 a$ \\
\hline \multicolumn{4}{|l|}{ Hypertersion } \\
\hline Pigs (aortic coartatation) & NonNOHA ex vio & Improvernent in endothelial function & as \\
\hline Spontaneously hypertensive rats & Non-NOHA ip for 3 weels & $\begin{array}{l}\text { Reduction in MAP, reduction in myogenic } \\
\text { torie, improvernert in endothelial function }\end{array}$ & as \\
\hline Patients with hypertersion & $\mathrm{BEC}+$ nor-NOHA via cutaneous microdials is & Increised reflex cutaneous vasodiatation & at \\
\hline \multicolumn{4}{|l|}{ РАH } \\
\hline Rats with pulmorary embolism & Wnor-NOHA & $\begin{array}{l}\text { Reduced the rise in puimorary vascular } \\
\text { resistance in vio and improved endothelial } \\
\text { function ex vivo }\end{array}$ & 9194 \\
\hline \multicolumn{4}{|l|}{ High age } \\
\hline Rats & $B E C$ ex vio & Improved endothelial function & 21 \\
\hline Humars: & BEC + non - NOHA va cutaneous microdiajs is & Increased reflex cutaneo us vasodiatation & ton \\
\hline
\end{tabular}

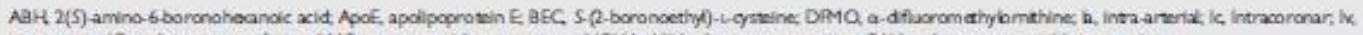

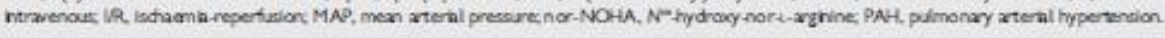

Table 1.2: Interventional Studies with Arginase Inhibitors in Experimental and Clinical Studies. Taken from Pernow and Jung 2013. Cardiovascular Research. 


\section{Chapter 2}

The Role of Arginase 1 in Post-Stroke Immunosuppression and Ischemic Stroke Severity

Ashley B. Petrone, BS 1,2, Grant C. O'Connell, MS 2,3, Michael D. Regier, PhD ${ }^{2,4}$, Paul D. Chantler ${ }^{5,6}$ James W. Simpkins, PhD ${ }^{2,7}$, and Taura L. Barr, $\mathrm{PhD}{ }^{2,8}$

${ }^{1}$ Dept. of Neurobiology and Anatomy, ${ }^{2}$ Center for Basic and Translational Stroke Research, ${ }^{3}$ Dept. of Pharmaceutical Sciences, ${ }^{4}$ Dept. of

Biostatistics, ${ }^{5}$ Dept. of Exercise Physiology, ${ }^{6}$ Center for Cardiovascular and Respiratory Sciences, ${ }^{7}$ Dept. of Physiology and Pharmacology, ${ }^{8}$ School of Nursing West Virginia University, One Medical Center Drive, Morgantown WV 26506 
Background: A balanced immune system response plays an important role in acute ischemic stroke (AIS) recovery. Our laboratory has previously identified several immunerelated genes, including Arginase 1 (ARG1), with altered expression in human AIS patients. The neutrophil-lymphocyte ratio (NLR) may be a marker of the degree of immune dysregulation following AIS; however, the molecular mechanisms that may mediate the NLR are unknown. The purpose of this study was to: (1) Examine the relationship between ARG1, NLR, and AIS severity and (2) To Utilize Principal Component Analysis (PCA) to statistically model multiple gene expression changes following AIS.

Methods: AIS patients and stroke-free control subjects were recruited and blood samples were collected from AIS patients within 24 hours of stroke symptom onset. White blood cell differentials were obtained at this time to calculate the NLR. Gene expression was measured using real-time PCR. Principal component analysis (PCA) with varimax rotation was used to develop composite variables consisting of a five-gene profile.

Results: ARG1 was positively correlated with NLR ( $r=0.57, p=0.003)$, neutrophil count $(r=0.526, p=0.007)$, NIHSS $(r=0.607, p=0.001)$ and infarct volume $(r=0.27, p=0.051)$. PCA identified three principal components that explain $84.4 \%$ of variation in the original patient gene dataset comprised of ARG1, LY96, MMP9, s100a12, and PC1 was a significant explanatory variable for NIHSS $(p<0.001)$ and NLR $(p=0.005)$.

Conclusions: Our study suggests a novel relationship between ARG1, NLR and stroke severity, and the NLR is an under-utilized clinically available biomarker to monitor the post-stroke immune response. 


\section{Introduction}

A balanced immune system response plays an important role in acute ischemic stroke (AIS) recovery. [1, 2] AIS results in a well described immediate, but non-specific innate inflammatory response. Innate inflammatory processes activate adaptive immune cells responsible for controlling inflammation, modulating ischemic damage and promoting neurogenesis.[3] In some patients, for reasons poorly understood, the adaptive immune system is incapable of responding to the innate inflammatory signals, resulting in poststroke immune suppression. Stroke-induced adaptive immune suppression is associated with greater stroke severity, increased risk of infection and poor recovery, and is characterized by decreased $\mathrm{T}$ lymphocyte function, and peripheral cell counts and increased inflammatory cytokines. [1, 4] Human stroke genomic biomarker studies have identified a common panel of five genes responding to AIS in the peripheral blood: Arginase 1 (ARG1), lymphocyte antigen 96 (LY96), matrix metalloproteinase 9 (MMP9), s100 calcium binding protein A12 (s100A12), and chemokine CC motif receptor 7 (CCR7).[5, 6] Our laboratory has also shown that the neutrophil-lymphocyte ratio (NLR) is a simple, inexpensive biomarker that may be used to predict outcome following AIS and may be a clinically available marker of the degree of immune dysregulation following AIS[7, 8]; however, the relationship between the NLR and these genomic biomarkers following AIS is unknown.

$A R G 1$ is consistently upregulated in the whole blood of AIS patients.[5, 6] The traditional role for the enzyme ARG1 is catalyzing the metabolism of the substrate Larginine to L-ornithine and urea.[9] Because ARG1 and nitric oxide synthase (NOS) share L-arginine as a common substrate, ARG1 can be viewed as a competitive inhibitor of 
NOS. In addition to this traditional role, ARG1, derived from innate immune cells, has been shown to mediate the adaptive immune response.[10] In humans, ARG1 expression is highest in neutrophils compared to other peripheral blood leukocytes,[5] and ARG1 protein released from neutrophils suppresses $\mathrm{T}$ lymphocyte proliferation through downregulation of $T$ lymphocyte $\mathrm{CD} 3 \zeta$ chain.[10] Until very recently, ARG1-induced lymphocyte suppression had not been shown in the context of AIS. Sippel et al. reported that ARG1protein released from neutrophils induces lymphopenia in a murine model of stroke.[11] Given the differences between murine and human immune systems, [12] it remains to be determined whether ARG1 induces immunosuppression in human AIS patients.

The purpose of this pilot study was to: (1) Examine the relationship between ARG1, NLR, and AIS severity. We hypothesized that increased ARG1 expression and serum protein activity is associated with an increased NLR, giving rise to increased AIS severity and poor outcome. (2) To validate the role of ARG1 as a novel biomarker of immune suppression in AIS, and (3) To Utilize Principal Component Analysis (PCA) to statistically model multiple gene expression changes following AIS.

\section{Materials and Methods}

\section{Subject Recruitment}

Informed consent was obtained from all individual participants included in the study. AIS patients and stroke-free control subjects were recruited from Ruby Memorial Hospital (Morgantown, WV). Male and female AIS patients were eligible for recruitment if the

following inclusion criteria were met: (1) age $\geq 18$ years, (2) confirmation of acute stroke 
by neuroimaging (CT or MRI), and (3) had blood drawn within 24 hours of symptom onset/"last-known normal", prior to thrombolysis or interventional treatment. Patient data from the medical record were reviewed and recorded, including (1) National Institutes of Health Stroke Scale (NIHSS), (2) clinical laboratory analyses, including white blood cell differential, and (3) brain imaging (CT/MRI). Stroke-free control subjects were eligible for recruitment if the following inclusion criteria were met: (1) age $\geq 18$ years, and (2) no history of AIS, transient ischemic attack, brain injury, or other overt central nervous system disease, and (3) recent hospitalization. Medical histories were obtained directly from stroke-free control subjects; however, complete access to medical records of control subjects was not available.

\section{Infarct Volume Calculation}

Brainlab iPlan® software was used to manually trace and calculate infarct volume from either CT or MRI images. All images were obtained within 24 hours of symptom onset, regardless of modality. All infarct volume calculations were verified by a neuroradiologist at Ruby Memorial Hospital (Morgantown, WV).

\section{Research Protocol Approval and Informed Consent}

This study received approval for human subject research from the institutional review boards of West Virginia University and Ruby Memorial Hospital (Morgantown, WV). Written informed consent was obtained from all subjects or their authorized representatives prior to performing study procedures. 


\section{Blood Collection}

Peripheral venous whole blood was drawn from stroke subjects no later than 24 hours of stroke symptom onset. Blood was collected into PAXgene文 Blood RNA tubes (BectonDickinson). Immediately after blood collection, tubes were inverted 8-10 times and stored at $-80^{\circ} \mathrm{C}$ until analysis. Whole blood was collected in serum separator tubes, centrifuged at $4000 \mathrm{~g}$, aliquoted into microcentrifuge tubes within one hour of collection, and stored at $-80^{\circ} \mathrm{C}$ until analysis.

\section{RNA Extraction}

PAXgene女 Blood RNA tubes were thawed overnight (16-20 hrs) at room temperature prior to RNA extraction. The PAXgene Blood RNA kit (Pre-Analytix) was used to purify/extract intracellular RNA, per manufacturer's instructions. RNA concentration and quality was determined by absorbance using a Take3 Trio Microplate (BioTek夕) read on a Syntek Hybrid Plate Reader, and analyzed using Gen5 (BioTek) software. A260/A280 values between 1.8 and 2.2 were considered acceptable RNA quality.

\section{Gene Expression Analysis}

RNA was converted to cDNA using the High-Capacity Reverse Transcription Kit (Applied Biosystems). cDNA (10 ng) was used for quantitative real-time PCR amplification using SYBR Green chemistry using the Rotor-Gene Q real-time PCR cycler (Qiagen). The following Quantitect primers (Qiagen) were used: ARG1 (NM_000045, NM_001244438), LY96 (NM_015364), MMP9 (NM_004994), s100a12 (NM_005621), and CCR7 (NM_001838). Gene expression was normalized using both PPIB (NM_000942) and B2M 
(NM_004048). These reference genes are known to be stably expressed in whole blood of ischemic stroke patients.[13] We confirmed that both PPIB and B2M were stably expressed across our subject population $(S D<1)$ (unpublished data). Fold change differences were calculated by the $\Delta \Delta \mathrm{CT}$ method. ${ }^{[14]}$

\section{Measurement of Serum ARG1 Protein Activity}

Serum ARG1 protein activity levels were determined in AIS patients using an Arginase Activity Assay Kit (Sigma-Aldrich). All procedures were performed per manufacturer's instructions. Activity data is presented in units/L.

\section{Sample Size Estimation}

Post-hoc power analyses were conducted to determine achieved power, given alpha level, sample size, and effect size using G*Power3.[15] We performed two independent power analyses for the two primary aims of the study: (1) To evaluate the relationship of ARG1 and stroke severity, as measured by NIHSS, and (2) To compare ARG1 expression between stroke and control. Using a linear regression model, controlling for seven predictors (age, sex, smoking, hypertension, hyperlipidemia, diabetes and prior stroke), we determined that our sample size of $n=26$ and effect size 0.5 was sufficient to detect differences in ARG1 expression along the $\mathrm{NIH}$ stroke severity score scale with $88 \%$ power at an alpha level of 0.05 . Using a linear regression model, controlling for eight predictors (case (control v. stroke), age, sex, smoking, hypertension, hyperlipidemia, diabetes, and prior stroke), we determined that our sample size $n=45$ and effect size 0.5 was sufficient 
to detect differences in ARG1 expression between stroke and controls with 99\% power at an alpha level of 0.05 .

\section{Statistical Analysis}

The Mann-Whitney $U$ test was used for testing differences between cases and controls for continuous variables. The Fisher's exact test was used to compare cases and control for categorical variables. Spearman's correlation, using the transformed variables, with a continuity correction was used for continuous variable bivariate relationships.

If the variable's skew was greater than 0.5 the variable was transformed using the Box-Cox Transformation (Box \& Cox, 1964). If there were negative values, we used the exponential transformation (Manly, 1976). The application of the Box-Cox and exponential transformations provided substantial reductions in variable skew and hence mitigated the presence of variable specific outliers. All variables were scaled by their standard deviation prior to applying any transformation.

Principal component analysis (PCA) with varimax rotation was used to develop composite variables consisting of a five-gene profile. The relevant components were chosen such that at least $80 \%$ of the original variation in the data was explained. Following the identification of the principal components, principal component regression was performed to assess the relationship between the derived components and the outcomes of interest.

Linear regression model selection used Akaike information criterion (AIC) backwards, stepwise selection to identify informative variables from among the set of candidate regressors (age, sex, smoking, hypertension, hyperlipidemia, diabetes and 
prior stroke). All statistical analyses were performed using the $\mathrm{R}$ software environment for statistical computing and graphics. Statistical significance was taken at the $5 \%$ alpha level.

\section{Results}

\section{Clinical Characteristics}

Forty-five subjects (26 ischemic stroke patients and 19 control subjects) were recruited for this study. All stroke patients were confirmed definite ischemic stroke by neuroimaging (CT or MRI).[16] There was no difference in gender between the groups; however, stroke patients were significantly older $(p<0.001)$. Hypertension $(p=0.007)$ and smoking $(p=0.014)$ were more common in the stroke group compared to controls. The mean NIHSS score of the stroke patients at baseline ( $<24$ hrs. after symptom onset) was 8.65 (range $0-28, S D=7.26)$. Of the 26 strokes, 11 were mild (42\%), 6 were moderate $(23 \%)$, and 9 were scored as severe (35\%). Ten stroke patients (38\%) received rTPA; however, all patients had blood drawn prior to rTPA administration or other intervention. The mean time from symptom onset/"last known normal" was 7 hours and 30 min (Table 1).

\section{ARG1 mRNA Expression, NLR and Stroke Severity}

Stroke severity was analyzed as both NIHSS and infarct volume. ARG1 was positively correlated with NLR ( $r=0.57, p=0.003)$, neutrophil count $(r=0.526, p=0.007)$, NIHSS $(r=0.607, p=0.001)$ and infarct volume $(r=0.27, p=0.051)$ (Figure 1). Although the NLR increases as the NIHSS increases, this was not statistically significant ( $p$-value $=0.263$ ). 
In contrast to the NIHSS, NLR was positively correlated with infarct volume $(r=0.35, p=$ $0.002)$.

Of the 5 genes in our panel, $A R G 1$ was the only gene that was significantly correlated with NIHSS $(r=0.708, p<0.001)$. While the other four genes were not significantly correlated with NIHSS, several of the genes were significantly correlated with ARG1 expression. Both LY96 $(\mathrm{r}=0.3, \mathrm{p}=0.048)$ and MMP9 $(\mathrm{r}=0.646, \mathrm{p}<0.001)$ expression were positively correlated with $A R G 1$ expression. CCR7 expression was negatively correlated with $A R G 1(\mathrm{r}=-0.381, \mathrm{p}=0.011)$.

After AIC model selection there was a statistically significant association between $A R G 1$ and NLR ( $\mathrm{p}=0.019)$, adjusted for age, hypertension, and diabetes. There was also a statistically significant relationship between $A R G 1$ and NIHSS ( $\mathrm{p}=0.001)$, adjusting for age, sex, diabetes, prior stroke, and heart disease. After accounting for the adjusting variables, ARG1 explains $74.19 \%$ of the variation in the reported NIHSS scale. Adjusting for prior stroke, there was also a moderate relationship between the NLR and NIHSS $(p=0.078)$. The median NLR was slightly higher in the moderate/severe stroke patients compared to mild, but not statistically significant (Mean NLR mild $=3.3, \quad N L R$ moderate/severe $=4.5$ ).

Prior to adjusting for regressors, there was not a statistically significant relationship between NLR and infarct volume $(r=0.347, p=0.082)$. Although a regression model including all regressors suggested a statistically significant association between NLR and infarct volume $(p=0.022)$, the AIC selected model with only NLR $(p=0.006)$ and smoking status ( $p=0.003$ ) explained most of the variation in the infarct volume (Adjusted R2 full model $=0.332$ versus Adjusted R2 AIC selected model $=0.458$ ). After accounting for the 
smoking status of the patient, NLR explains a further $29.2 \%$ of the variation in infarct volume. When adjusting for all regressors, there was no statistically significant relationship between $A R G 1$ and infarct volume $(p=0.627)$; this result held in the AIC selected model.

Serum ARG1 Protein Activity, NLR and Stroke Severity

Serum ARG1 protein activity is significantly correlated with whole blood ARG1 mRNA expression ( $\mathrm{r}=0.502, P=0.11)$. Similar to $A R G 1 \mathrm{mRNA}$, there is a significant relationship between serum ARG1 protein activity and stroke severity, as measured by NIHSS. There is a moderately significant unadjusted relationship between serum ARG1 protein activity and NIHSS ( $p=0.15$ ) (Figure 2). After adjusting for age, hypertension, smoking, heart disease, sex, hyperlipidemia, and diabetes, we observe that serum ARG1 activity has a statistically significant association with NIHSS $(p=0.027)$. After AIC model selection there was a statistically significant association between serum ARG1 protein activity and NIHSS $(p=0.005)$, adjusted for heart disease and prior stroke.

There is a statistically significant positive correlation between serum ARG1 protein activity and NLR ( $p=0.01$ ) (Figure 2). After adjusting for confounders, a moderately significant relationship between serum ARG1 activity and NLR remains $(p=0.062)$. After AIC model selection, adjusting for age, hypertension, and diabetes, there is a statistically significant relationship between serum ARG1 activity and NLR $(p=0.009)$.

Gene Profile Validation in Stroke vs. Control 
Relative mRNA expression of $A R G 1, M M P$, and s100a12 was significantly increased in whole blood of stroke patients within 24 hours of symptom onset compared to control (Relative ARG1 expression $=3.4 \pm 3.2, p=0.004$; Relative MMP9 expression $=3.2 \pm 2.8$, $p=0.004$ : Relative s100a12 expression $=2.2 \pm 1.5, p=0.006$ ). Relative mRNA expression of $C C R 7$ was significantly decreased in whole blood of stroke patients within 24 hours of symptom onset compared to control (Relative CCR7 expression $=0.4 \pm 0.32, p=0.03$ ). There was no significant difference in relative mRNA expression of $L Y 96$ between stroke and control (Figure 3). After AIC model selection ARG1 mRNA expression remained significantly higher in stroke compared to control $(p=0.001)$, adjusting for sex, diabetes, and prior stroke.

Principal Component Analysis (PCA) and Principal Component Regression (PCR) PCA with varimax rotation identified three principal components that explain $84.4 \%$ of variation in the original patient gene dataset comprised of $A R G 1, L Y 96, M M P 9$, s100a12, and $C C R 7$ (Table 2). With $A R G 1$ and MMP9 featuring large positive loadings, and a large negative loading for CCR7, the first principal component (PC) can be considered a weighted contrast between ARG1 and MMP9 against CCR7; it explains $38.7 \%$ of total variance (Table 2). The second PC is interpreted as a weighted average of $L Y 96$ and CCR7 and explains $24.4 \%$ of the variation. The third PC is dominated by s100a12 and is interpreted as the contribution of s100a12 explaining $21.3 \%$ of the variation.

Principal component regression (PCR) was performed using the first three components and each outcome of interest: stroke versus control (non-stroke), NIHSS score, NLR, and infarct volume. Each PCR used all three of the derived components as 
regressors. The first PC was statistically significant in the PCR models with stroke versus control $(p=0.002)$, NIHSS $(p<0.001)$ and NLR $(p=0.005)$; it had moderate explanatory utility for infarct volume $(p=0.062)$. The second component was statistically significant in the PCR models with stroke versus control $(p=0.012)$ and infarct volume $(p=0.043)$. The third component was not statistically significant in any of the four PCR models.

\section{Discussion}

The primary purpose of this study was to examine the relationship between ARG1, AIS severity, and immune status as measured by the NLR. We hypothesized that increased ARG1 expression is associated with greater AIS severity and immune dysfunction, as measured by an increased NLR.

This is the first report of a relationship between ARG1 and AIS severity. This study is the first to build upon existing literature which has shown that $A R G 1$ mRNA expression is increased in the peripheral blood, specifically by neutrophils, of AIS patients compared to control subjects; however, the association between ARG1 and increased severity had not yet been examined in the context of AIS. In support of our hypothesis, we observed a statistically significant relationship between increased ARG1, both at the gene and serum protein level, and increased AIS severity, as measured by both NIHSS and infarct volume.

In addition to the relationship between ARG1 expression and AIS severity, we also demonstrate a novel relationship between ARG1 expression and NLR. Here we report that increased ARG1 expression, at both the gene and serum protein level, is positively correlated with NLR and an increased neutrophil count. These associations were 
expected given the previous finding that $A R G 1$ mRNA expression is highest in the neutrophil fraction compared to other leukocyte populations: ${ }^{[5]}$ Surprisingly, we report no relationship between ARG1 expression and lymphocyte count. It has been demonstrated previously that ARG1 protein released from neutrophils suppresses lymphocyte proliferation both in a human ex-vivo model[10], as well as a murine AIS model.[11] Our results indicate that changes in ARG1 expression do not result in significant changes in lymphocyte count. This finding may be explained by the use of a single lymphocyte count, obtained within 24 hours of AIS symptom onset. It may be possible that lymphocyte suppression measured via lymphocyte count may not be detectable until a later time point.[4] As ARG1 inhibits proliferation, there may not be an immediate change in cell count; rather a prolonged suppression of lymphocyte proliferation would result in a reduced count over time.

Given earlier findings by our laboratory, which demonstrate that an elevated NLR is associated with a poor outcome within 90 days of AIS, we hypothesized that the NLR may have a relationship with AIS severity within 24 hours of symptom onset [8]. To address this hypothesis, we examined the relationship between NLR and AIS severity, measured by both NIHSS and infarct volume. While the NLR was higher in severe stroke (NIHSS $>10)$ compared to mild $(\mathrm{NIHSS}<6)$, there was no statistically significant relationship between the NLR and NIHSS. In contrast, there was a statistically significant relationship between the NLR and infarct volume. These discrepant findings between two measures of AIS severity are not contradicting. The NIHSS provides a standardized assessment of neurologic deficit based on changes in cognitive and motor function, and in this study and previous, we have shown that NIHSS and infarct volume are not always 
correlated.[17] This is especially true in AIS patients with posterior circulation strokes, where very small infarcts can result in large neurological deficits and are reflected by higher NIHSS scores. The strong relationship between the NLR and infarct volume indicates that the magnitude of the immune response, as well as the factors that control that response, are a reflection of the amount of brain tissue compromised, rather than stroke-related symptom manifestation. In this study, the infarct volume was calculated based on imaging obtained near the time of blood sampling. Future studies will address how changes in both the NLR, as well as the factors that mediate the NLR, including ARG1, contribute to the evolution of brain infarct over time after AIS.

We validated the use of a gene profile comprised of five genes, ARG1, LY96, MMP9, s100a12, and CCR7 to diagnose AIS. This validation was necessary given that previous validation studies utilized the reference gene $\beta$-actin, however, $\beta$-actin has been recently shown to be an unreliable reference gene, due to variable expression levels in AIS.[13] We utilized a combination of two genes: B2M and PPIB that have been confirmed to be stably expressed in AIS. Compared to our previous study that had identified this panel of five genes associated with AIS, the relative expression of only one gene, $L Y 96$, was not increased in AIS patients in this separate cohort. The relative expression of the four remaining genes $A R G 1, M M P 9$, s100a12, and CCR7 was comparable to previous studies.

In addition to validating the use of this gene profile in the diagnosis of AIS, we utilized PCA to examine the relationship between this panel of genes, AIS severity, and, the NLR. We hypothesized that the pattern of expression amongst these genes would have better predictive value than studying single biomarkers alone. PCA represents an 
underutilized technique for studying the complex interactions giving rise to post-stroke immunosuppression. PC1 featured large positive loadings for ARG1 and MMP9 and a large negative loading score for $C C R 7$, indicating an inverse relationship between $A R G 1$ and MMP9 expression and $C C R 7$ expression. Further, $\mathrm{PC} 1$ is significantly correlated with AIS severity and NLR, and the relationships between PC1, AIS severity, and NLR, are stronger than the relationships between ARG1, AIS severity, and NLR. These findings imply that recognizing patterns of biomarkers provides more clinical information than single biomarkers alone. While PCA is merely statistical, a potential physiological mechanism may be proposed based on these results. We postulate that the combination of increased $A R G 1$ expression and decreased $C C R 7$ expression results in an increased NLR. Because CCR7 expression mediates lymphocyte migration to secondary lymphoid organs and lymphocyte activation, reduced CCR7 expression may represent a mechanism of $\mathrm{T}$ lymphocyte suppression, mechanistically similar to the downregulation of $\mathrm{CD} 3 \zeta$ chain.[18] Future studies, including human ex-vivo and animal models of AIS, will be necessary to examine the functional relationship between $A R G 1$ and $C C R 7$.

There are several limitations of this study that need to be addressed. The first limitation is the relatively modest sample size compared to other genomic biomarker studies. While the sample population in this study is limited, this sample size provides the required statistical power to address the primary aims of this study. The second limitation is the statistically significant age difference between the AIS and control groups, and the large age range within the AIS group. To consider the understanding that immune system function diverges with advancing age[19], we used linear regression analysis to statistically control for age, and demonstrated that the relationships between ARG1, AIS 
severity, and NLR remain significant when accounting for age. This suggests that while the contribution of age to these relationships exists, there are other variables that are more significant contributors to these relationships. In addition to the large age range in the stroke subgroup, we did not acquire TOAST classification to determine AIS etiology.

This is the first report of a relationship between ARG1, infarct volume, and NLR in a human AIS model. This is also the first study to utilize PCA to model post-stroke immunosuppression. PCA is an underutilized technique that has clinical utility in the study of complex diseases, including AIS, and using PCA analysis we discovered novel relationships between multiple biomarkers that may play a physiological role in poststroke immune suppression. The findings presented in this study have several implications for future clinical practice. ARG1 inhibition recently entered clinical trials for use in ischemia-reperfusion injury in patients with coronary artery disease.[20] Given the similar pathophysiological consequences in myocardial ischemia-reperfusion injury and AIS, it is plausible that ARG1 inhibition may represent a promising potential immune modulating treatment following AIS. Furthermore, because post-stroke immunosuppression persists for a period time in AIS recovery, ARG1 inhibition may have a beneficial effect on AIS severity and outcome even administered at a later time point than recanalization agents, such as rTPA.

\section{Conclusion}

Our study suggests a novel relationship exists between ARG1, NLR and stroke severity which may help guide future mechanistic studies of post-stroke immune suppression. The NLR is an under-utilized clinically available biomarker to monitor the post-stroke immune 
response. Future studies will need to address the functional relationships between ARG1 and NLR post-stroke to test novel immune modulating therapeutic strategies for stroke patients. 


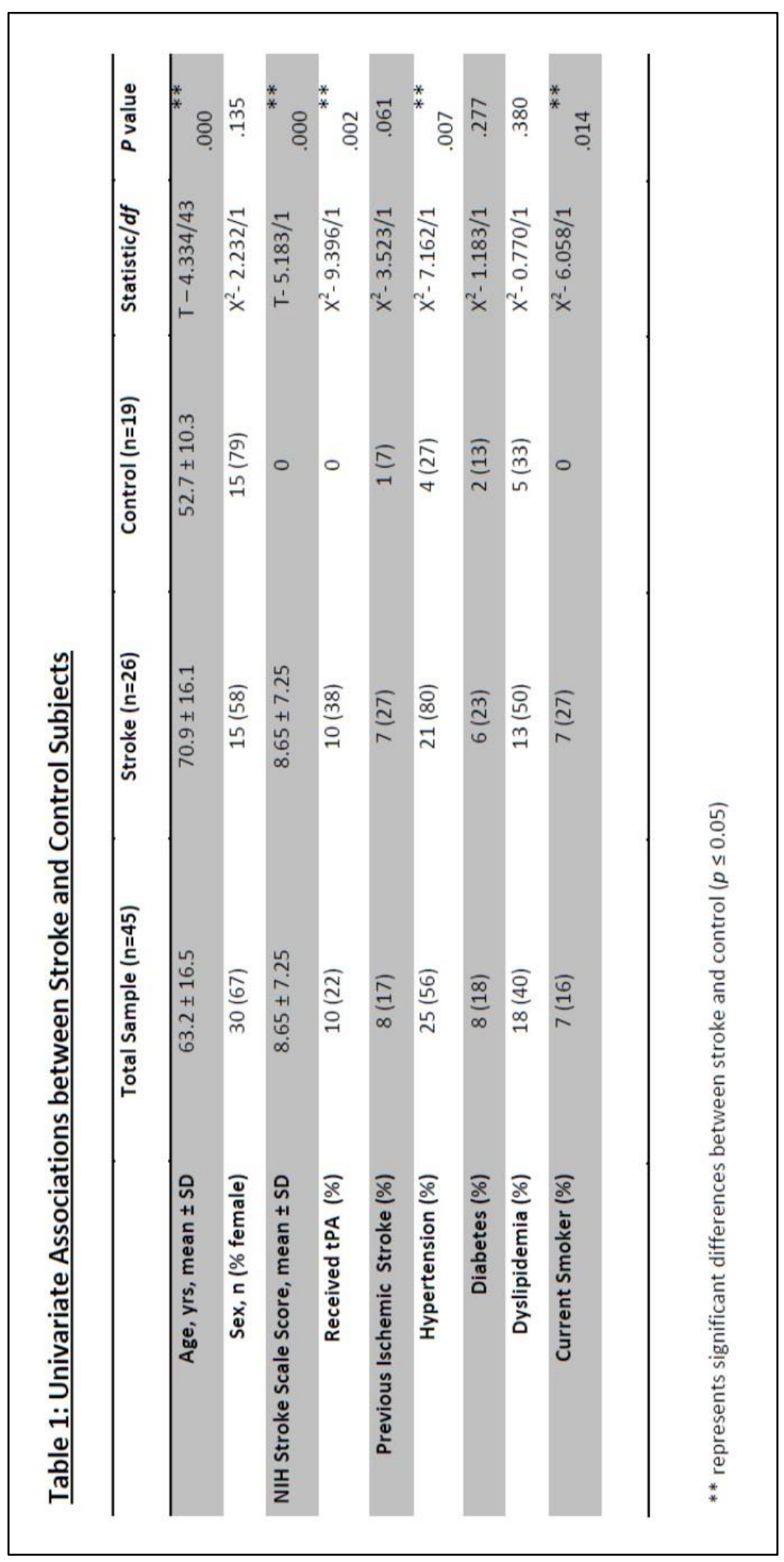


Table 2: Loadings from the principal component analysis with varimax rotation using the five genes of interest: ARG1, LY96, MMP9, s100a12, and CCR7.

\begin{tabular}{|l|l|l|l|}
\hline & PC 1 & PC 2 & PC 3 \\
\hline arg1 & 0.890 & 0.229 & -0.153 \\
\hline ly96 & 0.307 & 0.875 & -0.003 \\
\hline mmp9 & 0.852 & 0.016 & 0.102 \\
\hline s100a12 & 0.001 & -0.064 & 0.991 \\
\hline ccr7 & -0.570 & 0.630 & -0.218 \\
\hline Explained Variance (\%) & 38.7 & 24.4 & 21.3 \\
\hline Cumulative Variance Explained (\%) & 38.7 & 63.1 & 84.4 \\
& & & \\
\hline
\end{tabular}



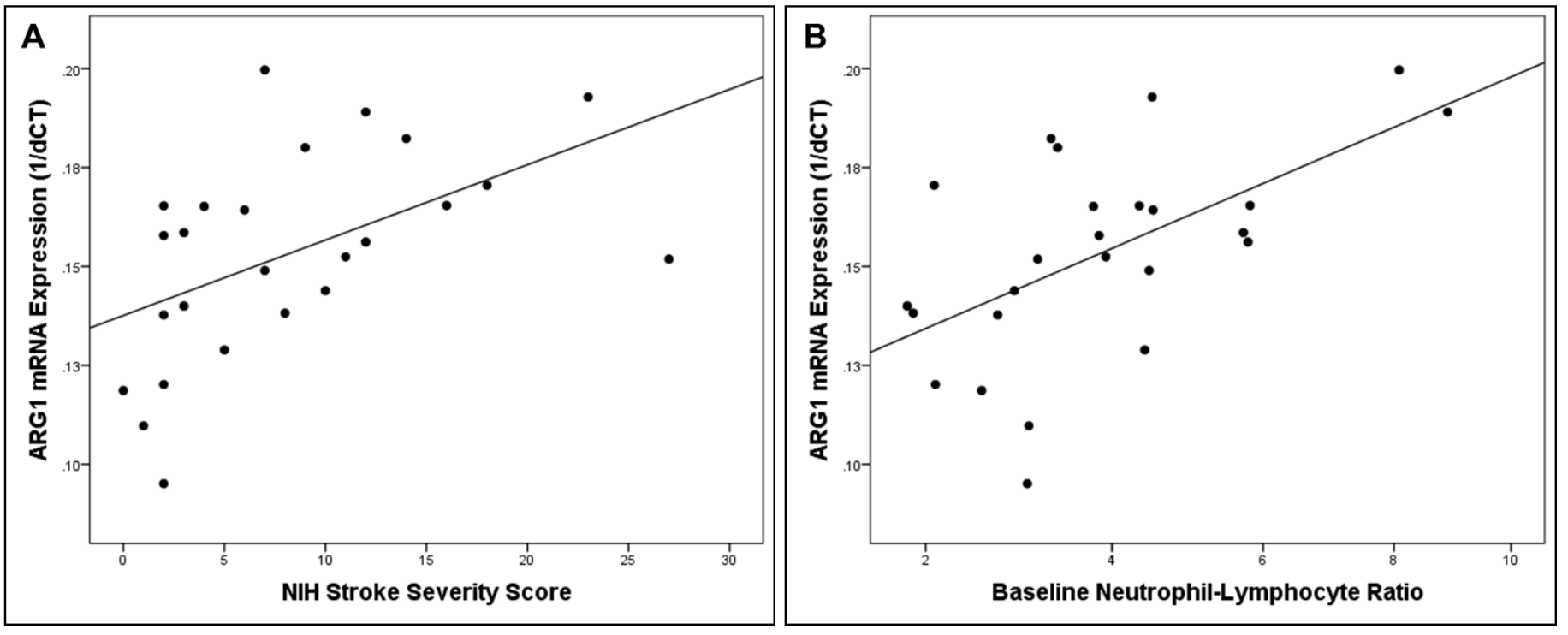

Figure 1: The Relationship between ARG1 mRNA Expression, NIHSS, and NLR.

(A) ARG1 Expression is positively correlated with NIHSS ( $r=0.607, p<0.001)$.

(B) ARG1 Expression is positively correlated with NLR ( $r=0.582, p=0.002)$. 

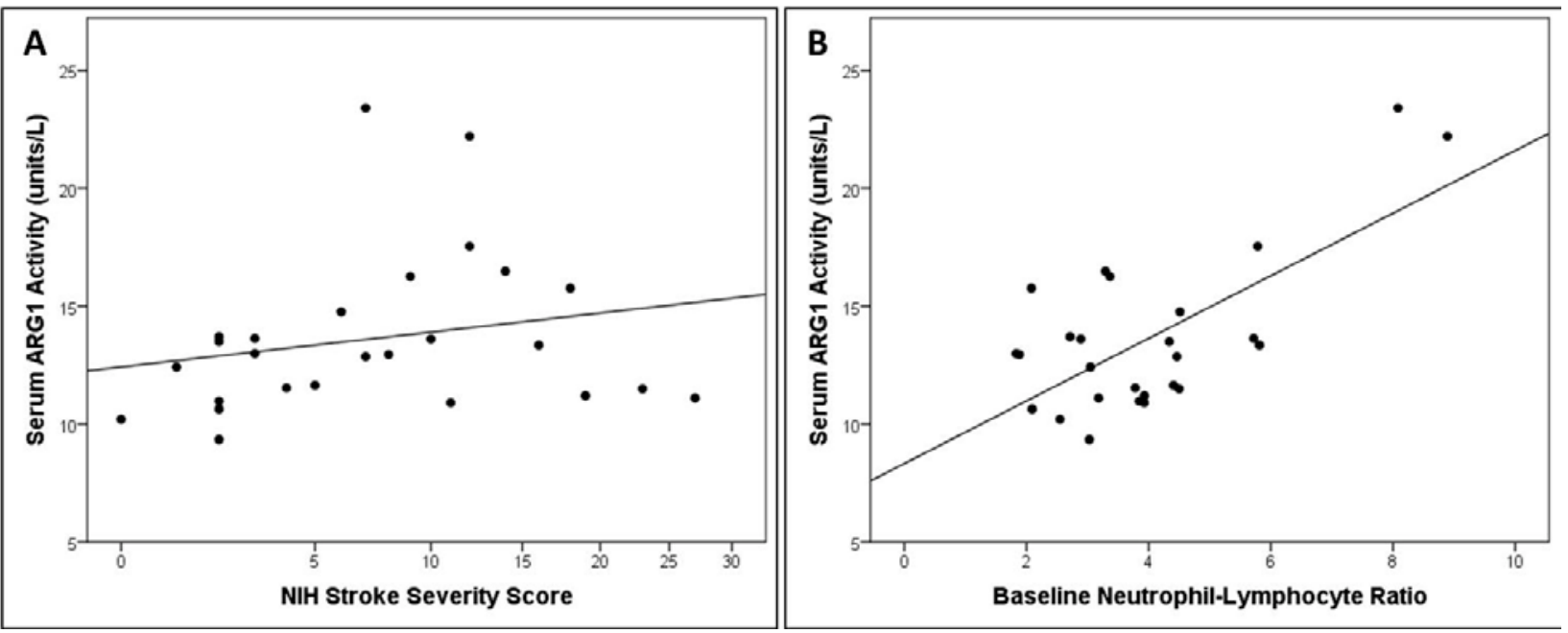

Figure 2: The Relationship between Serum ARG1 Protein Activity, NIHSS, and NLR.

(A) Serum ARG1 Protein Activity is positively correlated with NIHSS ( $r=0.31, p=0.15)$.

(B) Serum ARG1 Protein Activity is positively correlated with NLR ( $r=0.52, p=0.01)$. 


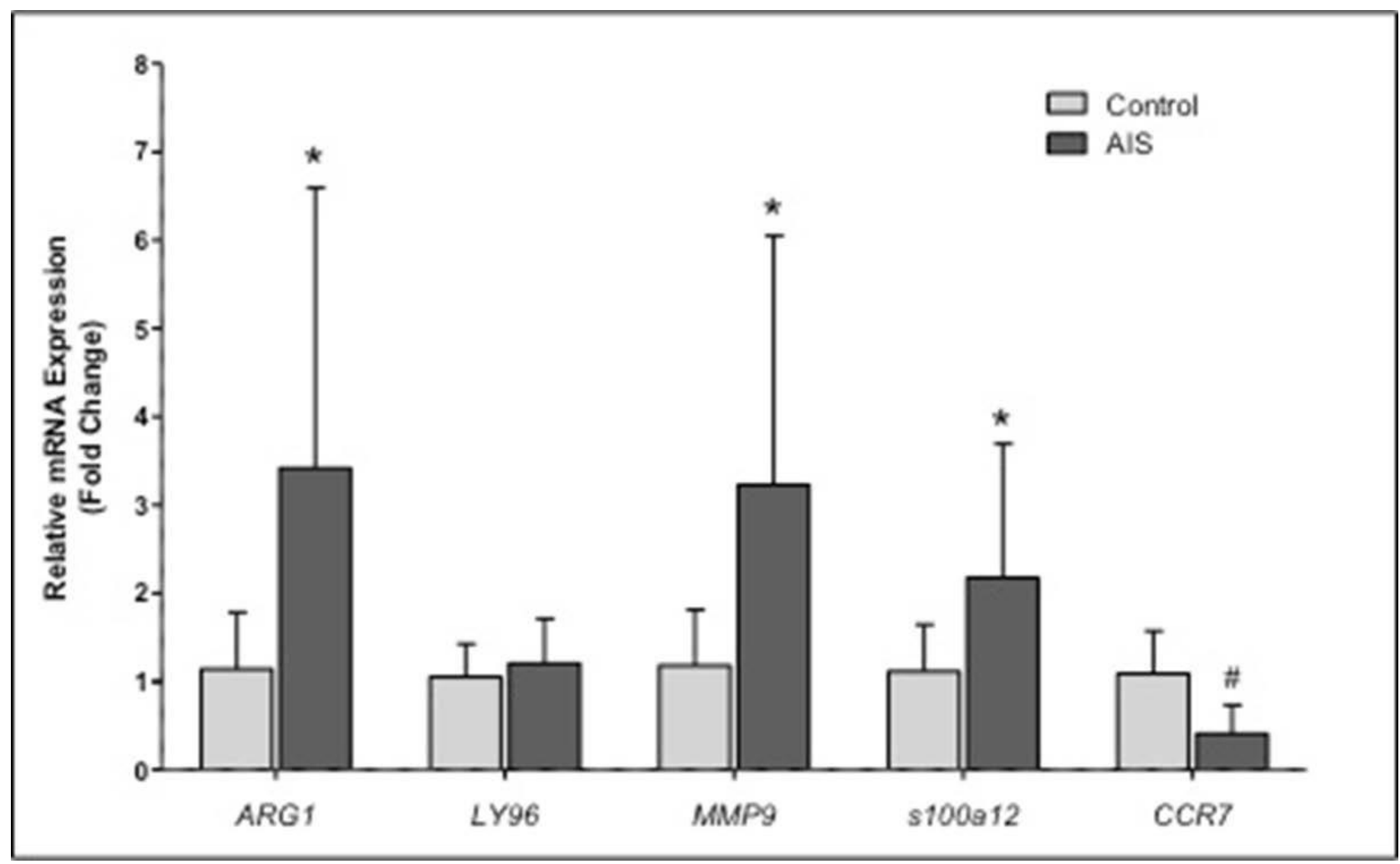

Figure 3: Relative Expression of ARG1, LY96, MMP9, s100a12, and CCR7 in AIS. ARG1, MMP9, and s100a12 expression are significantly increased in whole blood of AIS patients compared to control. CCR7 expression is significantly decreased in whole blood of AIS patients compared to control. * denotes significant increases in gene expression in AIS ( $p<0.05)$, \# denotes significant decreases in gene expression in AIS $(p<0.05)$.

\section{References}


1. Vogelgesang A, Dressel A. Immunological consequences of ischemic stroke: immunosuppression and autoimmunity. Journal of neuroimmunology. 2011;231(1-2):10510.

2. Kamel H, ladecola C. Brain-immune interactions and ischemic stroke: clinical implications. Archives of neurology. 2012;69(5):576-81.

3. Shichita T, Sakaguchi R, Suzuki M, Yoshimura A. Post-ischemic inflammation in the brain. Frontiers in immunology. 2012;3:132.

4. Offner $\mathrm{H}$, Vandenbark AA, Hurn PD. Effect of experimental stroke on peripheral immunity: CNS ischemia induces profound immunosuppression. Neuroscience. 2009;158(3):1098-111.

5. Tang Y, Xu H, Du X, Lit L, Walker W, Lu A, et al. Gene expression in blood changes rapidly in neutrophils and monocytes after ischemic stroke in humans: a microarray study. Journal of cerebral blood flow and metabolism : official journal of the International Society of Cerebral Blood Flow and Metabolism. 2006;26(8):1089-102.

6. Barr TL, Conley Y, Ding J, Dillman A, Warach S, Singleton A, et al. Genomic biomarkers and cellular pathways of ischemic stroke by RNA gene expression profiling. Neurology. 2010;75(11):1009-14.

7. Tokgoz S, Kayrak M, Akpinar Z, Seyithanoglu A, Guney F, Yuruten B. Neutrophil lymphocyte ratio as a predictor of stroke. Journal of stroke and cerebrovascular diseases : the official journal of National Stroke Association. 2013;22(7):1169-74.

8. Brooks SD, Spears C, Cummings C, VanGilder RL, Stinehart KR, Gutmann L, et al. Admission neutrophil-lymphocyte ratio predicts 90 day outcome after endovascular stroke therapy. Journal of neurointerventional surgery. 2014;6(8):578-83. 
9. Caldwell RB, Toque HA, Narayanan SP, Caldwell RW. Arginase: an old enzyme with new tricks. Trends in pharmacological sciences. 2015;36(6):395-405.

10. Munder M. Arginase: an emerging key player in the mammalian immune system. Br J Pharmacol. 2009;158(3):638-51.

11. Sippel TR, Shimizu T, Strnad F, Traystman RJ, Herson PS, Waziri A. Arginase I release from activated neutrophils induces peripheral immunosuppression in a murine model of stroke. Journal of cerebral blood flow and metabolism : official journal of the International Society of Cerebral Blood Flow and Metabolism. 2015.

12. Mestas J, Hughes CC. Of mice and not men: differences between mouse and human immunology. Journal of immunology. 2004;172(5):2731-8.

13. Stamova BS, Apperson $\mathrm{M}$, Walker $\mathrm{WL}$, Tian $\mathrm{Y}, \mathrm{Xu} \mathrm{H}$, Adamczy $\mathrm{P}$, et al. Identification and validation of suitable endogenous reference genes for gene expression studies in human peripheral blood. BMC medical genomics. 2009;2:49.

14. Livak KJ, Schmittgen TD. Analysis of relative gene expression data using real-time quantitative PCR and the 2(-Delta Delta C(T)) Method. Methods. 2001;25(4):402-8.

15. Faul F, Erdfelder E, Buchner A, Lang AG. Statistical power analyses using G*Power 3.1: tests for correlation and regression analyses. Behavior research methods. 2009;41(4):1149-60.

16. Kidwell CS, Warach S. Acute ischemic cerebrovascular syndrome: diagnostic criteria. Stroke; a journal of cerebral circulation. 2003;34(12):2995-8.

17. Woo D, Broderick JP, Kothari RU, Lu M, Brott T, Lyden PD, et al. Does the National Institutes of Health Stroke Scale favor left hemisphere strokes? NINDS t-PA Stroke Study Group. Stroke; a journal of cerebral circulation. 1999;30(11):2355-9. 
18. Moschovakis GL, Forster R. Multifaceted activities of CCR7 regulate T-cell homeostasis in health and disease. European journal of immunology. 2012;42(8):194955.

19. Ponnappan S, Ponnappan U. Aging and immune function: molecular mechanisms to interventions. Antioxidants \& redox signaling. 2011;14(8):1551-85.

20. Tratsiakovich Y, Yang J, Gonon AT, Sjoquist PO, Pernow J. Arginase as a target for treatment of myocardial ischemia-reperfusion injury. European journal of pharmacology. 2013;720(1-3):121-3. 


\title{
Chapter 3
}

The Relationship Between Acute Changes in Neutrophil-Lymphocyte Ratio in Ischemic Stroke Patients on Stroke Severity and Outcome

\author{
Ashley B. Petrone, BS ${ }^{1,2}$ \\ ${ }^{1}$ Dept. of Neurobiology and Anatomy, and ${ }^{2}$ Center for Basic and \\ Translational Stroke Research \\ West Virginia University, One Medical Center Drive, \\ Morgantown WV 26506
}


Background: Acute ischemic stroke (AIS) induces activation of both the innate and adaptive arms of the immune system. While this immune response is critical to resolution of ischemic damage in the brain, these alterations in immune function can affect systemic immune function outside of the brain. This altered peripheral immune response has been termed post-stroke immunosuppression and contributes to increased stroke severity, increased rate of infection, and overall poorer recovery following stroke. Our laboratory has shown that the baseline neutrophil-lymphocyte ratio (NLR) is a simple, inexpensive indicator of post-stroke immunosuppression that may be used to predict outcome following AIS. The aim of this study was to expand upon these findings and determine if measuring the NLR after thrombolysis or at several time points, rather than at baseline alone, may provide greater insight into stroke severity and outcome. We hypothesize that an increase in NLR following thrombolysis is associated with increased severity and poor outcome, regardless of the baseline NLR value prior to treatment.

Methods: AIS patients $(n=18)$ of varying severity, as measured by NIHSS, were recruited for this study. NLR was calculated at baseline shortly after admission, prior to intervention. NLR was measured at a second time point following thrombolysis, approximately 24 hours following the baseline NLR. The changes in NLR ( $\triangle N L R)$ over 24 hours were compared between mild, moderate, and severe AIS groups.

Results: There were no significant differences in $\triangle$ NLR over 24 hours between AIS severity groups; however, there were observable trends in the data. The mean $\triangle N L R$ in the mild AIS group was -0.05 , compared to +2.2 and +2.8 in the moderate and severe AIS groups, respectively.

Conclusions: This preliminary analysis provides support for the hypothesis that measuring the NLR at a time point following thrombolysis or $\triangle \mathrm{NLR}$ from baseline may provide more insight into AIS patient recovery. This analysis also reaffirmed our previous finding that baseline NLR may be used to stratify AIS patients by severity upon admission.

\section{Introduction}


Acute ischemic stroke (AIS) induces activation of both the innate and adaptive arms of the immune system $[1,2]$. Neutrophils are the first cells to migrate from the secondary lymphoid organs and circulation to the brain following AIS $[1,2]$. Following a period of inflammation as a result of neutrophil infiltration, the cells of the adaptive immune response, specifically $\mathrm{T}$ lymphocytes, are activated and migrate into the brain to elicit an antigen-specific immune response to the ischemic brain region $[2,3]$. While this immune response is critical to resolution of ischemic damage in the brain, these alterations in immune function can affect systemic immune function outside of the brain. This altered peripheral immune response has been termed post-stroke immunosuppression, and while the exact mechanisms that contribute to post-stroke immunosuppression are unclear, this period of altered immune function is characterized by increased antiinflammatory cytokines, lymphopenia, and splenic atrophy $[2,3]$. Clinically, post-stroke immunosuppression contributes to increased stroke severity, increased rate of infection, and overall poorer recovery following stroke $[2,3]$. Our laboratory has shown that the neutrophil-lymphocyte ratio (NLR) is a simple, inexpensive indicator of post-stroke immunosuppression that may be used to predict outcome following AIS [4-7]. To date, all of the studies evaluating the role of the NLR as a prognostic factor in AIS have examined the NLR at a single time point within 24 hours of stroke symptom onset. While baseline NLR is correlated with baseline stroke severity and also associated with poor outcome and increased mortality within 90 days of AIS, we hypothesize that measuring the NLR at an acute time point following thrombolysis, may provide more insight into stroke severity and future outcome than baseline NLR. Specifically, we hypothesize that an increase in 
NLR following thrombolysis is associated with increased severity and poor outcome, regardless of the baseline NLR value prior to treatment.

\section{Methods}

Subject Recruitment

Informed consent was obtained from all individual participants included in this study. AIS patients and stroke-free control subjects were recruited from Ruby Memorial Hospital (Morgantown, WV). Male and female AIS patients were eligible for recruitment if the following inclusion criteria were met: (1) age $\geq 18$ years, (2) confirmation of acute stroke by neuroimaging (CT or MRI), and (3) had blood drawn within 24 hours of symptom onset/"last-known normal", prior to thrombolysis or interventional treatment. Patient data from the medical record were reviewed and recorded, including (1) National Institutes of Health Stroke Scale (NIHSS), (2) clinical laboratory analyses, including white blood cell differential, and (3) brain imaging (CT/ MRI). Outcome was assessed by Modified Rankin Scale (MRS) obtained approximately 30 days post-AIS. MRS $<2$ were considered as favorable outcomes, whereas MRS $\geq 2$ were considered poor outcomes.

\section{Neutrophil-Lymphocyte Ratio}

Absolute neutrophil and lymphocyte counts were derived from white blood cell differentials, and the NLR was calculated as the absolute neutrophil count divided by absolute lymphocyte count. White blood cell differentials were performed upon admission, prior to thrombolysis or intervention, and repeated approximately 24 hours later. $\triangle N L R$ was calculating by subtracting the NLR baseline from NLR $24 \mathrm{hr}$. 


\section{Statistical Analysis}

A one-way analysis of variance (one-way ANOVA) tests was used to compare the mean NLR and $\triangle \mathrm{NLR}$ between the mild, moderate, and severe AIS severity groups. MannWhitney U Tests were used to compare $\triangle$ NLR between AIS patient groups, straitfied by MRS. The relationship between baseline NLR and baseline NIHSS was tested using Spearman's rank-order correlation.

\section{Results}

\section{Clinical Characteristics}

A total of 18 AIS patients were included in this study ( 8 mild, 5 moderate, and 5 severe). There were no significant differences between groups for demographic and comorbities, including age, sex, prior stroke, hypertension, diabetes, and dyslipidemia (Table 1). The mean NIHSS score was significantly different between AIS severity groups (mean mild $\mathrm{NIHSS}=2.3 \pm 0.89 ;$ mean moderate NIHSS $=8.3 \pm 1.26$; mean severe NIHSS $=22 \pm$ 6.39). Ten patients (55\%) received tPA (6 mild, 2 moderate, 2 severe); however, baseline NIHSS and NLR were determined prior to tPA administration.

\section{Baseline NLR and Stroke Severity}

There is a weak unadjusted relationship between baseline NLR and baseline NIHSS $(r=0.336, p=0.17)$ (Figure 1). The mean baseline NLR differs between mild, moderate, and severe AIS severity groups; however, the observed differences are not statistically significant (mild NLR=2.9, moderate NLR $=1.7$, severe NLR=3.8) (Figure 2). 
Change in NLR Over 24 hours ( $\triangle N L R)$ and Stroke Severity

There were no significant differences in $\triangle \mathrm{NLR}$ over 24 hours between AIS severity groups; however, there were observable trends in the data (Figures 3, 4). The mean $\Delta \mathrm{NLR}$ in the mild AIS group was -0.05 , compared to +2.2 and +2.8 in the moderate and severe AIS groups, respectively. There was no difference in $\triangle \mathrm{NLR}$ in patients who received tPA compared to those who did not receive tPA (mean $\triangle N L R$ received tPA $=$ 0.22, $\triangle \mathrm{NLR}$ no tPA $=+1.1$.

Relationship Between baseline NLR, $\triangle N L R$, and Outcome

There were no statistically significant differences in baseline NLR between AIS patients with a favorable outcome (MRS <2) and poor outcome (MRS $\geq 2$ ). Further, there were no statistically significant differences in $\triangle N L R$ between AIS patients with a favorable outcome (MRS <2) and poor outcome (MRS $\geq 2$ ); however, there were greater increases in $\triangle \mathrm{NLR}$ in the poor outcome group compared to several decreases in $\triangle \mathrm{NLR}$ observed in the favorable outcome group (Figure 5).

\section{Discussion}

This preliminary analysis is the first to describe the change in NLR following thrombolysis in AIS patients. The aim of this study was to determine if $\triangle N L R$ was more correlated with stroke severity, as measured by NIHSS, and AIS outcome, as measured by MRS, than baseline NLR alone. While the findings of this study lacked statistical significance, there is evocative evidence to suggest that not only can the baseline NLR be used to stratify AIS patients according to severity, but that measuring the change in NLR following thrombolysis may also be indicative of acute severity, and may be a stronger indicator of 
outcome compared to baseline NLR. While this preliminary analysis may support the use of $\triangle N L R$ as a marker of AIS outcome, there are several limitations of this study that need to be addressed. The most concerning limitation of this study is the extremely small sample size $(n=18)$. Sample size becomes even more concerning when divinding study participants into groups based on NIHSS. Having a small sample size requires practicing caution when interpreting the the results of this study; however, the results of this study support the investigation of this hypothesis in a larger cohort of AIS patients. Future studies should also assess the NLR at later time points during recovery, such as 7 days, 30 days, etc. to provide even more insight into immunosuppression during recovery, as the NLR measurements obtained during this study were taken very shortly following AIS. In summary, while the results of this study should be interpreted cautiously, there is preliminary evidence to suggest that the NLR following thrombolysis in AIS patients may be a more relevant marker of AIS outcome than NLR prior to thrombolysis, and the research questions addressed in this study warrant invesitgation in a larger patient cohort. 


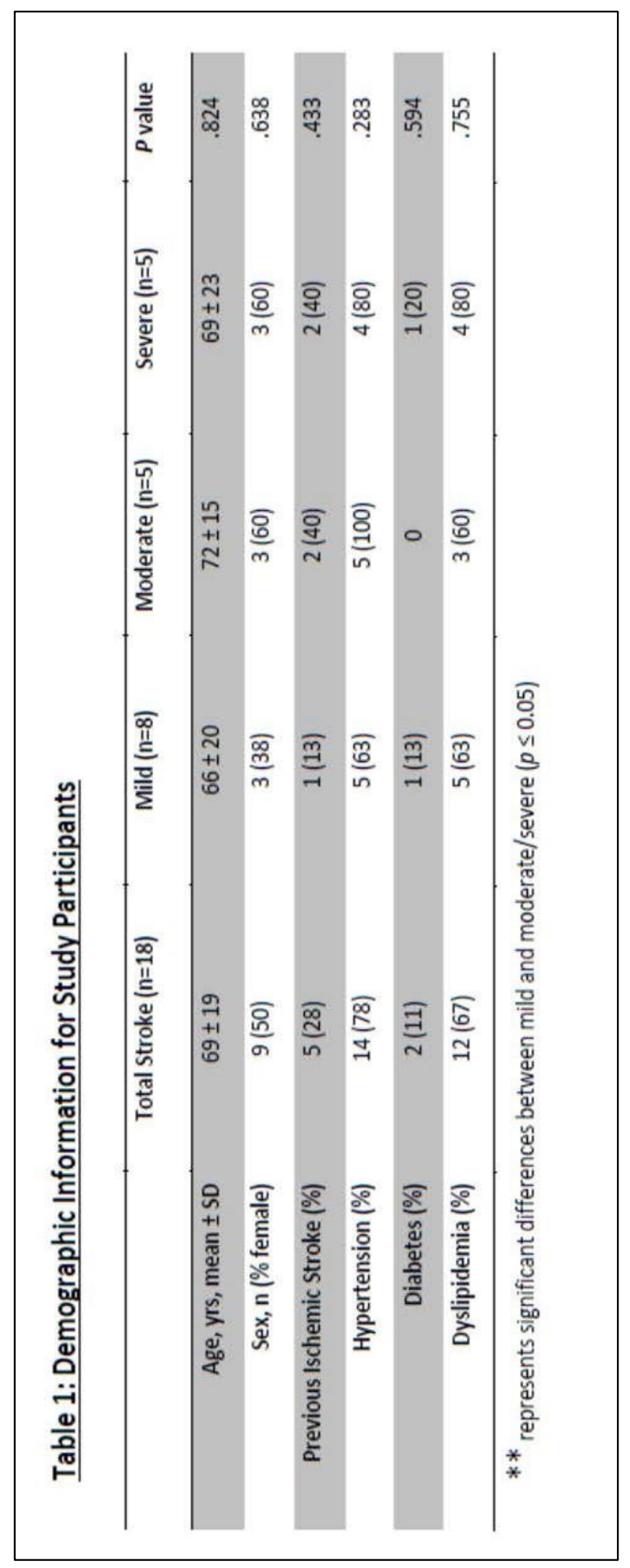




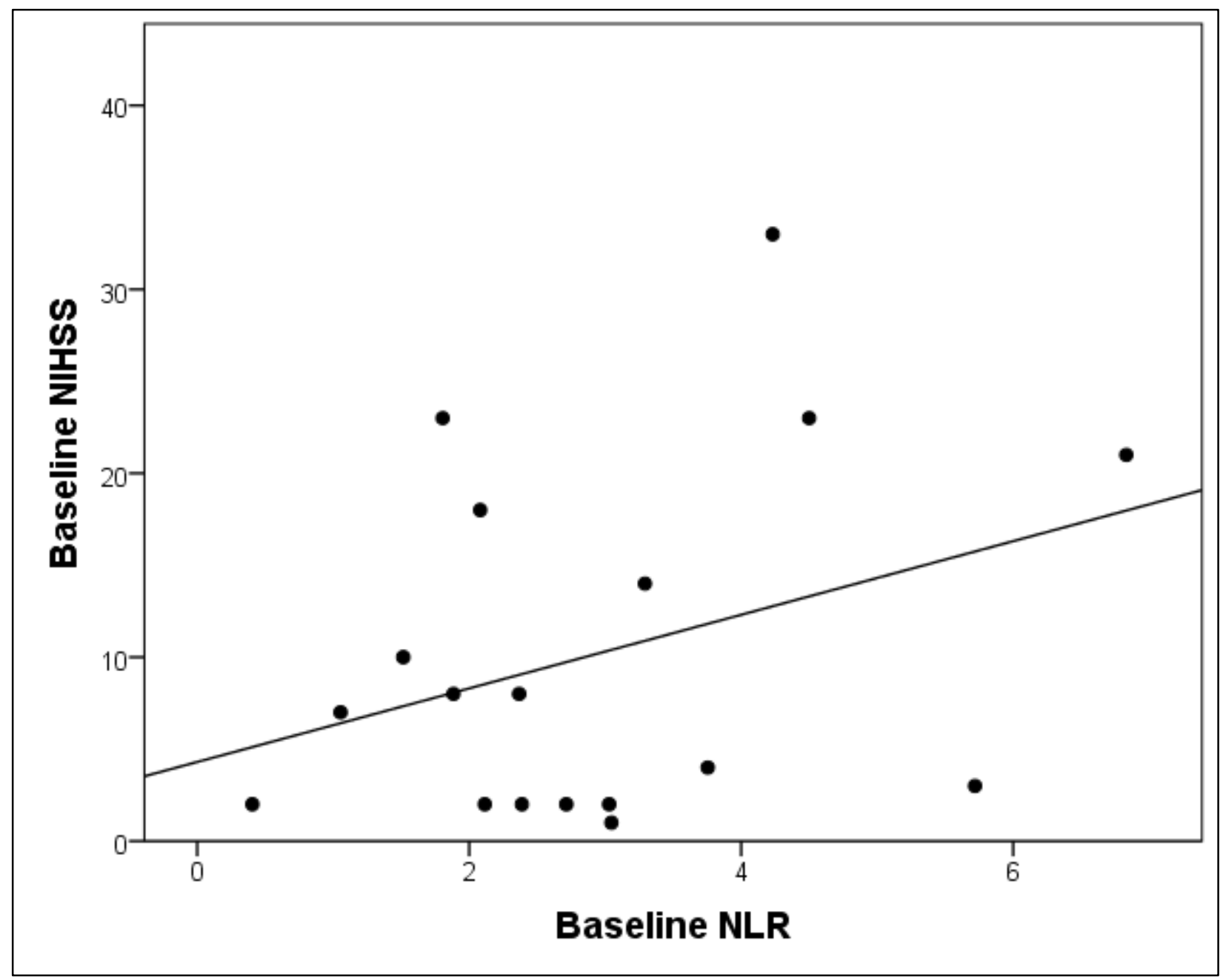

Figure 1: The Relationship Between Baseline NIHSS and Baseline NLR. There is a weak unadjusted relationship between baseline NLR and baseline NIHSS $(r=0.336$, $p=0.17)$. 


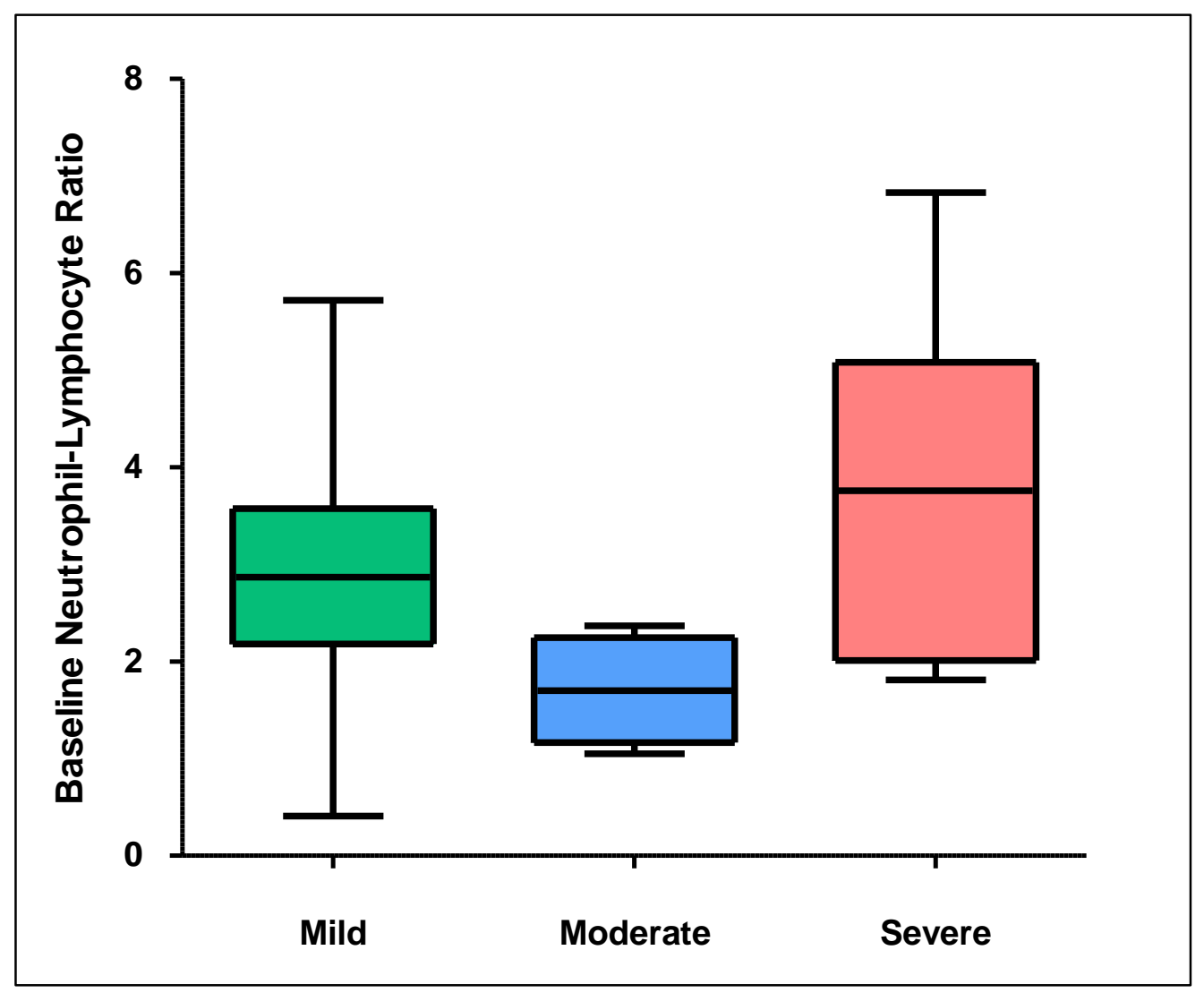

Figure 2: Differences in Baseline NLR among AIS Severity Subgroups. The mean baseline NLR differs between mild, moderate, and severe AIS severity groups; however, the observed differences are not significant (mild NLR $=2.9$ (green), moderate NLR $=1.7$ (blue), severe NLR =3.8 (red)). 


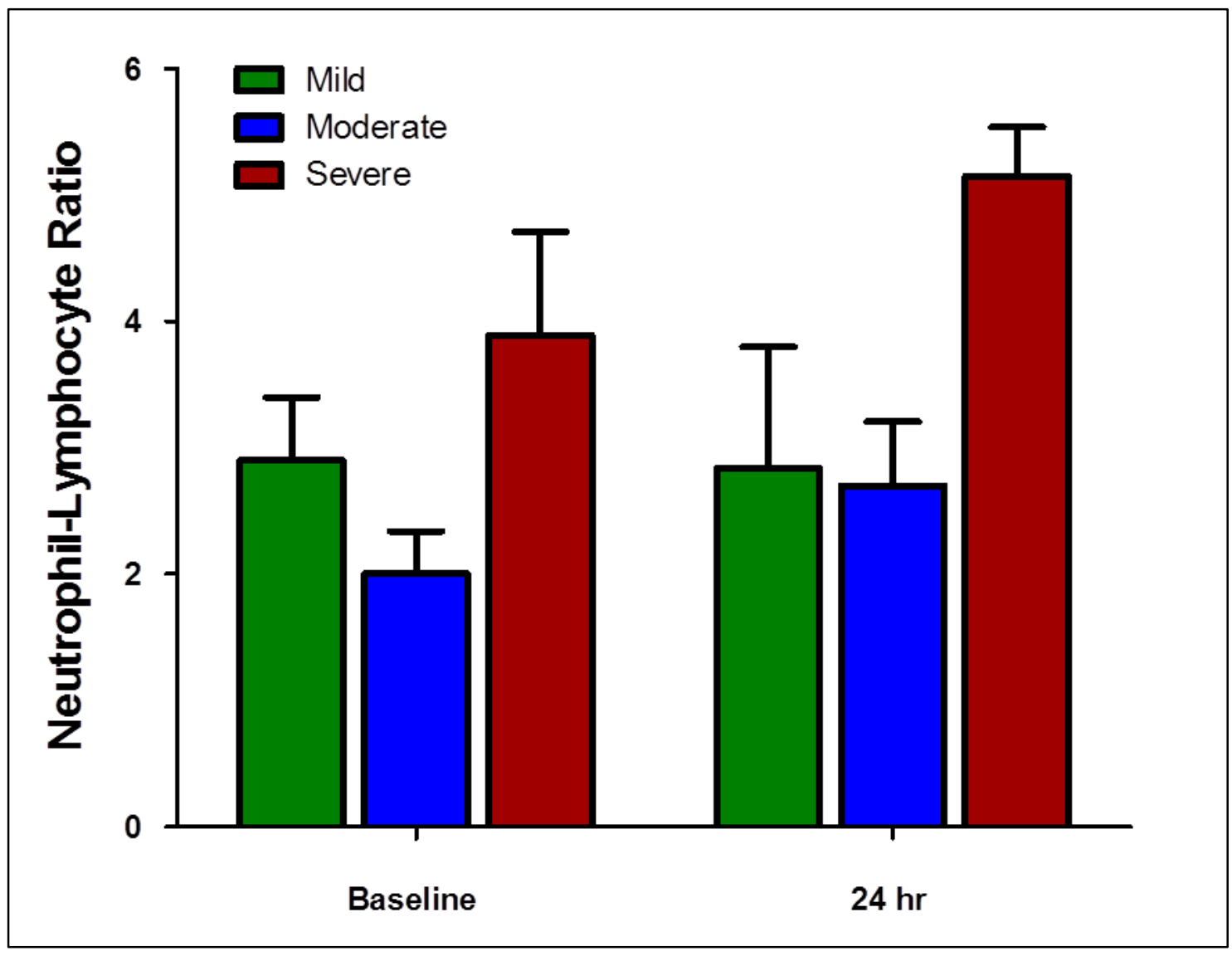

Figure 3: Differences in Baseline and 24 Hour NLR among AIS Severity Subgroups. The mean $\triangle \mathrm{NLR}$ in the mild AIS group (green) was -0.05 , compared to +2.2 and +2.8 in the moderate (blue) and severe (red) AIS groups, respectively. 


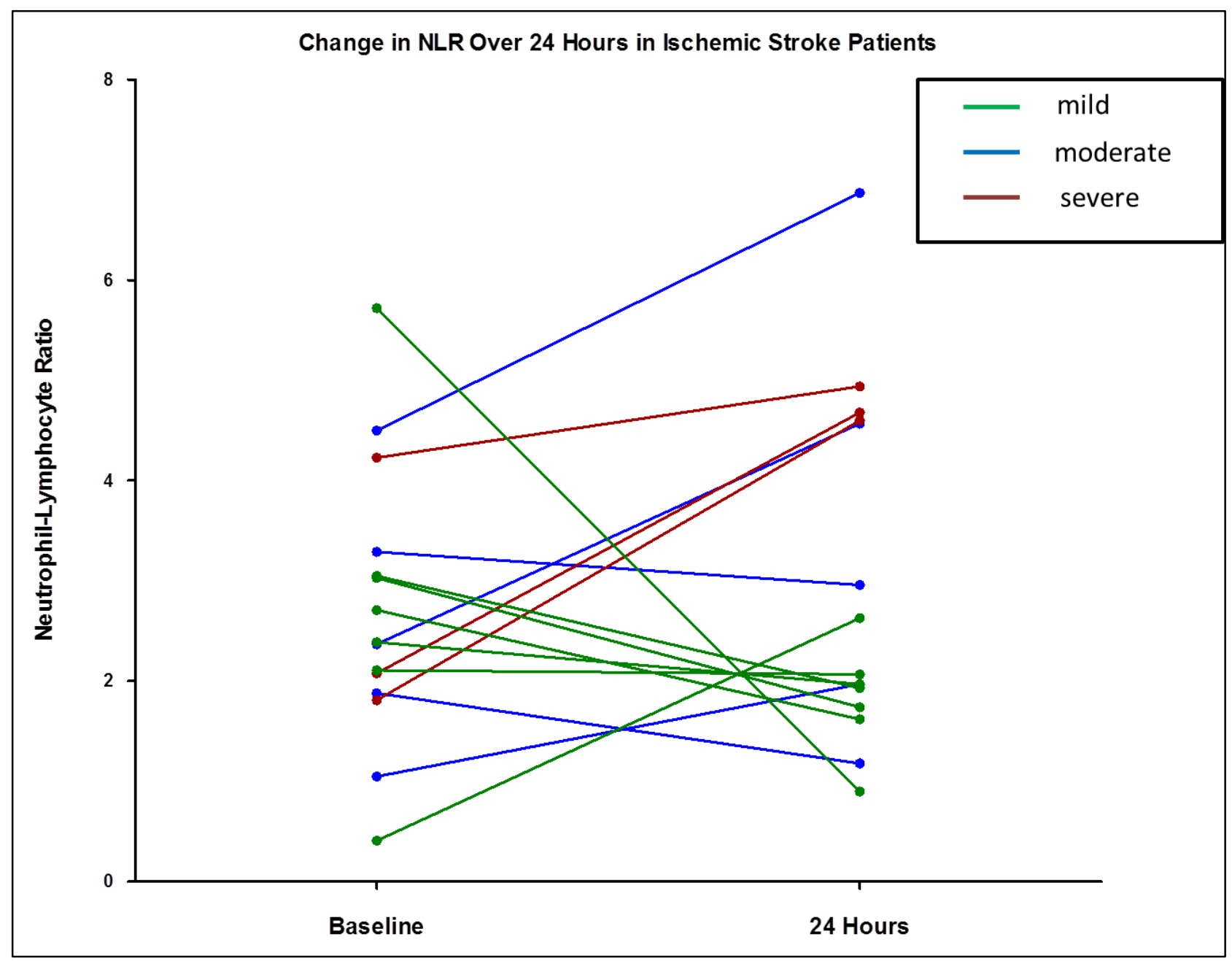

Figure 4: Change in NLR Over 24 hours $(\triangle N L R)$ in Individual Patients in AIS Severity Subgroups. The mean $\Delta \mathrm{NLR}$ in the mild AIS group was -0.05 , compared to +2.2 and +2.8 in the moderate and severe AIS groups, respectively. Mild AIS patients appear to have a decrease in NLR over 24 hours, whereas severe AIS patients show an increased in NLR over 24 hours. 


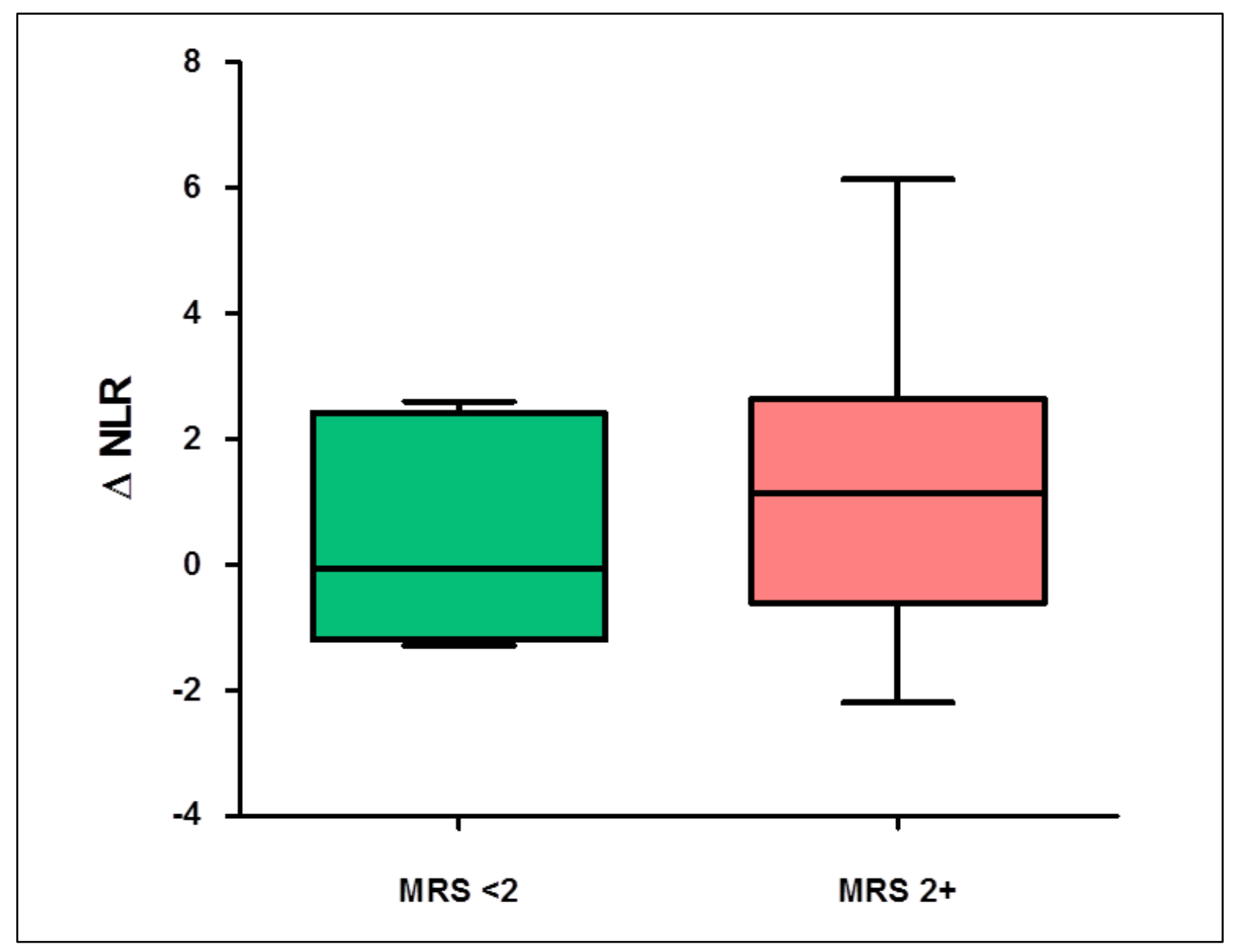

Figure 5: Relationship Between $\triangle \mathrm{NLR}$ and AIS Outcome. There were greater increases in $\triangle N L R$ in the poor outcome group compared to the favorable outcome group (Mean $\triangle \mathrm{NLR}$ favorable outcome $=+0.47$; mean $\Delta \mathrm{NLR}$ poor outcome $=+1.27$ ). 


\section{References}

1. Vogelgesang A, Dressel A. Immunological consequences of ischemic stroke: Immunosuppression and autoimmunity. J Neuroimmunol. 2011;231:105-110

2. Kamel H, ladecola C. Brain-immune interactions and ischemic stroke: Clinical implications. Arch Neurol. 2012;69:576-581

3. Offner $H$, Vandenbark AA, Hurn PD. Effect of experimental stroke on peripheral immunity: $\mathrm{Cns}$ ischemia induces profound immunosuppression. Neuroscience. 2009;158:1098-1111

4. Petrone AB, O'Connell GC, Regier MD, Chantler PD, Simpkins JW, Barr TL. The role of arginase 1 in post-stroke immunosuppression and ischemic stroke severity. Trans/ Stroke Res. 2015

5. Brooks SD, Spears C, Cummings C, VanGilder RL, Stinehart KR, Gutmann L, et al. Admission neutrophil-lymphocyte ratio predicts 90 day outcome after endovascular stroke therapy. J Neurointerv Surg. 2014;6:578-583

6. Tokgoz S, Kayrak M, Akpinar Z, Seyithanoglu A, Guney F, Yuruten B. Neutrophil lymphocyte ratio as a predictor of stroke. J Stroke Cerebrovasc Dis. 2013;22:1169-1174

7. Tokgoz S, Keskin S, Kayrak M, Seyithanoglu A, Ogmegul A. Is neutrophil/lymphocyte ratio predict to short-term mortality in acute cerebral infarct independently from infarct volume? J Stroke Cerebrovasc Dis. 2014;23:2163-2168 


\section{Chapter 4}

\section{Alterations in Vascular Function Following Ischemic Stroke in Relation to Arginase 1 Expression}

Ashley B. Petrone, BS ${ }^{1,2}$

${ }^{1}$ Dept. of Neurobiology and Anatomy, and ${ }^{2}$ Center for Basic and

Translational Stroke Research

West Virginia University, One Medical Center Drive,

Morgantown WV 26506 
Background: Increased ARG1 expression within 24 hours of AIS symptom onset is positively correlated with increased stroke severity, as measured by the $\mathrm{NIH}$ stroke severity score (NIHSS). ARG1 expression has been shown to be associated and functional mediator of immune system dysregulation following AIS that may represent a functional mechanism that ARG1 may contribute to increased AIS severity; however, the role of ARG1 in mediating AIS severity may not be limited to immune system regulation. ARG1 can also mediate acute changes in vascular function through NO-dependent changes in arterial muscle tone. Carotid-femoral pulse wave velocity (cfPWV) is an assessment used to measure large-vessel arterial stiffness and is influenced by arterial smooth muscle tone. The aims of this study were to: (1) Characterize the changes in ARG1 expression that occur in response to AIS, (2) Characterize the changes in cfPWV that occur in response to AIS, and (3) To determine if ARG1 expression may be a functional mediator of vascular function following AIS. We hypothesize that increases in both $A R G 1$ expression and cfPWV will be associated with increased baseline stroke severity and poor outcomes. We also hypothesize that $A R G 1$ expression is correlated with cfPWV at both baseline and during AIS recovery.

Methods: Whole blood samples were drawn from AIS patients on three separate time points: baseline, 30 days, and 90 days after AIS. cIMT and cfPWV were also measured on three separate time points: baseline, 30 days, and 90 days following stroke.

Results: There is a strong unadjusted relationship between baseline ARG1 expression and baseline NIHSS. In mild AIS, cfPWV remains stable from baseline to 30 days poststroke. In contrast, cfPWV increased from baseline to 30 days post-stroke in severe AIS. Lastly, there appears to be a relationship between $\triangle A R G 1$ and $\triangle \mathrm{cfPWV}$ from baseline to 30 days post-stroke

Conclusions: Overall, we have described a novel relationship between ARG1 expression and cfPWV and AIS severity and recovery. ARG1 inhibition following AIS may represent a novel therapeutic strategy that may improve both immune and vascular function following AIS. 


\section{Introduction}

We have recently identified Arginase 1 (ARG1) as a marker of acute ischemic stroke (AIS) severity and outcome [1]. Specifically, increased ARG1 expression within 24 hours of AIS symptom onset is positively correlated with increased stroke severity, as measured by the NIH stroke severity score (NIHSS). Further, ARG1 expression has been shown to be associated and functional mediator of immune system dysregulation following AIS that may represent a functional mechanism that ARG1 may contribute to increased AIS severity; however, the role of ARG1 in mediating AIS severity may not be limited to immune system regulation. In addition to its role in regulating the immune response, ARG1 has been shown to affect vascular function by limiting the metabolism of L-arginine by nitric oxide synthases to nitric oxide (NO). This leads to decreased NO, resulting in changes in arterial structure, such as smooth muscle cell hypertrophy and increased collagen synthesis [2]. In addition to changes in arterial structure, ARG1 can also mediate acute changes in vascular function through NO-dependent changes in vasomechanics. Carotid-femoral pulse wave velocity (cfPWV) is an assessment used to measure largevessel arterial stiffness, and cfPWV is influenced by mean arterial pressure and arterial smooth muscle tone [3]. To this end, because of the role of NO in mediating vascular tone, acute changes in NO concentration can result in changes in cfPWV. Specifically, pharmacological NO inhibition has been shown to acutely increase cfPWV in human subjects [4]; therefore, given the role of ARG1 as an endogenous NO inhibitor, it is likely that increased ARG1 can also increase arterial stiffness (cfPWV) through a similar

mechanism; however, there have been no studies to date examining the relationship between ARG1 expression and cfPWV. Further, while increased cfPWV has been identified as a risk factor for AIS [5], the changes in cfPWV as a result of AIS and how 
these changes impact AIS recovery are unclear. The aims of this study were to: (1) Characterize the changes in ARG1 expression that occur in response to AIS, (2) Characterize the changes in cfPWV that occur in response to AIS, and (3) To determine if ARG1 expression may be a functional mediator of vascular function, specifically cIMT and cfPWV, following AIS. We hypothesize that increases in both ARG1 expression and cfPWV will be associated with increased baseline stroke severity and poor outcomes. We also hypothesize that ARG1 expression is correlated with cfPWV at both baseline and during AIS recovery.

\section{Methods}

\section{Subject Recruitment}

Informed consent was obtained from all individual participants included in the study. AIS patients and stroke-free control subjects were recruited from Ruby Memorial Hospital (Morgantown, WV). Male and female AIS patients were eligible for recruitment if the following inclusion criteria were met: (1) age $\geq 18$ years, (2) confirmation of acute stroke by neuroimaging (CT or MRI), and (3) had blood drawn within 24 hours of symptom onset/"last-known normal", prior to thrombolysis or interventional treatment. Patient data from the medical record were reviewed and recorded, including (1) National Institutes of Health Stroke Scale (NIHSS), (2) clinical laboratory analyses, including white blood cell differential, and (3) brain imaging (CT/MRI). Stroke-free control subjects were eligible for

recruitment if the following inclusion criteria were met: (1) age $\geq 18$ years, and (2) no history of AIS, transient ischemic attack, brain injury, or other overt central nervous system disease, and (3) recent hospitalization. Medical histories were obtained directly 
from stroke-free control subjects; however, complete access to medical records of control subjects was not available.

\section{Research Protocol Approval and Informed Consent}

This study received approval for human subject research from the institutional review boards of West Virginia University and Ruby Memorial Hospital (Morgantown, WV). Written informed consent was obtained from all subjects or their authorized representatives prior to performing study procedures.

\section{Study Design}

Whole blood samples were drawn from AIS patients on three separate time points: baseline (0-12 hours from symptom onset), 30 days, and 90 days after AIS. cIMT and cfPWV were also measured on three separate time points: baseline (24-48 hours of symptom onset, 30 days, and 90 days following stroke. For the 30 and 90 day time points, blood sample collection occurred immediately prior to CV assessments.

\section{Blood Sample Collection}

Peripheral venous whole blood was drawn from stroke subjects no later than 24 hours of stroke symptom onset. Blood was collected into PAXgene文 Blood RNA tubes (BectonDickinson). Immediately after blood collection, tubes were inverted 8-10 times and stored at $-80^{\circ} \mathrm{C}$ until analysis. 


\section{Gene Expression Analysis}

PAXgene文 Blood RNA tubes were thawed overnight (16-20 hrs) at room temperature prior to RNA extraction. The PAXgene Blood RNA kit (Pre-Analytix) was used to purify/extract intracellular RNA, per manufacturer's instructions. RNA concentration and quality was determined by absorbance using a Take3 Trio Microplate (BioTek夕) read on a Syntek Hybrid Plate Reader, and analyzed using Gen5 (BioTek) software. A260/A280 values between 1.8 and 2.2 were considered acceptable RNA quality.

RNA was converted to cDNA using the High-Capacity Reverse Transcription Kit (Applied Biosystems). cDNA (10 ng) was used for quantitative real-time PCR amplification using SYBR Green chemistry using the Rotor-Gene Q real-time PCR cycler (Qiagen). The following Quantitect primers were used: ARG1 (NM_000045,NM_001244438)and PPIB (NM_000942) (Qiagen). PPIB served as a stable, endogenous reference gene to normalize ARG1 expression.

\section{Arterial Function}

Pulse wave analysis was performed non-invasively on the right radial artery (SphygmoCor system, AtCor Medical, Sydney, NSW, Australia). From the radial artery pressure wave, the SphygmoCor system calculates a central pressure waveform that accurately replicates the ascending aortic pressure wave obtained from invasive measures. From the central wave, central blood pressure was also determined. Cf-PWV was obtained using the SpygmoCor XCEL PWV device (SphygmoCor system, AtCor Medical, Sydney, NSW, Australia). A cuff was placed around the femoral artery of the subject to capture the femoral waveform/pulse, and a tonometer pressure sensor was 
used to capture the carotid waveform/pulse. The pulse transit time is the time that the pulse takes to travel from the carotid to the femoral artery. The distance between the carotid and femoral arteries was measured for each subject, and the cfPWV automatically determined by dividing the distance by the pulse transit time.

\section{Arterial Geometry}

In the supine position, B-mode ultrasound (GE Vivid I; GE healthcare, Chalfont St Giles, UK) images were obtained of the right common carotid artery, $1-2 \mathrm{~cm}$ proximal to the carotid bifurcation to measure clMT.

\section{Statistical Analysis}

The relationships between ARG1 expression, NIHSS, cIMT, and cfPWV were tested using Spearman's rank-order correlations. Linear regression analysis was used to identify statistically significant relationships when controlling for confounding variables, specifically age, sex, hypertension, diabetes, dyslipidemia, and smoking.

\section{Results}

\section{Clinical Characteristics}

A total of 12 subjects participated in this study, and subjects' demographic information is summarized in Table 1. In addition, the mean baseline cIMT for the subject cohort was $0.59 \pm 0.08 \mathrm{~mm}($ mean $\pm S D)$, and the mean baseline cfPWV was $9 \pm 1 \mathrm{~m} / \mathrm{s}$ (mean $\pm S D$ ) 


\section{Relationship between ARG1 and Stroke Severity}

There is a strong unadjusted relationship between baseline ARG1 expression and baseline NIHSS ( $r=0.660, p=0.038)$ (Figure 1). After linear regression analysis controlling for age, sex, HTN, DM, dyslipidemia, and prior stroke, the relationship between baseline ARG1 expression and baseline NIHSS remained significant $(p=0.032)$. Further, there is a strong unadjusted relationship between 30 day ARG1 expression and baseline NIHSS $(r=0.766, p=0.01)$ (not shown). After linear regression analysis controlling for age, sex,

HTN, DM, dyslipidemia, prior stroke, and tPA treatment, the relationship between 30 day ARG1 expression and baseline NIHSS was no longer significant $(p=0.401)$.

\section{Change in ARG1 Expression 30 Days Post-Stroke}

While our limited sample size does not allow for meaningful statistical analysis, we observed several trends in changes in ARG1 expression 30 days post-stroke (Figure 2). As expected, we observed that in a single control subject $A R G 1$ expression remained stable from baseline to 30 days. Upon examination of $n=2$ mild AIS patients, we observe a decrease in ARG1 expression from baseline to 30 days. In contrast, we observed that in a single severe AIS patient, ARG1 expression increased from baseline to 30 days poststroke. In the moderate AIS group $(n=2)$, there were both increases and decreases in ARG1 expression from baseline to 30 days.

Change in cfPWV 90 Days Post-Stroke 
While our limited sample size does not allow for meaningful statistical analysis, we observed several trends in changes in cfPWV from baseline to 90 days post-stroke

(Figure 3). As expected, we observed that in a single control subject cfPWV remained stable from baseline to 30 days. Upon examination of $n=3$ mild AIS patients, we observe a decrease in cfPWV from baseline to 30 days (no 90 day data available). Further, in the mild group, cfPWV remains stable from 30 to 90 days post-stroke. In contrast, we observed that in a single severe AIS patient, cfPWV increased from baseline to 30 days post-stroke (no 90 day data available).

\section{Relationship between ARG1 and cfPWV}

While our limited sample size does not allow for meaningful statistical analysis, there appears to be a relationship between $\triangle A R G 1$ and $\triangle \mathrm{cfPWV}$ from baseline to 30 days poststroke (Figure 4). In a single control subject, we observe that neither cfPWV nor ARG1 expression changes from baseline to 30 day follow-up visit. In a single mild AIS patient, we observe that a large decrease in cfPWV is associated with a decrease in ARG1 expression. In contrast, in a single severe AIS patient, we observe that a large increase in cfPWV is associated with a increase in $A R G 1$ expression.

Relationship between ARG1 and cIMT

There was not a significant relationship between ARG1 and CIMT neither at baseline $(\mathrm{r}=0.098, p=0.817)$ nor at 30 days post-stroke $(r=0.083, p=0.167)$ (not shown). Further, there were no notable decreases in cIMT in any of the subjects across the 10-week lifestyle intervention, and subsequently, no correlation between $\triangle A R G 1$ and $\triangle \mathrm{clMT}$.

\section{Discussion}


This is the first study aimed to characterize the changes in $A R G 1$ expression and vascular function that occur in response to AIS. While this study is preliminary and evocative in nature, we have both confirmed relationships that had been described in previous studies and also provide novel evidence to support patterns of $A R G 1$ expression and changes in cfPWV that may be observed following AIS. First, we confirmed that baseline ARG1 is a marker of AIS severity, as measured by NIHSS. Specifically, increased baseline ARG1 is correlated with increased NIHSS. We have expanded this finding to describe how changes in ARG1 expression that occur following AIS relate to AIS recovery. We have shown that there are AIS severity-dependent changes in ARG1 expression that occur during recovery. Specifically, we have shown that not only is baseline ARG1 expression lower in mild AIS patients compared to severe, but also that ARG1 expression tends to decrease over a 30-day recovery period. In contrast, in severe AIS, ARG1 expression tends to decrease over a 30 -day recovery period. This marked difference in $\triangle A R G 1$ between mild and severe groups may represent a functional mechanism that may account for poor recovery seen in severe AIS patients. Further, this elevated ARG1 expression, at both baseline and 30 days into recovery in severe AIS patients, may contribute to poor recovery via mechanisms that are independent of the degree of brain damage suffered at baseline. We have previously shown that the neutrophil-lymphocyte ratio (NLR) is a marker of immune dysfunction following AIS that is associated with increased AIS severity and poor recovery. Specifically, an increased baseline NLR is associated with stroke severity and is predictive of AIS outcome, as measured by the Modified Rankin Scale $[1,6]$. Further, we have examined the change in NLR from baseline to 30 days post-AIS and reported that severe AIS patients have an overall increase in NLR from baseline to 
30 days, whereas mild AIS patients have a decrease or no change in NLR over the 30 day recovery period (Petrone, unpublished). We have also shown that baseline ARG1 expression is positively correlated with baseline NLR [1]. Combining the results of these previous studies and the current study, we may speculate that $A R G 1$ may be the mediator of NLR at both baseline and during recovery.

While we have established ample evidence to suggest that $A R G 1$ contributes to increased AIS severity and poor outcome following AIS by altering immune function, we hypothesized that the role of ARG1 in AIS was not limited to mediating the immune response, given its traditional role in mediating vascular function. In support of our hypothesis, we have established a relationship between ARG1 expression and cfPWV following AIS. Further, independent of ARG1 expression, we have characterized the changes in cfPWV that occur following AIS. In summary, severe AIS patients have an overall increase in cfPWV from baseline to 30 days, whereas mild AIS patients have a decrease or no change in cfPWV over the 30-day recovery period. While it is generally agreed, that increased cfPWV is indicative of poor CV health, future studies are needed to address the molecular mechanisms resulting in an increase in cfPWV following stroke, because it may be possible that increased cfPWV in response to AIS may be a physiological response to decrease AIS severity.

Overall, we have described a relationship between ARG1 expression and cfPWV during AIS recovery. ARG1 inhibition following AIS may represent a novel therapeutic strategy, administered both acutely and chronically following AIS, and the beneficial mechanism of action of ARG1 inhibition may target not only one, but several 
pathophysiological mechanisms following AIS, including both immune dysregulation and vascular dysfunction.

Table 1: Demographic Information for Study Participants $(n=12)$

\begin{tabular}{rc}
\hline Age, yrs, mean \pm SD & $58 \pm 16$ \\
Sex, $\mathbf{n}(\%$ female) & $7(58)$ \\
\hline Previous Ischemic Stroke (\%) & $3(25)$ \\
NIH Stroke Score, mean (range) & $5.8(0-18)$ \\
Received tPA (\%) & $4(33)$ \\
Hypertension (\%) & $10(83)$ \\
Diabetes (\%) & $4(33)$ \\
Dyslipidemia (\%) & $3(25)$ \\
\hline
\end{tabular}




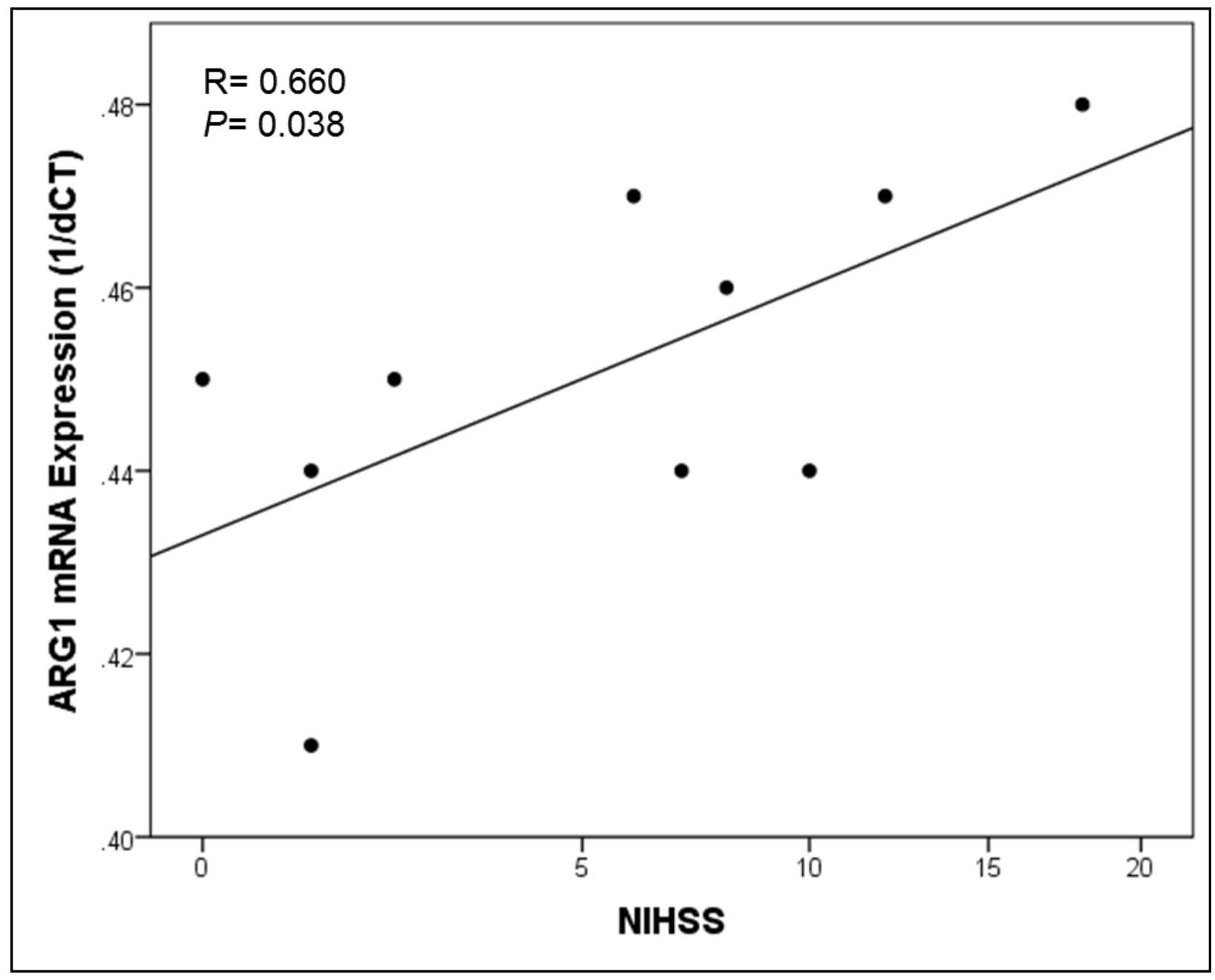


Figure 1: The Relationship between ARG1 Expression and AIS Severity. There is a strong unadjusted relationship between baseline ARG1 expression and baseline NIHSS $(r=0.660, p=0.038)$.

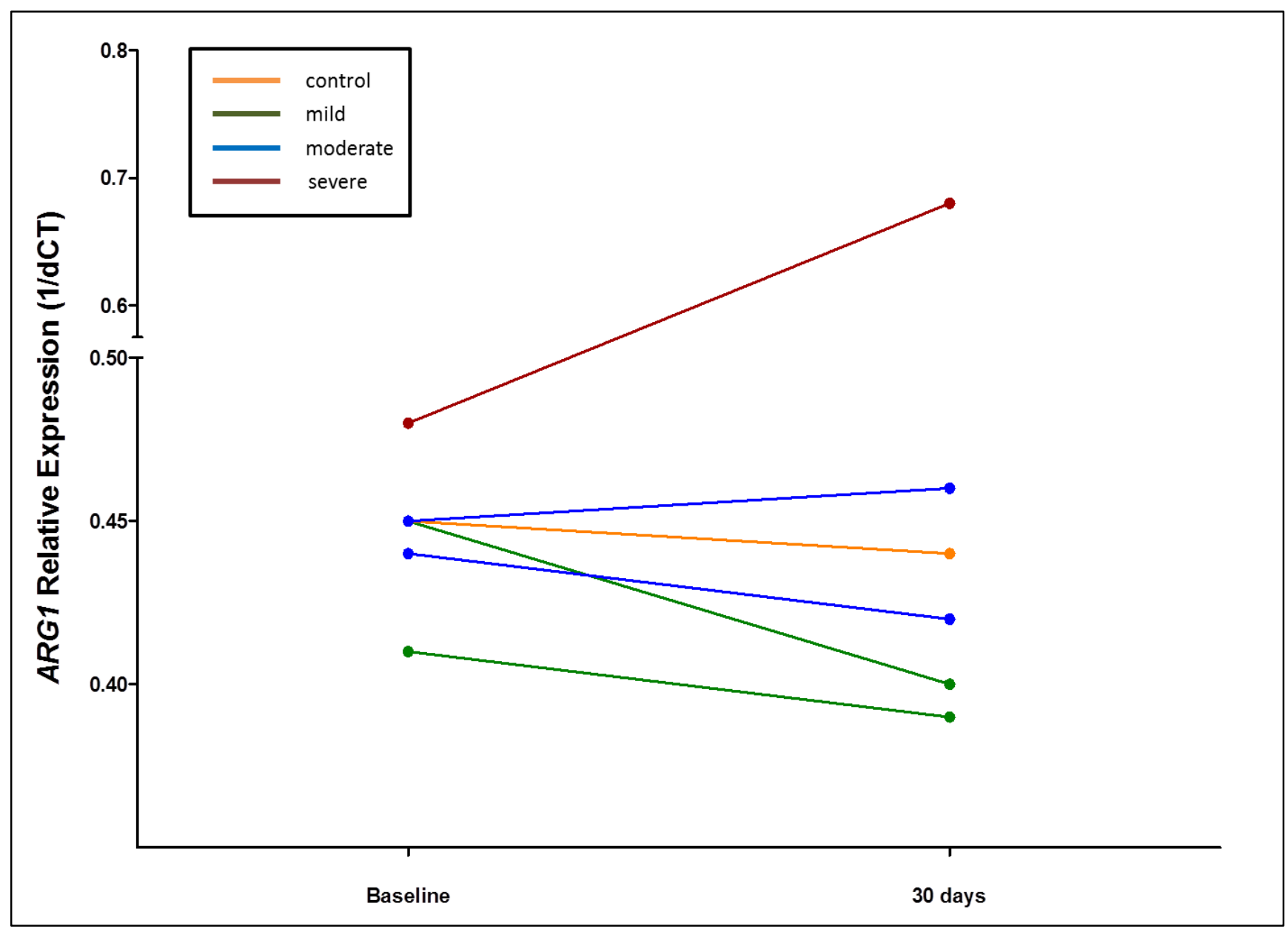

Figure 2: Changes in ARG1 Expression in AIS Recovery. In a single control subject ARG1 expression remained stable from baseline to 30 days (orange). In mild AIS 
patients, there is a decrease in ARG1 expression from baseline to 30 days (green). $A R G 1$ expression increased from baseline to 30 days post-stroke in severe AIS (red). In the moderate AIS group $(\mathrm{n}=2)$, there were both increases and decreases in ARG1 expression from baseline to 30 days (blue).

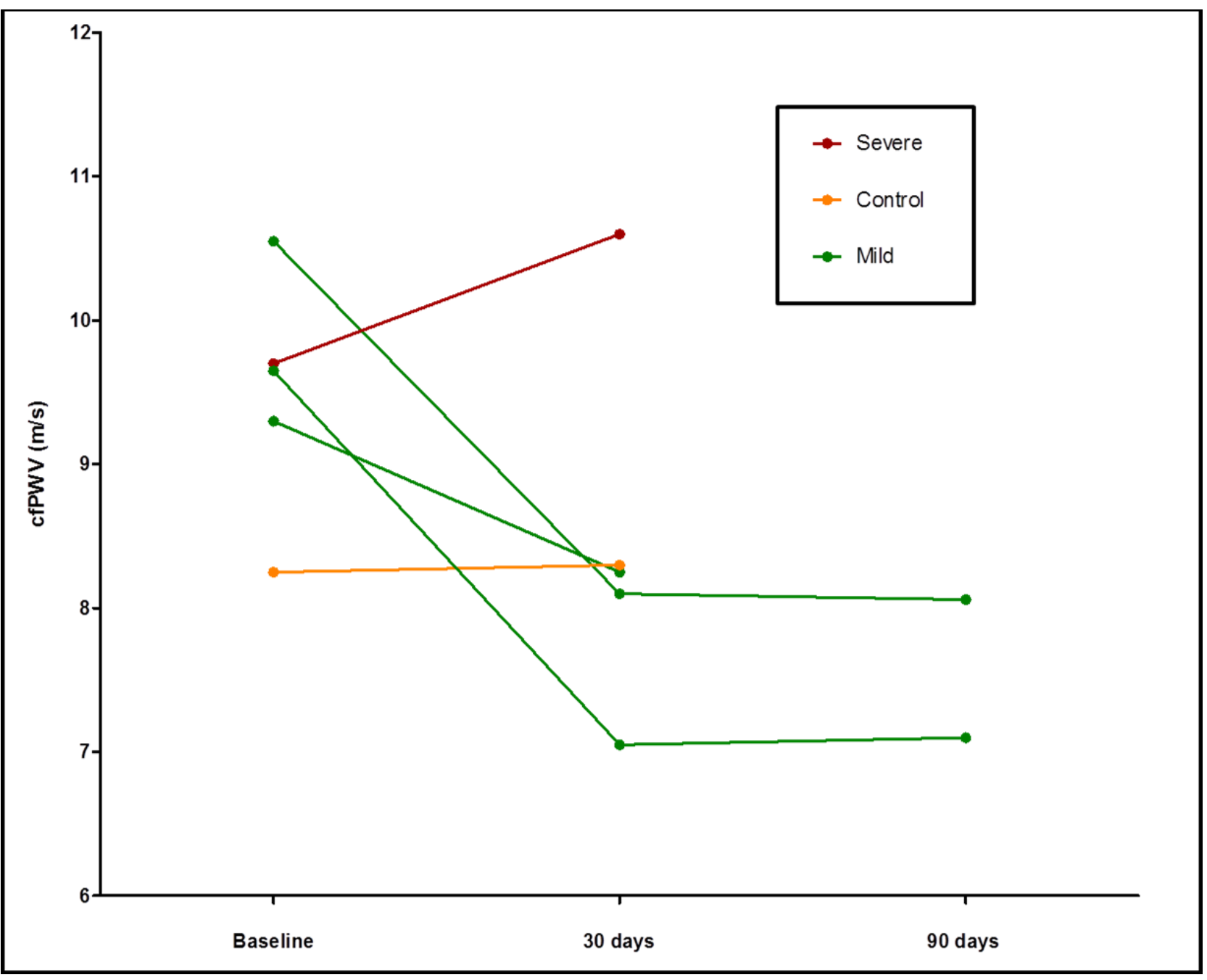

Figure 3: Changes in cfPWV in AIS Recovery. In a single control subject, cfPWV remained stable from baseline to 30 days (orange). In the mild AIS group, there is a decrease in cfPWV from baseline to 30 days (green). Further, in the mild group, cfPWV 
remains stable from 30 to 90 days post-stroke (green). In a single severe AIS patient, cfPWV increased from baseline to 30 days post-stroke (red).

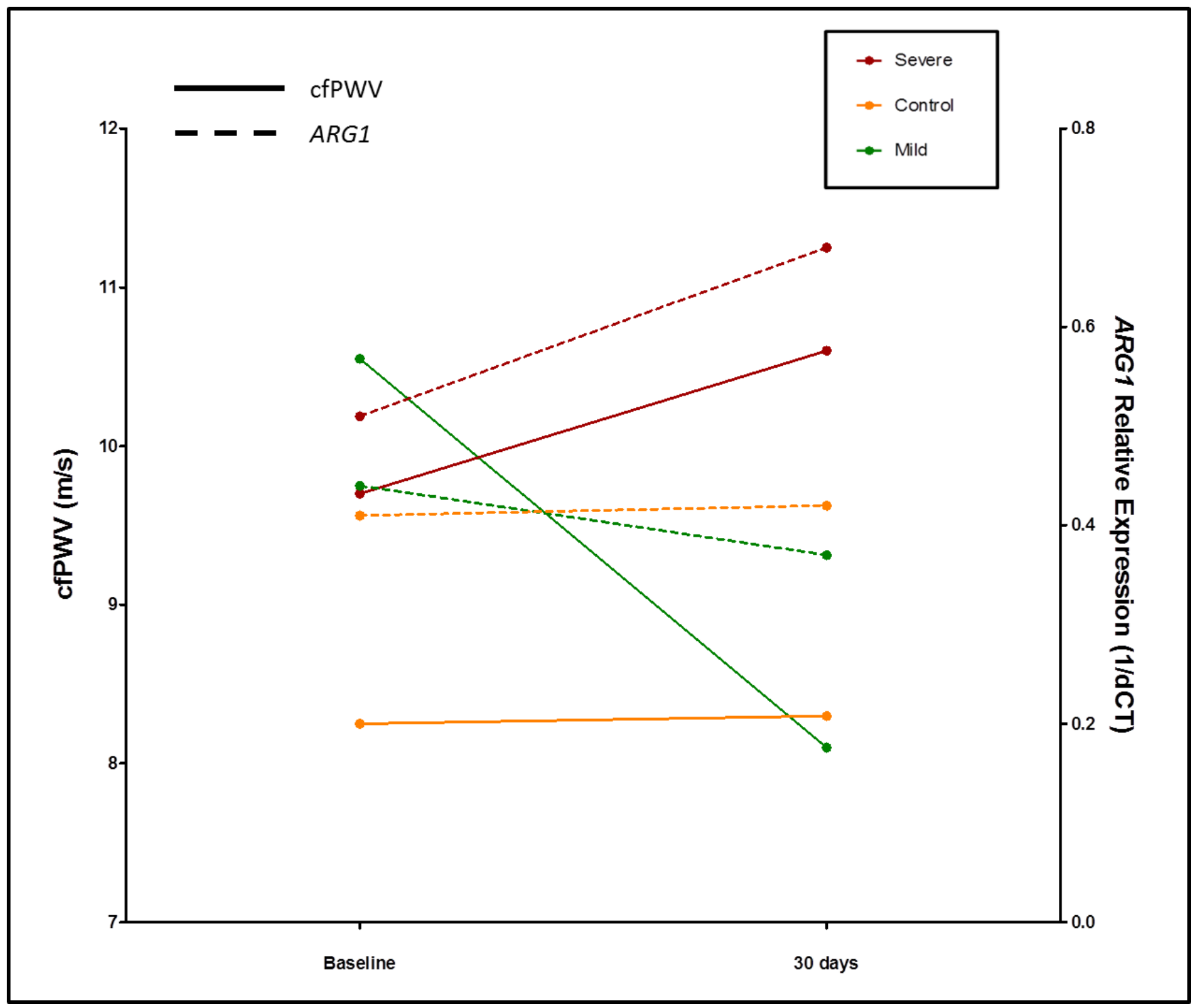

Figure 4: Relationship between ARG1 Expression and cfPWV in AIS Recovery. In a single control subject, neither cfPWV nor ARG1 expression changes from baseline to 30-day follow-up (orange). In a single mild AIS patient, there is a large decrease in cfPWV is associated with a decrease in $A R G 1$ expression (green). In a single severe 
AIS patient, there is large increase in cfPWV is associated with an increase in ARG1 expression (red).

\section{References}

1. Petrone AB, O'Connell GC, Regier MD, Chantler PD, Simpkins JW, Barr TL. The role of arginase 1 in post-stroke immunosuppression and ischemic stroke severity. Trans/ Stroke Res. 2015

2. Durante W, Johnson FK, Johnson RA. Arginase: A critical regulator of nitric oxide synthesis and vascular function. Clin Exp Pharmacol Physiol. 2007;34:906-911

3. Fok H, Jiang B, Clapp B, Chowienczyk P. Regulation of vascular tone and pulse wave velocity in human muscular conduit arteries. 2012

4. Stewart AD, Millasseau SC, Kearney MT, Ritter JM, Chowienczyk PJ. Effects of inhibition of basal nitric oxide synthesis on carotid-femoral pulse wave velocity and augmentation index in humans. Hypertension. 2003;42:915-918

5. Mattace-Raso FUS, Cammen TJMvd, Hofman A, Popele NMv, Bos ML, Schalekamp MADH, et al. Arterial stiffness and risk of coronary heart disease and stroke. 2006

6. Brooks SD, Spears C, Cummings C, VanGilder RL, Stinehart KR, Gutmann L, et al. Admission neutrophil-lymphocyte ratio predicts 90 day outcome after endovascular stroke therapy. J Neurointerv Surg. 2014;6:578-583 


\title{
Chapter 5
}

Evaluation of the Relationship Between ARG1 Expression and Cardiovascular Function Following Lifestyle Modification

\author{
Ashley B. Petrone, BS ${ }^{1,2}$ \\ ${ }^{1}$ Dept. of Neurobiology and Anatomy, and ${ }^{2}$ Center for Basic and \\ Translational Stroke Research \\ West Virginia University, One Medical Center Drive, \\ Morgantown WV 26506
}


Background: Arginase 1 (ARG1) had been established as a mediator of endothelial function. Specifically, ARG1 limits the metabolism of L-arginine by both iNOS and eNOS to NO, resulting in changes in arterial structure, such as smooth muscle cell hypertrophy and increased collagen synthesis. We have previously characterized the changes in carotid-intima medial thickness (cIMT) and carotid-femoral pulse wave velocity (cfPWV) that occur post-stroke; however, because stroke recovery is generally associated with treatment and improvement of CV risk factors, such as hypertension and diabetes, it is difficult to distinguish the changes in cIMT and cfPWV that are a direct response to stroke from those that result from overall CV health improvements. The goal of this study was to characterize the changes in ARG1 expression, as well as cIMT and cfPWV, following a 10-week lifestyle modification, and we hypothesize that decreases in CV risk factors will correlate with decreases in ARG1 expression.

Methods: CVD subjects were assessed at beginning of the study and following a 10week lifestyle modification program designed to reduce CV risk factors. Body composition, functional exercise capacity, and vascular assessments (cIMT and cfPWV) were performed at each time point. Blood samples were also collected to quantify ARG1 expression.

Results: There was an unadjusted positive correlation between baseline ARG1 expression and both cIMT $(r=0.844, p=0.001)$ and cfPWV $(r=0.743, p=0.006)$. There were no substantial changes in cIMT across the 10-week intervention; however, several subjects in the cohort had substantial changes in cfPWV ( $\triangle \mathrm{cfPWV}$ ) and ARG1 ( $\triangle A R G 1)$. Further, there was an unadjusted positive correlation between $\triangle \mathrm{cfPWV}$ and $\triangle A R G 1$ $(r=0.703, p=0.016)$.

Conclusions: This is the first report of a relationship between ARG1, cfPWV, and cIMT in subjects with $\mathrm{CV}$ risk factors. While this preliminary analysis requires validation to confirm the relationships described herein, it appears as that ARG1 may be a mediator of cfPWV. With the ultimate goal of developing an ARG1 inhibitor for therapeutic use, this study provides support for the hypothesis that ARG1 inhibition may be used as a proactive therapeutic to improve CV health. 


\section{Introduction}

We have recently identified Arginase 1 (ARG1) as a marker of immune dysfunction following ischemic stroke [1). Further, ARG1 may mediate changes in immune function, as well as other physiological processes, that contribute to increased risk of stroke and poor recovery. Prior to the association between ARG1 and immune function, ARG1 had been established as a mediator of endothelial function [2]. Specifically, ARG1 limits the metabolism of L-arginine by both iNOS and eNOS to NO, which leads to decreased NO, resulting in changes in arterial structure, such as smooth muscle cell hypertrophy and increased collagen synthesis [2]. These changes in structure result in medial wall thickening and arterial stiffness. Arterial stiffness has been identified as a risk factor for several cardiovascular diseases, including ischemic stroke, thus strategies to reduce arterial stiffness and prevent abhorrent vascular remodeling, may serve as a proactive therapeutic to reduce the risk of stroke [3]. We have previously characterized the changes in carotid-intima medial thickness (cIMT) and carotid-femoral pulse wave velocity (cfPWV) that occur post-stroke [Petrone unpublished, chapter 3]; however, because stroke recovery is generally associated with treatment and improvement of CV risk factors, such as hypertension and diabetes, it is difficult to distinguish the changes in cIMT and cfPWV that are a direct response to stroke from those that result from overall CV health improvements. Further, we described the relationship between changes in ARG1 expression that occur along with changes in cIMT and cfPWV following stroke, but similarly, the changes in $A R G 1$ expression that are a direct function of stroke are difficult to distinguish from changes in CV function. There is a large body of evidence that suggests that current CV risk management therapeutics, such as statins, inhibit arginase 
activity, and in addition to their primary target effect, some of the efficacy of these drugs may be the result of the off-target arginase inhibition [4]. To this end, a specific-ARG1 inhibitor may be a potential therapeutic strategy in CV disease, stroke, and likely several other conditions; however, more information is needed to understand the functional role of ARG1 and the patterns of expression that occur over time in response to various conditions. The goal of this study was to characterize the changes in ARG1 expression, as well as cIMT and cfPWV, following a 10-week lifestyle modification. This 10-week intervention was designed to improve $\mathrm{CV}$ health, in a similar fashion and timeline that we assessed in ischemic stroke patients (Petrone, unpublished Chapter 3). We hypothesize that decreases in CV risk factors, such as CIMT, cfPWV, blood pressure, and body weight, will correlate with decreases in ARG1 expression.

\section{Methods}

\section{Subject Recruitment}

Informed written consent was obtained from all subjects prior to study participation. Subjects were eligible for study participation if diagnosed with CVD, as defined by the criteria listed in Table 1.

\section{Study Design}

The rural Health/Heart Accelerating Research Transition (rHEART) was developed to promote healthy lifestyle changes in a rural population. The key feature of this study was to engage the subjects' family members or friends to participate with the subject to promote healthy lifestyle changes through accountability in the subject's home 
environment. Each subject had the following samples/measurements taken before and following a 10-week lifestyle intervention that included diet modification and weekly sessions to educate subjects on CVD health, including smoking cessation, stress management, physical activity, etc.

\section{Body Composition and Physical Performance}

Anthropometric assessments, including height and weight, were used to measure body mass index (BMI), as a measure of obesity $(\mathrm{BMI}>30)$. The 6 -minute walk test $(6 \mathrm{MWT})$ was used to compare functional exercise capacity. The subjects were asked to walk back and forth over a 30-meter distance, and the distance walked in 6 minutes was recorded. The 6MWT is inexpensive and easy to administer in a variety of locations, and is also less strenuous than a typical cardiac stress test, but still able to yield accurate information regarding functional exercise capacity [5].

\section{Assessments}

All CV assessments were obtained when subjects were fasted for at least 12 hours and after a minimum of 15 minutes quiet rest. Subjects were also asked to abstain from alcohol, caffeine, vitamins, and any other health supplement 24 hours prior to assessments. Further, CV assessments were obtained at the same time of the day both prior to and following the 10-week intervention.

\section{Arterial Function}


Pulse wave analysis was performed non-invasively on the right radial artery (SphygmoCor system, AtCor Medical, Sydney, NSW, Australia). From the radial artery pressure wave, the SphygmoCor system calculates a central pressure waveform that accurately replicates the ascending aortic pressure wave obtained from invasive measures. From the central wave, central blood pressure was also determined. Cf-PWV was obtained using the SpygmoCor XCEL PWV device (SphygmoCor system, AtCor Medical, Sydney, NSW, Australia). A cuff was placed around the femoral artery of the subject to capture the femoral waveform/pulse, and a tonometer pressure sensor was used to capture the carotid waveform/pulse. The pulse transit time is the time that the pulse takes to travel from the carotid to the femoral artery. The distance between the carotid and femoral arteries was measured for each subject, and the cfPWV automatically determined by dividing the distance by the pulse transit time.

\section{Arterial Geometry}

In the supine position, B-mode ultrasound (GE Vivid I; GE healthcare, Chalfont St Giles, UK) images were obtained of the right common carotid artery, $1-2 \mathrm{~cm}$ proximal to the carotid bifurcation to measure cIMT.

\section{Blood Sample Collection}

Venous blood was drawn to perform a metabolic panel to identify total cholesterol, highdensity lipoprotein (HDL), triglycerides, hemoglobin, and glucose levels following a 12 hour fast. Further, subjects' medical history was recorded for age, sex, smoking status, diabetes, hypertension, and other relevant health history. In addition to the metabolic 
panel, a second blood sample was collected into PAXgene女 Blood RNA tubes (BectonDickinson) for gene expression analysis. Immediately after blood collection, PAXgene女 tubes were inverted $8-10$ times and stored at $-80^{\circ} \mathrm{C}$ until analysis.

\section{Gene Expression Analysis}

PAXgene文 Blood RNA tubes were thawed overnight (16-20 hrs) at room temperature prior to RNA extraction. The PAXgene Blood RNA kit (Pre-Analytix) was used to purify/extract intracellular RNA, per manufacturer's instructions. RNA concentration and quality was determined by absorbance using a Take3 Trio Microplate (BioTek夕) read on a Syntek Hybrid Plate Reader, and analyzed using Gen5 (BioTek) software. A260/A280 values between 1.8 and 2.2 were considered acceptable RNA quality.

RNA was converted to cDNA using the High-Capacity Reverse Transcription Kit (Applied Biosystems). cDNA (10 ng) was used for quantitative real-time PCR amplification using SYBR Green chemistry using the Rotor-Gene Q real-time PCR cycler (Qiagen). The following Quantitect primers were used: ARG1 (NM_000045,NM_001244438)and PPIB (NM_000942) (Qiagen). PPIB served as a stable, endogenous reference gene to normalize $A R G 1$ expression.

\section{Statistical Analysis}

The relationships between ARG1 expression, cIMT, and cfPWV were tested using Spearman's rank-order correlations. Linear regression analysis was used to identify statstically significant relationships when controlling for confounding variables, specifically age, sex, hypertension, diabetes, dyslipidemia, and smoking. 


\section{Results}

\section{Clinical Characteristics}

A total of 12 subjects participated in this study, and subjects' demographic information is summarized in Table 2 . The subject cohort was predominantly female $(92 \%)$ and the subjects' ages ranged from 20-76 years. The mean pre-intervention cIMT for the subject cohort was $0.65 \pm 0.17 \mathrm{~mm}$ (mean $\pm \mathrm{SD}$ ), and the mean pre-intervention cfPWV was $8 \pm$ $1.7 \mathrm{~m} / \mathrm{s}($ mean $\pm \mathrm{SD})$.

\section{Relationship between cIMT and cfPWV}

There is a significant unadjusted relationship between cIMT and cfPWV ( $r=0.758$, $p=0.004$ ) (Figure 1). After linear regression analysis controlling for age, sex, HTN, DM, dyslipidemia, and smoking, the relationship between cIMT and cfPWV is not significantly significant $(p=0.405)$. However, both cIMT $(r=0.853, p=0.000)$ and cfPWV $(r=0.797$, $p=0.000$ ) are strongly correlated with age, and when age is not included as an independent variable in linear regression analysis, the relationship between cIMT and cfPWV is statistically significant controlling for the remaining confounders $(p=0.018)$.

\section{Relationship between ARG1 and CV Assessments}

There is an unadjusted positive correlation between $A R G 1$ mRNA expression and both $\operatorname{clMT}(r=0.844, p=0.001)$ (Figure 2) and cfPWV ( $r=0.743, p=0.006)$ (Figure 3). After linear regression analysis controlling for age, sex, HTN, DM, dyslipidemia, and smoking, the relationship between $A R G 1$ and cIMT remains weakly significant $(p=0.18)$. However, 
upon removal of age from regression analysis, the relationship between $A R G 1$ and cIMT is statistically significant controlling for the remaining confounders $(p=0.019)$. Similarly, after linear regression analysis controlling for age, sex, HTN, DM, dyslipidemia, and smoking, the relationship between $A R G 1$ and cfPWV is not statistically significant $(p=0.526)$. However, upon removal of age from regression analysis, the relationship between ARG1 and cfPWV is statistically significant controlling for the remaining confounders $(p=0.054)$.

There were no substantial changes in clMT across the 10-week intervention; however, several subjects in the cohort had substantial changes in cfPWV ( $\triangle \mathrm{cfPWV})$ and $A R G 1$ ( $\triangle A R G 1)$. Further, there was an unadjusted positive correlation between $\triangle \mathrm{cfPWV}$ and $\triangle A R G 1(r=0.703, p=0.016)$ (Figure 4); however, after linear regression analysis controlling for age, sex, HTN, DM, dyslipidemia, and smoking, the relationship between $\triangle A R G 1$ and $\triangle \mathrm{cfPWV}$ is not statistically significant ( $p=0.444)$, nor when removing age from the regression analysis $(p=0.244)$.

\section{Discussion}

To our knowledge, this is one of the only studies to directly characterize the relationship between ARG1 expression, cIMT, and cfPWV. Further, this is the only study to assess changes in ARG1 expression following a 10-week lifestyle modification program. First, similar to previous reports, we confirmed the strong relationship between cIMT and cfPWV. While cIMT is typically used to assess arterial geometry, and cfPWV is used to measure arterial stiffness, both measures are considered gold-standard techniques to assess overall vascular function. Because cfPWV has been shown to change more 
acutely than cIMT, we expected to see very little change in cIMT over the 10-week intervention, but we hypothesized that decreases in CV risk factors over the 10-week intervention would correlate with decreases in cfPWV. There were no associations between decreased body weight, cholesterol, triglycerides, increased functional exercise capacity, and cfPWV. However, in support of our primary hypothesis, we did observe a correlation between the 10-week $\triangle$ cfPWV and $\triangle A R G 1$ expression $(r=0.703, p=0.016)$, indicating that ARG1 may be a functional mediator of cfPWV.

There are several limitations of this study that need to be addressed. First, the sample size included in this study is extremely small $(n=12)$ and the results presented here should be interpreted as preliminary and evocative in nature. While there were strong, unadjusted relationships between ARG1 expression, cIMT, and cfPWV, these relationships were not significant when adjusting for confounding regressors. Specifically, each of the variables in question, ARG1, cfPWV, and cIMT have a strong positive correlation with increasing age, such that the relationships may be accounted for solely by age, rather than a representation of a functional relationship between the variables. To address this possibility, a larger sample size will be required in future studies. Further, a subject cohort with a limited age range may be used to reduce the impact of age in order to determine the true functional relationships between $A R G 1, \mathrm{cIMT}$, and cfPWV. The second limitation of this study is that the 10-week lifestyle modification produced very modest improvements in overall CV function, as measured by the metrics in this study. Despite this, we did observe a relationship between $\triangle \mathrm{cfPWV}$ and $\triangle A R G 1$ expression, where decreases in cfPWV were correlated with decreased ARG1 expression; however, 
a longer intervention or an intervention that leads to greater improvements in CV health may provide better evidence for a role of ARG1 in mediating these improvements.

In summary, this is the first report of a relationship between ARG1, cfPWV, and CIMT in subjects with CV risk factors. While this preliminary analysis requires validation to confirm the relationships described herein, it appears as that $A R G 1$ may be a mediator of cfPWV. With the ultimate goal of developing an ARG1 inhibitor for therapeutic use in ischemic stroke, this study provides support for the hypothesis that ARG1 inhibition, administered in the months following stroke, may improve stroke outcomes, and ARG1 inhibition may be used as a proactive therapeutic to improve CV health. 


\section{Table 1: Study Inclusion Criteria}

At least one of the following criteria were met to be defined as having CVD and to be eligible for study participation:

(1) Prior Myocardial Infarction (>2 months)

(2) Revascularization Procedure for Coronary Artery Disease ( $>2$ months)

(3) Heart Disease

(4) Stroke (>2 months)

(5) Type II Diabetes

(6) Hypertension - BP $>140 / 90$ or taking antihypertensive medication

(7) Obesity (BMI $\geq 30)$ 


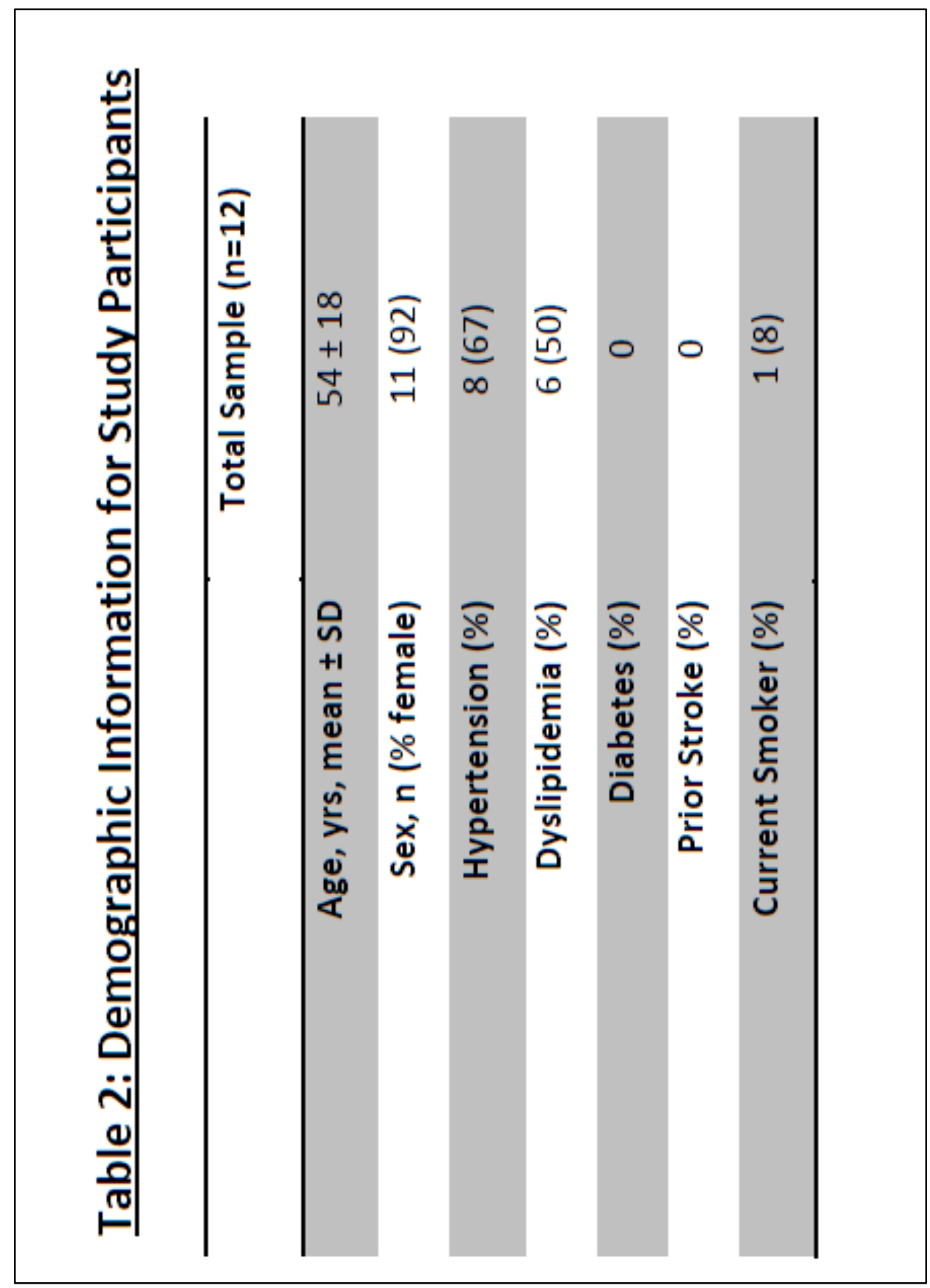




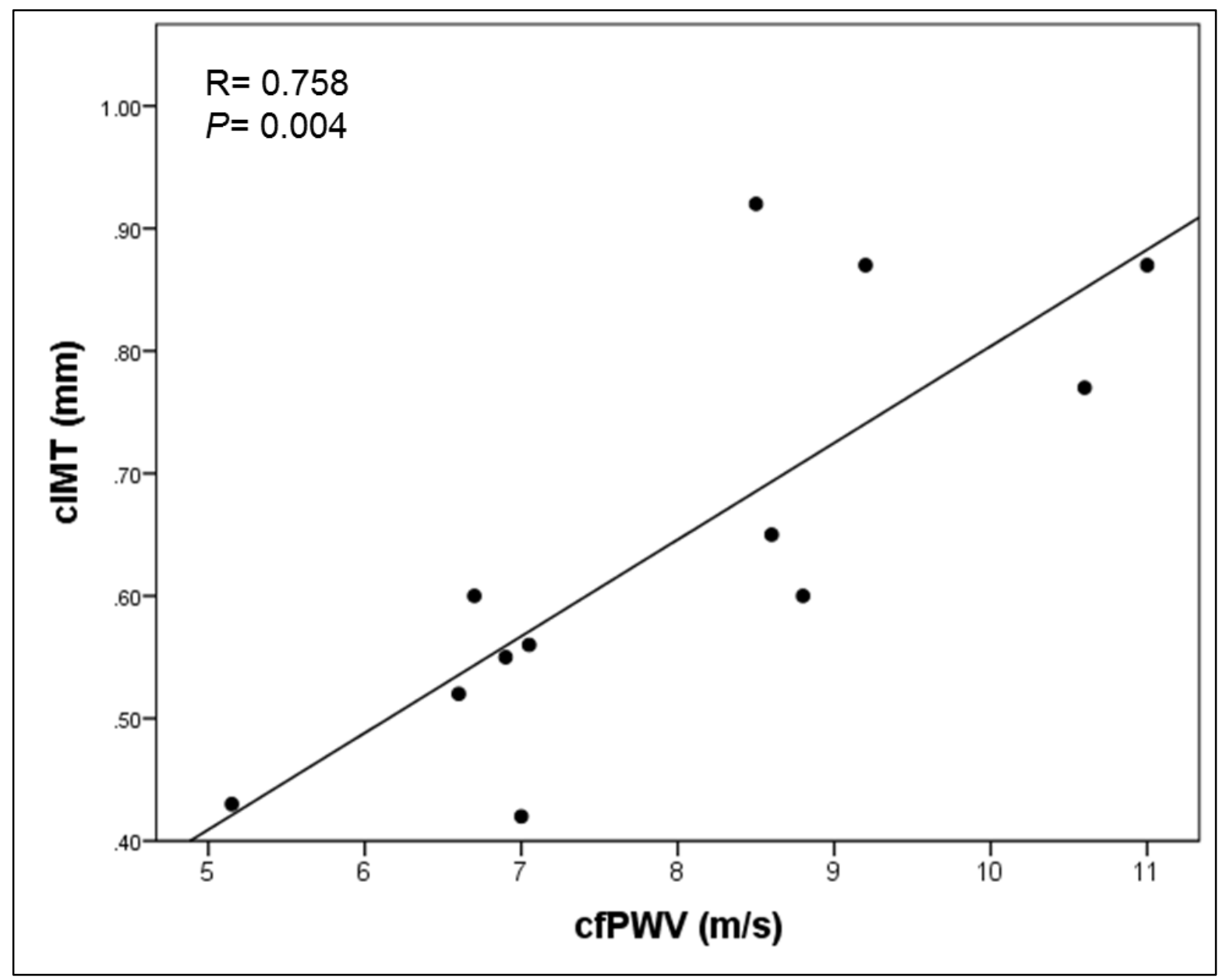

Figure 1. Relationship between cIMT and cfPWV. There is a significant unadjusted relationship between cIMT and cfPWV $(r=0.758, p=0.004)$. 


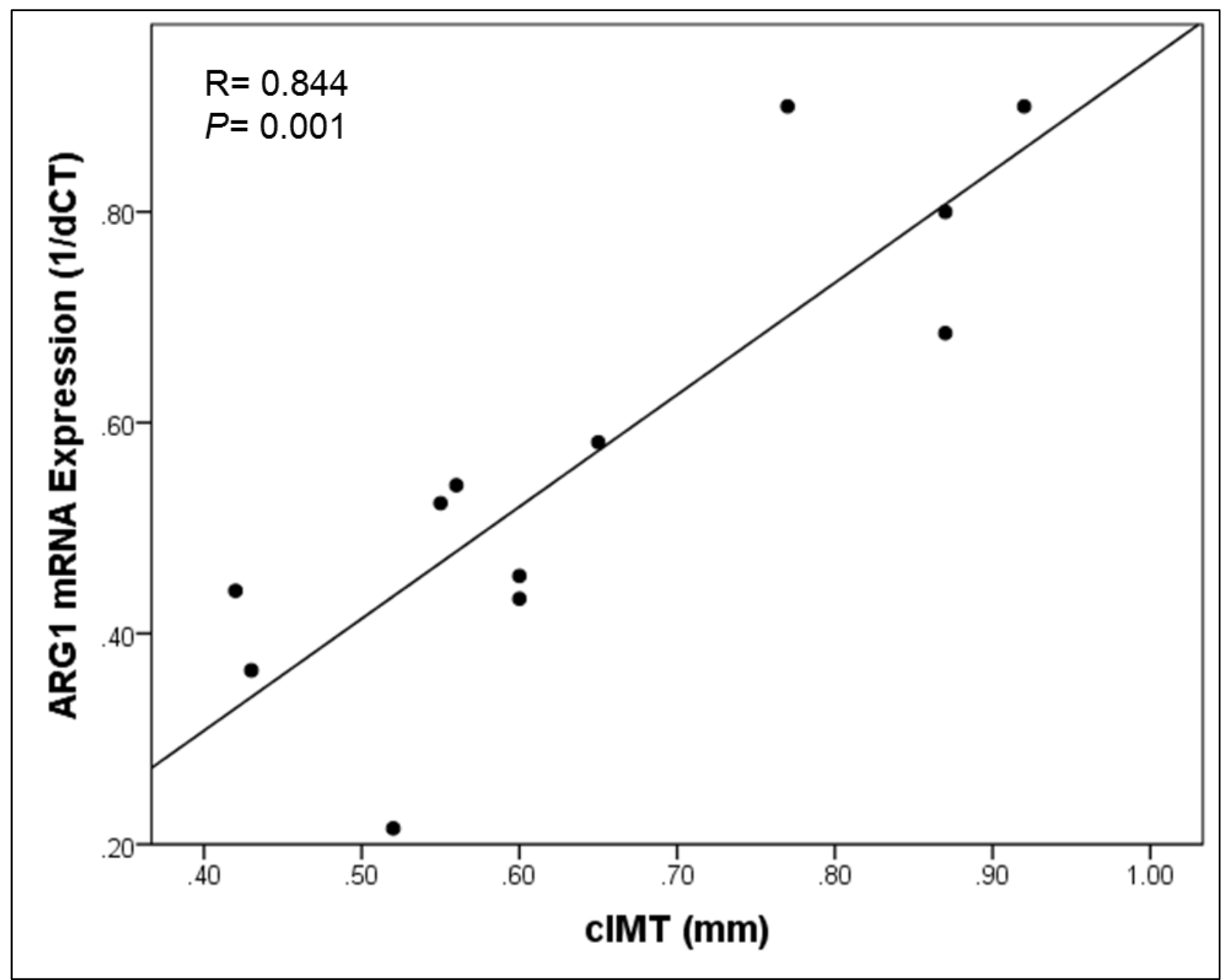

Figure 2. Relationship between cIMT and ARG1 Expression. There is an unadjusted positive correlation between $A R G 1$ mRNA expression and cIMT $(r=0.844, p=0.001)$. 


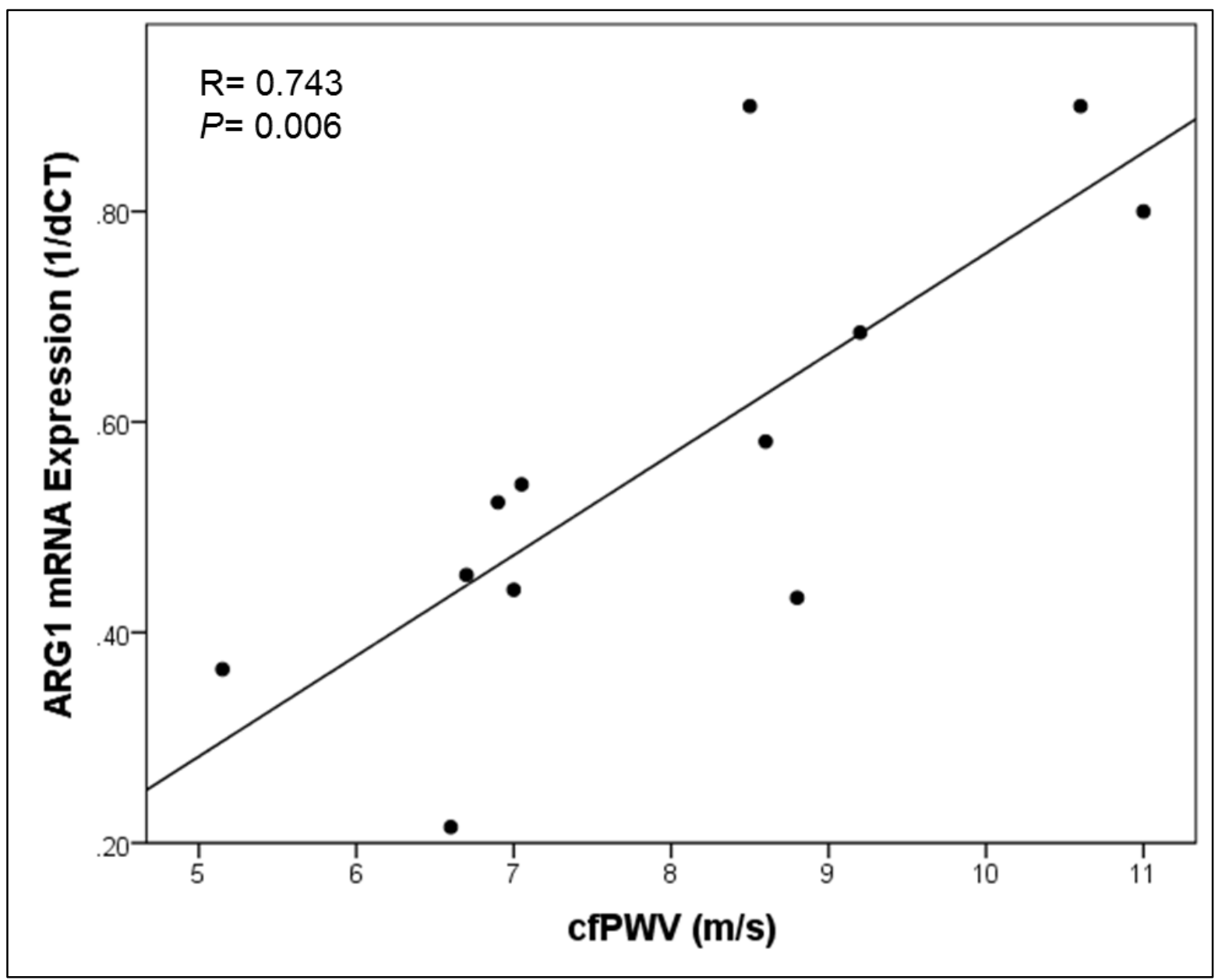

Figure 3. Relationship between cfPWV and ARG1 Expression. There is an unadjusted positive correlation between ARG1 mRNA expression and cfPWV $(r=0.743, p=0.006)$. 


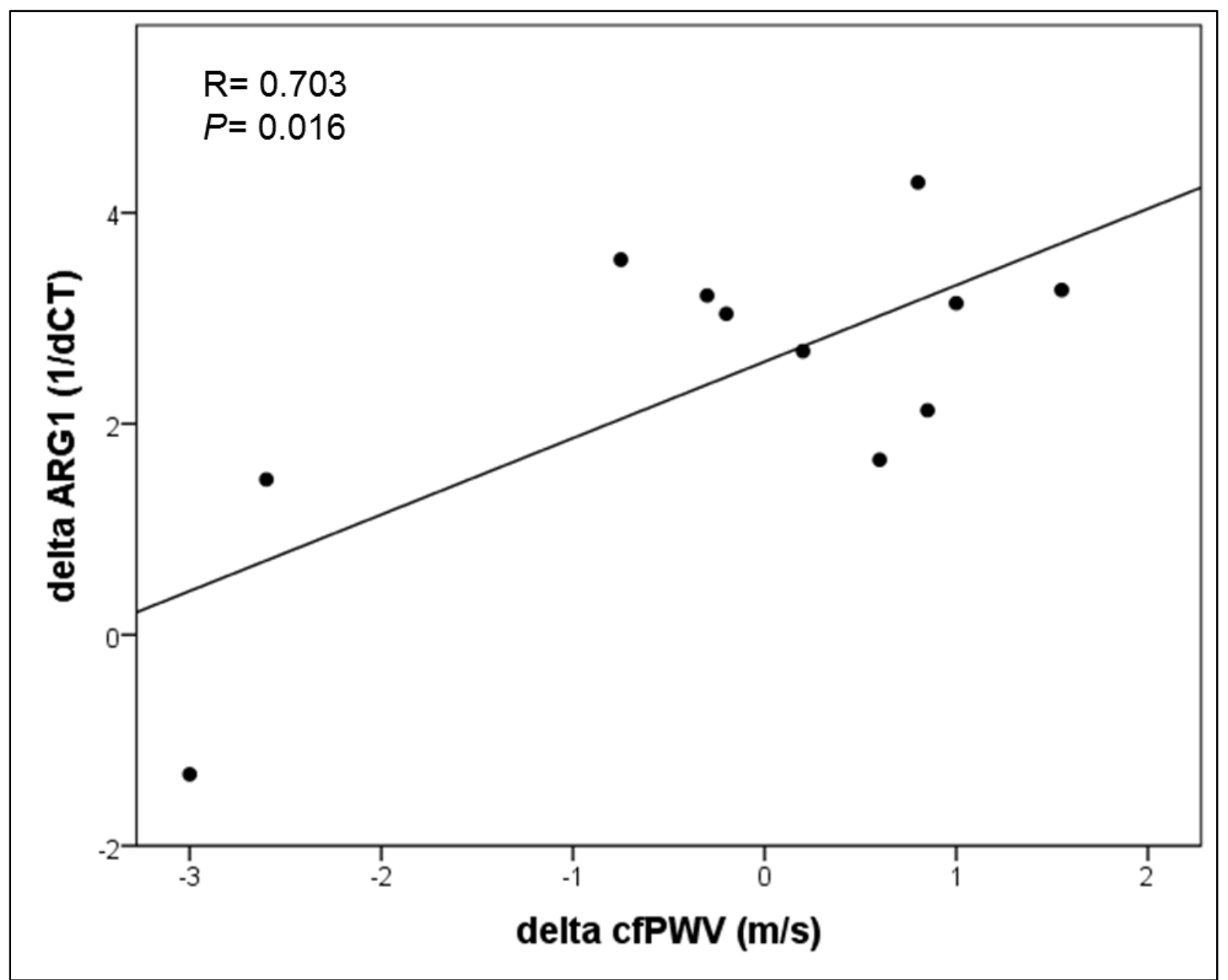

Figure 4. Relationship between $\triangle \mathrm{cfPWV}$ and $\triangle A R G 1$ Expression. There is an unadjusted positive correlation between $\triangle \mathrm{cfPWV}$ and $\triangle A R G 1(\mathrm{r}=0.703, p=0.016)$. 


\section{References}

1. Petrone AB, O'Connell GC, Regier MD, Chantler PD, Simpkins JW, Barr TL. The role of arginase 1 in post-stroke immunosuppression and ischemic stroke severity. Trans/ Stroke Res. 2015

2. Mattace-Raso FUS, Cammen TJMvd, Hofman A, Popele NMv, Bos ML, Schalekamp MADH, et al. Arterial stiffness and risk of coronary heart disease and stroke. 2006

3. Durante W. Role of arginase in vessel wall remodeling. Front Immunol. 2013;4

4. Durante W, Johnson FK, Johnson RA. Arginase: A critical regulator of nitric oxide synthesis and vascular function. Clin Exp Pharmacol Physiol. 2007;34:906-911

5. Bellet RN, Adams L, Morris NR. The 6-minute walk test in outpatient cardiac rehabilitation: Validity, reliability and responsiveness--a systematic review. Physiotherapy. 2012;98:277-286 


\title{
Chapter 6
}

Determination of the Cellular Origin of ARG1 Protein in Ischemic Stroke

\author{
Ashley B. Petrone, BS ${ }^{1,2}$
}

${ }^{1}$ Dept. of Neurobiology and Anatomy, and ${ }^{2}$ Center for Basic and

Translational Stroke Research

West Virginia University, One Medical Center Drive,

Morgantown WV 26506 
Background: There have been several studies evaluating the changes in gene expression in whole blood in response to AIS, and several markers have been identified by multiple studies. Of particular interest is the gene Arginase 1 (ARG1) that has been reported to be roughly 3-fold higher in the whole blood of AIS patients within 24 hours of AIS compared to healthy control subjects. We have recently shown that increased whole blood ARG1 expression is associated with increased AIS severity, immunosuppression, and poor outcome following AIS. In human control subjects, ARG1 expression is highest in neutrophils compared to other peripheral blood leukocytes. Despite this finding, the role and cellular origin of ARG1 protein in AIS remains to be determined. Thus our hypothesis was two-fold; first, ARG1 protein levels would be higher in the neutrophils of AIS patients compared to controls. Second, that within the AIS group, neutrophil ARG1 protein levels would be higher in severe AIS compared to mild.

Methods: Peripheral venous whole blood was drawn from controls and from stroke subjects no later than 24 hours of stroke symptom onset. Following RBC lysis, leukocytes were pelleted and fixed. Fixed leukocytes were isolated and intracellular ARG1 was stained and measured by Flow Cytometry. Mann-Whitney $U$ Tests were used to compare intracellular ARG1 between AIS and control groups and also between mild and severe AIS groups.

Results: Intracellular ARG1 was detected in both the neutrophil and monocyte fractions in both AIS and control patients; however, neither neutrophil nor monocyte ARG1 levels significantly differed between AIS and control. Further, in both AIS and control, ARG1 was detected at a higher level in the neutrophil fraction compared to the monocyte fraction. Within the AIS group, neutrophil ARG1 was significantly higher in the severe group compared to the mild AIS group $(p=0.008)$

Conclusions: Overall, the results of this study suggest that ARG1 protein expression is highest in neutrophils compared to other leukocyte populations. Neutrophil ARG1 may also increase in response to brain damage in severe AIS. These findings suggest that inhibition of systemic ARG1 protein may improve AIS outcome, and neutrophils may be a therapeutic target for ARG1 inhibition.

\section{Introduction}


The immune system plays a vital role in acute ischemic stroke (AIS) incidence, pathophysiology, and recovery $[1,2]$. While an immune response is critical to resolution of ischemic damage in the brain, these alterations in immune function can affect systemic immune function outside of the brain. There have been a large number of studies evaluating the changes in inflammatory markers, immune cell profiles, and other measures of immune function in both the peripheral blood and brain following AIS. While a complete understanding of all of the alterations in immune function in response to AIS are remains unknown, it is generally agreed upon that there is an acute increase in proinflammatory cytokines, such as IL-6 and TNFa, in both the brain and periphery $[2,3]$. Despite this rapid increase in inflammation, there is also a degree of immune suppression that occurs systemically, termed post-stroke immunosuppression. Post-stroke immunosuppression is characterized by increased anti-inflammatory cytokines, lymphopenia, and splenic atrophy [4]; however, the molecular mechanisms giving rise to this clinical phenomenon are unknown. There have been several studies evaluating the changes in gene expression in whole blood in response to AIS, and although independent investigators performed these studies, several markers have been identified by multiple studies. Of particular interest is the gene Arginase 1 ( $A R G 1)$ that has been reported to be roughly 3-fold higher in the whole blood of AIS patients within 24 hours of AIS compared to healthy control subjects [5-7]. We have also recently shown that increased whole blood ARG1 expression is associated with increased AIS severity, immunosuppression, and poor outcome following AIS [6]. In humans, ARG1 expression is highest in neutrophils compared to other peripheral blood leukocytes [7], and ARG1 protein released from neutrophils suppresses $\mathrm{T}$ lymphocyte proliferation through downregulation of $\mathrm{T}$ 
lymphocyte $\operatorname{CD} 3 \zeta$ chain [8]. Despite this, the role and cellular origin of ARG1 protein in AIS remains to be determined. The aims of this study were to expand upon previous studies to (1) Determine whether ARG1 protein, in addition to mRNA expression, is highest within neutrophils compared to other leukocyte fractions, (2) Determine if neutrophil ARG1 protein levels differ between AIS and control and (3) Characterize the change in neutrophil ARG1 protein that may occur with increasing AIS severity. We hypothesize that ARG1 protein will be highest in neutrophils compared to other leukocyte populations in both AIS and control; however, ARG1 protein levels will be higher in the neutrophils of AIS patients compared to controls. Further, we hypothesize that within the AIS group, neutrophil ARG1 protein levels will be higher in severe AIS compared to mild.

\section{Methods}

\section{Subject Recruitment}

Informed consent was obtained from all individual participants included in the study. AIS patients and stroke-free control subjects were recruited from Ruby Memorial Hospital (Morgantown, WV). Male and female AIS patients were eligible for recruitment if the following inclusion criteria were met: (1) age $\geq 18$ years, (2) confirmation of acute stroke by neuroimaging (CT or MRI), and (3) had blood drawn within 24 hours of symptom onset/"last-known normal", prior to thrombolysis or interventional treatment. Patient data from the medical record were reviewed and recorded, including (1) National Institutes of Health Stroke Scale (NIHSS), (2) clinical laboratory analyses, including white blood cell differential, and (3) brain imaging (CT/MRI). Stroke-free control subjects were eligible for

recruitment if the following inclusion criteria were met: (1) age $\geq 18$ years, and (2) no 
history of AIS, transient ischemic attack, brain injury, or other overt central nervous system disease, and (3) recent hospitalization. Medical histories were obtained directly from stroke-free control subjects; however, complete access to medical records of control subjects was not available.

\section{Blood Sample Collection}

Peripheral venous whole blood was drawn from controls and from stroke subjects no later than 24 hours of stroke symptom onset. Approximately $8 \mathrm{~mL}$ of whole blood was collected into EDTA vacutainers, gently inverted 8-10 times and remained at room temperature (RT) for 20 minutes prior to processing.

\section{Fixation of Leukocytes from Whole Blood}

Whole blood samples were processed within one hour of collection. Whole blood was mixed with ACK lysis buffer (Life Technologies) to lyse red blood cells from each sample per manufacturer's instructions. Following RBC lysis, leukocytes were pelleted and fixed in preparation for surface and intracellular staining by Flow Cytometry. Leukocytes were resuspended in $2 \mathrm{~mL}$ PBS, $2 \mathrm{~mL}$ neutral-buffered formalin (NBF), vortexed immediately following addition of NBF, and incubated for 10 minutes at RT. Following incubation, leukocytes were pelleted and resuspended in cell culture preservation media, aliquoted, and placed in a cryopreservation container for $\sim 6$ hours before moving samples to $-80^{\circ} \mathrm{C}$ until analysis for fixed-rate freezing.

Determination of ARG1 by Flow Cytometry 
Fixed leukocytes were isolated using surface markers and intracellular ARG1 was stained and measured by Flow Cytometry. ARG1 was measured in T lymphocytes, monocytes, and neutrophils. The surface marker CD3 was used to stain T lymphocytes, CD14 for monocytes, and CD15 for neutrophils. Each leukocyte subpopulation was also stained for intracellular ARG1-APC. APC fluorescence was determined by Flow Cytometry and used to quantify ARG1 in each leukocyte population.

\section{Statistical Analysis}

Mann-Whitney $U$ Tests were used to compare intracellular ARG1 between AIS and control groups and also between mild and severe AIS groups. For group comparisons, mild and moderate AIS were combined to compare against severe AIS. Linear regression analysis was used to identify statstically significant relationships between groups when controlling for confounding regressors, specifically age, sex, hypertension, diabetes, prior stroke, and dyslipidemia.

\section{Results}

\section{Clinical Characteristics}

A total of 26 subjects were included in this study ( $n=12$ AIS, $n=14$ control). The demographic information for both AIS and control is included in Table 1. There were several significant differences between AIS and control subjects. Control subjects were significantly younger than AIS patients $(p=0.002)$ and AIS patients had a significantly larger proportion of patients with hypertension $(p=0.003)$ and patients who had suffered a previous ischemic stroke $(p=0.019)$ (Table 1$)$. In the AIS group, there were 3 mild 
severity, 3 moderate severity, and 6 severe severity cases. Further, the mean NIHSS of the AIS group was $9.5 \pm 5.8$ (mean \pm SD).

Relationship between Whole Blood ARG1 Expression and Neutrophil and Monocyte ARG1 Protein Levels

There is no relationship between whole blood ARG1 and neutrophil ARG1 in the total subject cohort $(r=0.254, p=0.295)$ (Figure 1C). However, when the total group is split into AIS and control subjects, there are relationships between whole blood ARG1 and neutrophil ARG1 in each group separately. Specifically, there is a weak relationship between whole blood ARG1 and neutrophil ARG1 in AIS patients $(r=0.642, p=0.119)$ (Figure 1A), and there is a weak relationship between whole blood ARG1 and neutrophil ARG1 in control subjects $(r=0.431, p=0.162)$ (Figure 1B). There was no relationship between whole blood ARG1 and monocyte ARG1 within any of the group comparisons (not shown). There was also no relationship between total leukocyte ARG1 protein (neutrophil + monocyte ARG1 protein) and whole blood ARG1 expression (Figure 2).

Neutrophil and Monocyte ARG1 in AIS and Control Subjects

No intracellular ARG1 was detected in the lymphocyte subpopulation. Intracellular ARG1 was detected in both the neutrophil and monocyte fractions in both AIS and control patients; however, neither neutrophil nor monocyte ARG1 levels significantly differed between AIS and control (Figure 3). Further, in both AIS and control, ARG1 was detected at a higher level in the neutrophil fraction compared to the monocyte fraction.

Neutrophil and Monocyte ARG1 in Mild and Severe AIS Patients 
Within the AIS group, neutrophil ARG1 was significantly higher in the severe group compared to the mild AIS group $(p=0.008)$ (Mean neutrophil ARG1 severe $=665 \pm 117$; mean neutrophil ARG1 mild/moderate $=453 \pm 108$ ) (Figure 4). Further, after linear regression analysis controlling for confounding variables, stroke severity remains a significant predictor of neutrophil ARG1 $(p=0.053)$.

\section{Discussion}

This is the first study to quantify intracellular ARG1 protein by Flow Cytometry in AIS patients. Previous reports have established that $A R G 1$ mRNA expression is highest in neutrophils and second highest in monocytes compared to other leukocyte subsets in healthy control subjects; however, these observations were not performed in AIS patients. The first aim of this study was to determine if ARG1 protein was present in neutrophils and monocytes, and if present, to establish whether ARG1 was higher in neutrophils compared to monocytes. We found that in both AIS and control subjects, ARG1 protein is expressed by both neutrophils and monocytes, and the neutrophil ARG1 protein expression is significantly higher than in monocytes. These findings confirm and also expand upon the previous observations that $A R G 1$ mRNA expression was highest in the neutrophils, and this relationship remains unchanged in response to AIS. Based on the previous reports of elevated ARG1 expression in the whole blood of AIS patients compared to control, we hypothesized that this elevated whole blood ARG1 expression preceded upregulation of neutrophil $A R G 1$ expression, and subsequently neutrophil ARG1 protein expression in AIS compared to controls. 
Contrary to our hypothesis, we observed no significant differences in neutrophil ARG1 protein expression between AIS and control subjects. While this was unexpected, based on previous gene expression data, there are several plausible explanations for this discrepant finding. First, the sample size in this study is extremely small, such that group comparisons should be made cautiously, as outliers can strongly influence datasets with a small number of subjects. To this end, it may be possible that the extreme values for neutrophil ARG1 in each group masked the true median values for neutrophil ARG1. Future studies with a larger sample size are warranted to further explore this hypothesis. Second, the control group utilized in this study consisted largely of subjects with metabolic syndrome (MetS). While MetS embodies AIS risk factors, making them a suitable control group, the AIS risk factors for stroke, such as hypertension and obesity, may contribute to altered neutrophil ARG1 expression. There have been no studies evaluating the effect of AIS risk factors on ARG1 or neutrophil ARG1 protein to support this hypothesis, and further studies would be required to determine the effect of AIS risk factors on ARG1 expression as a whole.

Also contrary to our hypothesis, there was no relationship between whole blood ARG1 expression and neutrophil ARG1 protein expression. Again, while in disagreement with our hypothesis, there are several possible explanations for this finding. First, because changes ARG1 mRNA expression precede changes in both systemic and neutrophil ARG1 protein expression, it may be possible that it is too early for a relationship between ARG1 mRNA expression and neutrophil ARG1 protein to be observed. For example, it may be possible that $A R G 1$ mRNA expression would be correlated with neutrophil ARG1 quantified at a future time point; however in this study both ARG1 mRNA and neutrophil 
ARG1 were quantified at the same time point. It is also possible that whole blood ARG1 mRNA expression is not correlated with neutrophil ARG1 because whole blood ARG1 mRNA expression contributes to systemic ARG1 protein upregulation in cells other than neutrophils. We attempted to address this by adding neutrophil and monocyte ARG1 together as a measure of "total" leukocyte ARG1. This is a loose definition of total leukocyte ARG1, as there may be low levels of ARG1 protein expression from other leukocyte subpopulations. Regardless, there was no relationship between our total leukocyte ARG1 protein and whole blood ARG1 mRNA expression. This suggests that ARG1 mRNA expression may contribute to translation/upregulation of ARG1 protein in cell types other than leukocytes, such as red blood cells. Future studies should expand the number of leukocyte subpopulations analyzed for ARG1 protein and also address upregulation of ARG1 in red blood cells.

Despite the previous findings, concordant with our hypothesis, we did observe a statistically significant difference in neutrophil ARG1 between mild and severe AIS. Specifically, ARG1 protein is higher in neutrophils in severe AIS compared to mild AIS. This finding suggests there may be an upregulation in neutrophil ARG1 in response to stroke that is dependent on AIS severity. We have previously shown that whole blood ARG1 mRNA expression is positively correlated with stroke severity, and taken together with this novel finding of increased ARG1 protein in neutrophils supports the concept that increased ARG1 protein contributes to increased AIS severity. This finding should be interpreted cautiously, given the previous finding that there was no relationship between whole blood ARG1 expression and neutrophil ARG1 protein expression. 
Overall, the results of this study suggest that ARG1 protein expression is highest in neutrophils compared to other leukocyte populations. Neutrophil ARG1 may also increase in response to brain damage in severe AIS. Because increased ARG1 protein released from neutrophils has been shown to exacerbate AIS damage in a rodent model of stroke, these findings suggest that inhibition of systemic ARG1 protein may improve AIS outcome, and neutrophils may be a therapeutic target for ARG1 inhibition. 


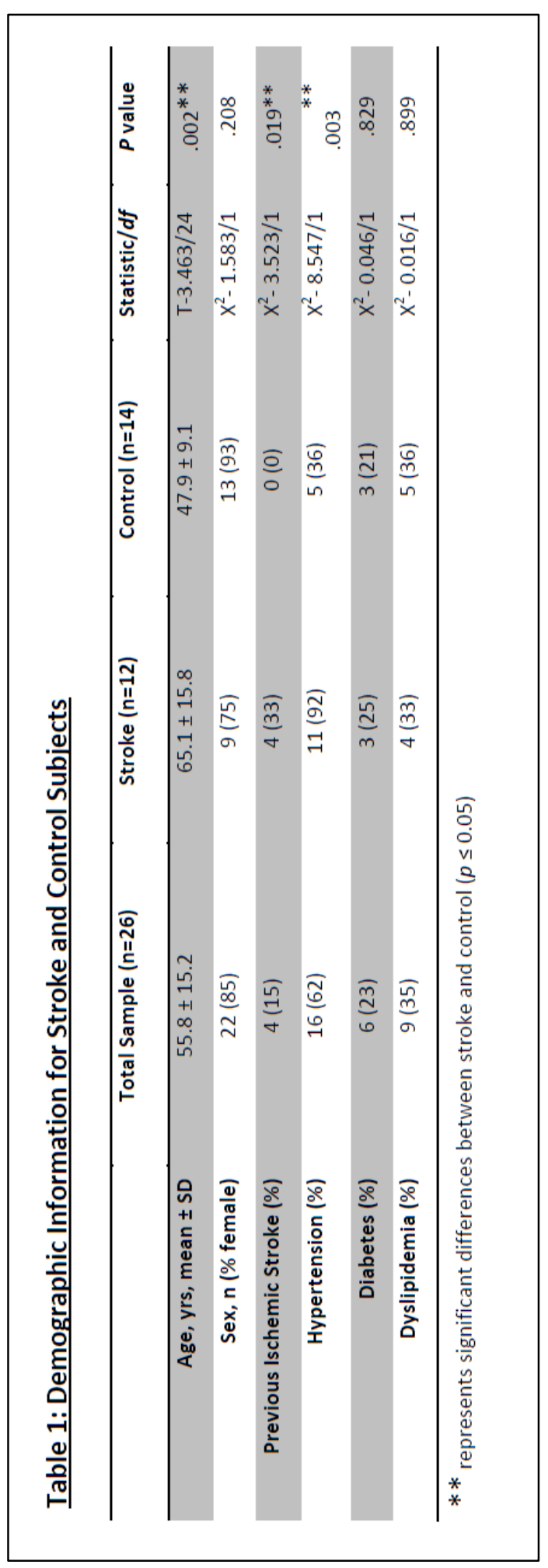



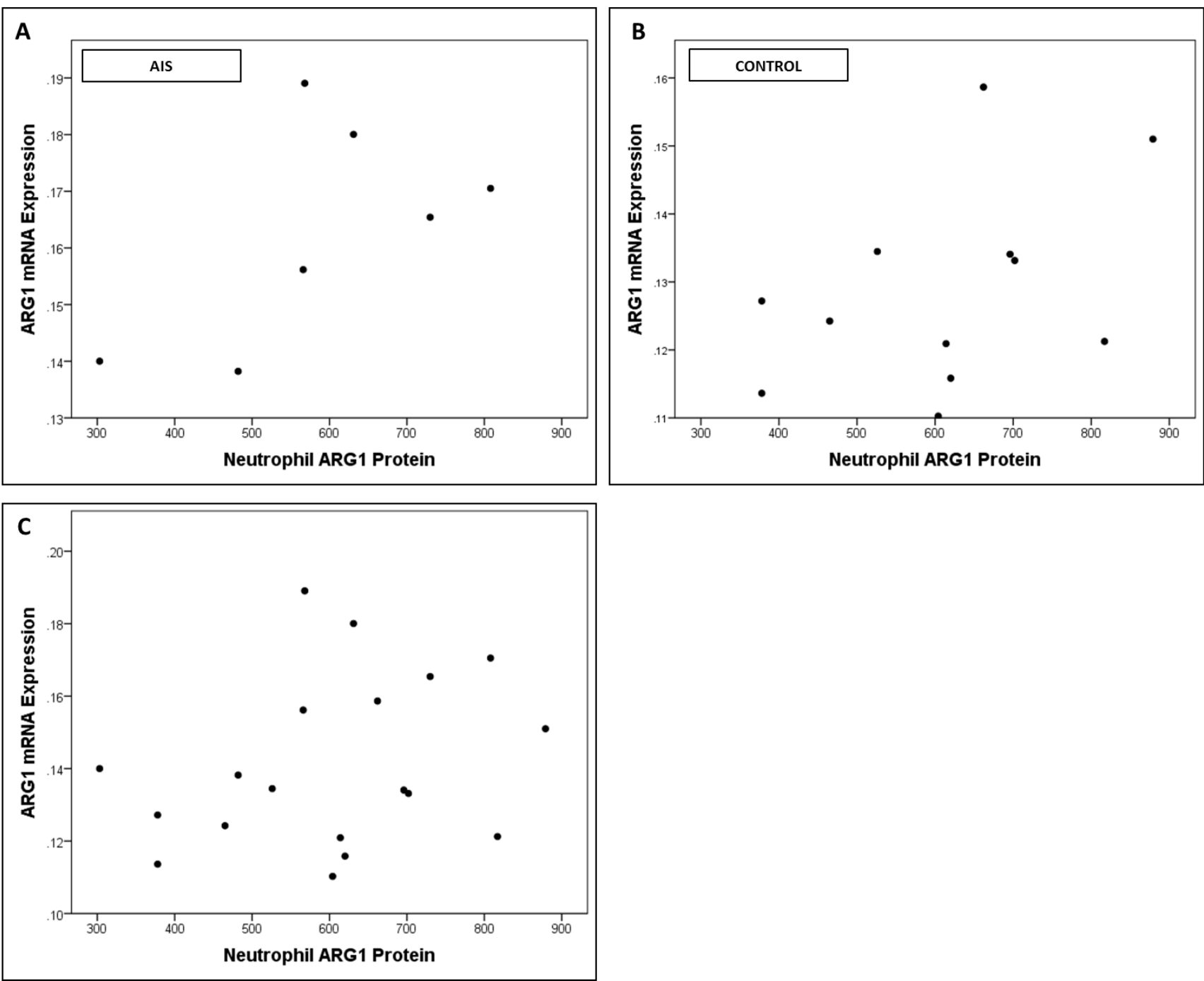

Figure 1: Relationship Between Whole Blood ARG1 mRNA Expression and Neutrophil ARG1 Protein in AIS and Control.

(A) There is a weak relationship between whole blood ARG1 and neutrophil ARG1 in AIS patients $(r=0.642, p=0.119)$.

(B) There is a weak relationship between whole blood ARG1 and neutrophil ARG1 in control subjects $(r=0.431, p=0.162)$.

(C) There is no relationship between whole blood $A R G 1$ and neutrophil ARG1 in the total subject cohort (control and AIS combined) $(r=0.254, p=0.295)$. 


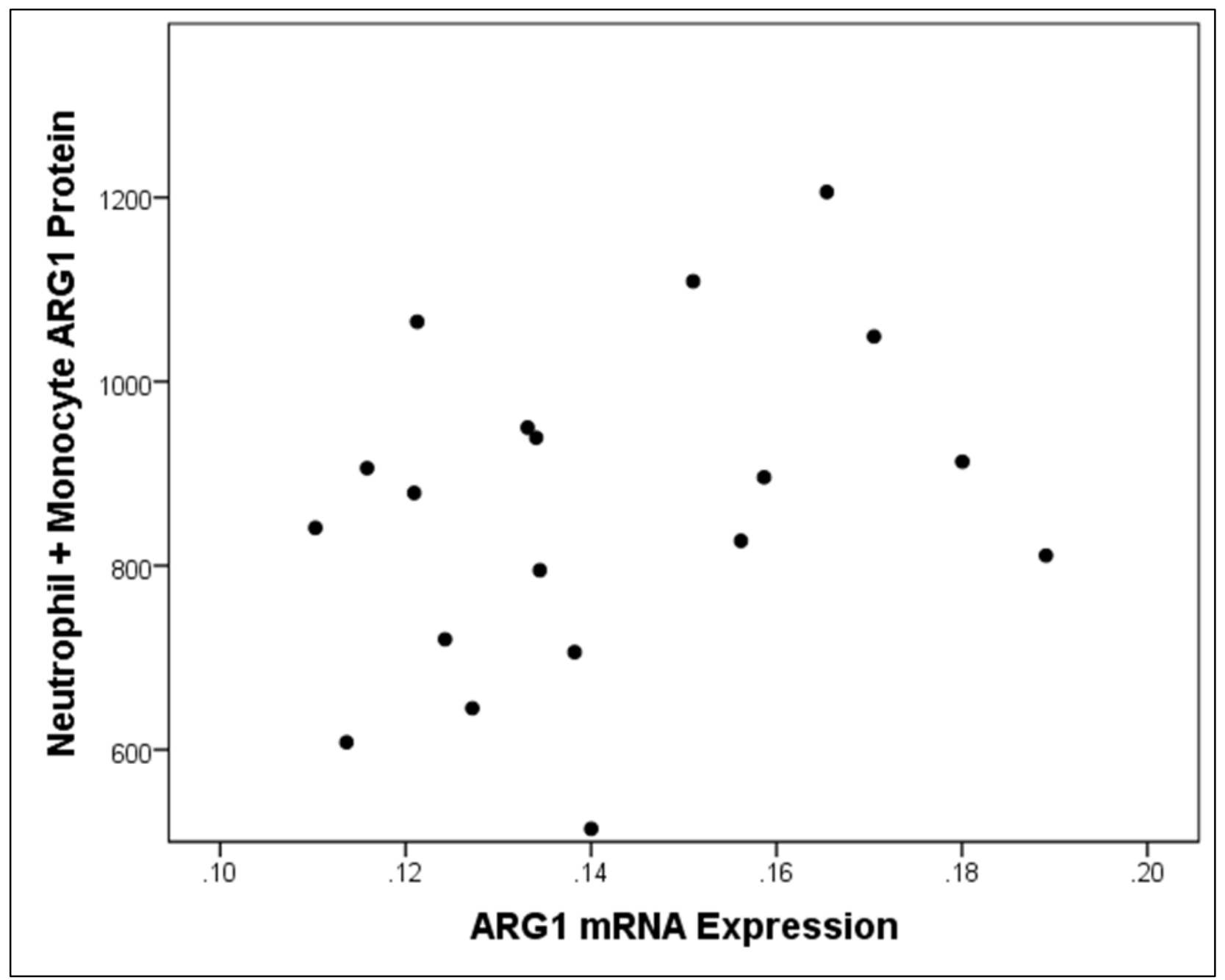

Figure 2: Relationship between Total Leukocyte ARG1 Protein and Whole Blood ARG1 mRNA Expression. There is a weak relationship between total leukocyte ARG1 protein (neutrophil + monocyte ARG1 protein) and whole blood ARG1 mRNA expression $(r=0.319, p=0.184)$. 


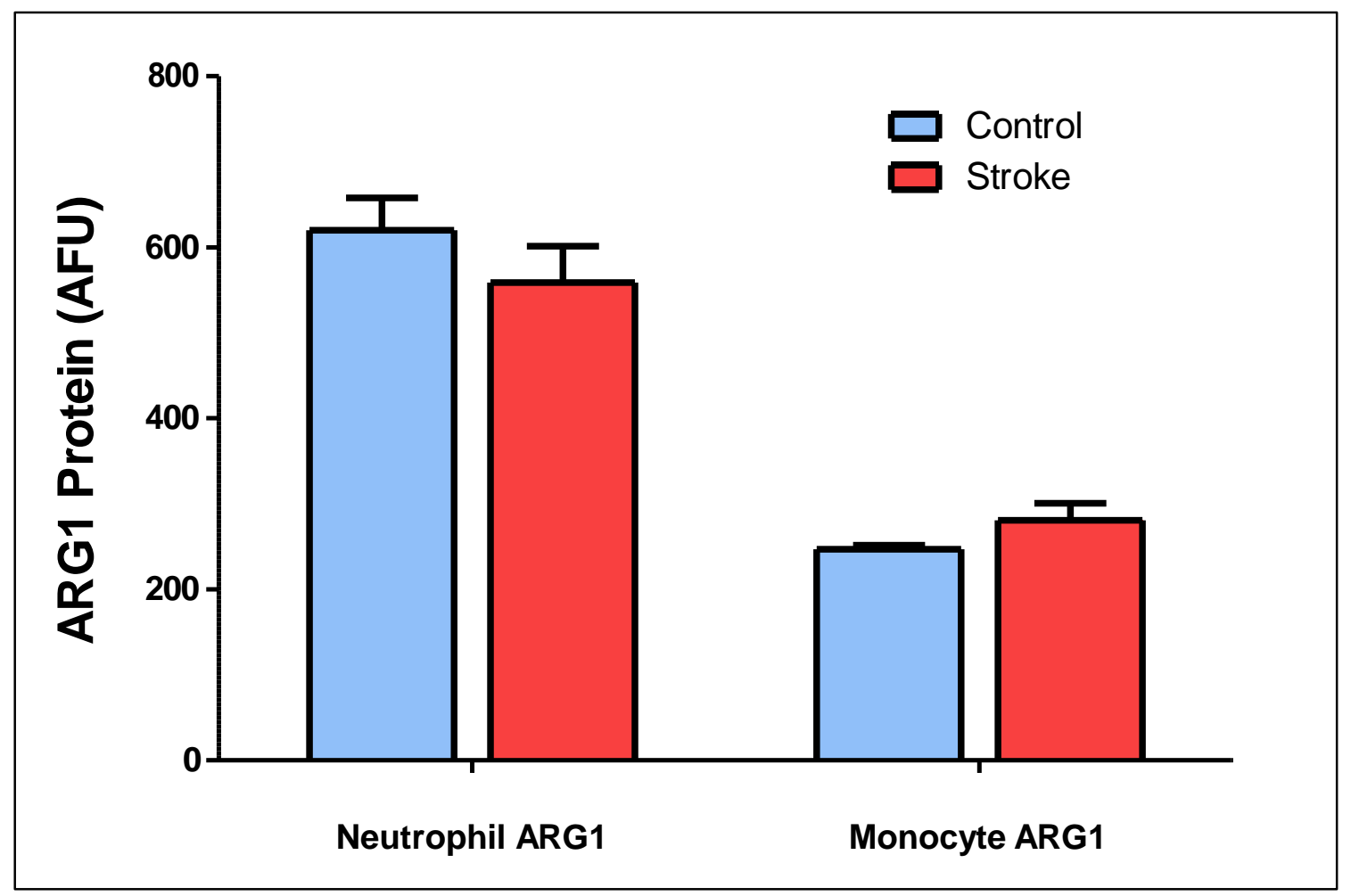

Figure 3: Intracellular ARG1 Staining in Leukocyte Subpopulations in AIS and Control Subjects. Intracellular ARG1 was detected in both the neutrophil and monocyte fractions in both AIS and control patients; however, neither neutrophil nor monocyte ARG1 levels significantly differed between AIS and control (mean neutrophil ARG1 AIS=558 \pm 154 ; mean neutrophil ARG1 control=620 \pm 145 ; mean monocyte ARG1 AIS=281 \pm 72 ; mean monocyte ARG1 control=248 \pm 18 ). 


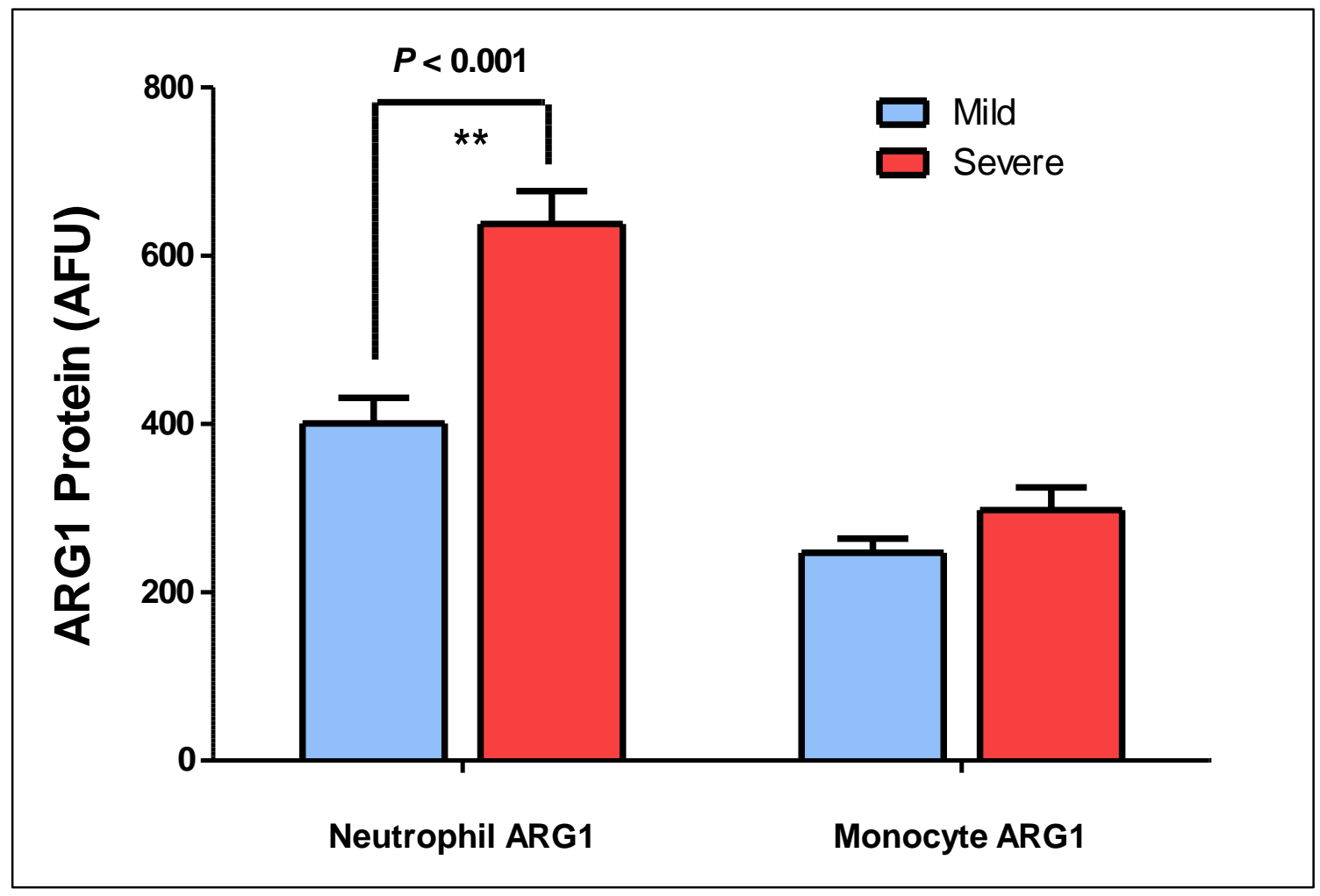

Figure 4: Intracellular ARG1 Staining in Leukocyte Subpopulations in AIS Patients by Stroke Severity. Within the AIS group, neutrophil ARG1 was significantly higher in the severe group compared to the mild AIS group $(p=0.008)$ (Mean neutrophil ARG1 severe $=665 \pm 117$; mean neutrophil $A R G 1$ mild $/$ moderate $=453 \pm 108$ ). 


\section{References}

1. Kamel $\mathrm{H}$, ladecola $\mathrm{C}$. Brain-immune interactions and ischemic stroke: Clinical implications. Arch Neurol. 2012;69:576-581

2. Vogelgesang A, Dressel A. Immunological consequences of ischemic stroke: Immunosuppression and autoimmunity. J Neuroimmunol. 2011;231:105-110

3. Wang $Q$, Tang $X N$, Yenari MA. The inflammatory response in stroke. J Neuroimmunol. 2007;184:53-68

4. Offner $\mathrm{H}$, Vandenbark AA, Hurn PD. Effect of experimental stroke on peripheral immunity: Cns ischemia induces profound immunosuppression. Neuroscience. 2009;158:1098-1111

5. Petrone AB, O'Connell GC, Regier MD, Chantler PD, Simpkins JW, Barr TL. The role of arginase 1 in post-stroke immunosuppression and ischemic stroke severity. Transl Stroke Res. 2015

6. Barr TL, Conley Y, Ding J, Dillman A, Warach S, Singleton A, et al. Genomic biomarkers and cellular pathways of ischemic stroke by rna gene expression profiling. Neurology. 2010;75:1009-1014

7. Tang $Y, X u H, D u X$, Lit L, Walker W, Lu A, et al. Gene expression in blood changes rapidly in neutrophils and monocytes after ischemic stroke in humans: A microarray study. J Cereb Blood Flow Metab. 2006;26:1089-1102

8. Munder M, Schneider H, Luckner C, Giese T, Langhans CD, Fuentes JM, et al. Suppression of t-cell functions by human granulocyte arginase. Blood. 2006;108:1627-1634 


\section{CHAPTER 7: GENERAL DISCUSSION}

The specific aims of this dissertation were to: (1) Determine the Relationship Between ARG1, NLR, and AIS severity, (2) Characterize the Role of ARG1 in Mediating CV Function following AIS, and (3) Determine the Cellular Origin of ARG1 and Functional Role of ARG1 in AIS. Chapters 2 and 3 represent data to support specific aim 1, chapters 4 and 5 represent data to support specific aim 2, and chapter 6 supports specific aim 3.

\section{Chapter Summaries}

\section{Chapter 2}

The results of chapter 2 were published in the peer-reviewed journal, Translational Stroke Research. The purpose of this study was: (1) To examine the relationship between ARG1, NLR, and AIS severity. We hypothesized that increased ARG1 expression and serum protein activity is associated with an increased NLR, giving rise to increased AIS severity and poor outcome; (2) To validate the role of ARG1 as a novel biomarker of immune suppression in AIS; and (3) To Utilize Principal Component Analysis (PCA) to statistically model multiple gene expression changes following AIS.

In support of our hypothesis, we found that both increased ARG1 mRNA and serum activity are positively correlated with AIS severity and NLR, and increased NLR was associated with increased AIS severity. PCA also supported a model of increased ARG1 and MMP9 and decreased CCR7 as a pattern of expression associated with AIS severity and NLR. 
This study was characterization of the relationship between ARG1, NLR, and AIS severity; however, while the associations were strongly significant, there is no evidence for a functional role of the biomarkers described in this study. This is a limitation of biomarker studies as a whole, which is especially true for gene expression analysis. Because changes in gene expression are not always strongly correlated in changes in protein expression or activity, functional interpretation of gene biomarker analysis should be approached cautiously. With that being said, in addition to gene biomarkers, we also measured serum arg1 activity to provide more insight into the functional role of ARG1. Serum ARG1 activity was strongly correlated with ARG1 mRNA expression that suggests for ARG1, changes in gene expression are correlated with changes in protein activity. Further, ARG1, MMP9, and s100a12 were found to be the most significantly elevated genes in our panel in AIS compared to control. All 3 of these genes are highly expressed by neutrophils, so the possibility exists that increases in these biomarkers reflect an increase in neutrophil count, rather than a functional mechanism contributing to AIS severity. It is my opinion that changes in ARG1 and MMP9 represent functional changes in AIS, whereas s100a12 may be simply a marker of neutrophil count following AIS. S100a12 is a calcium-binding protein that is constitutively expressed by neutrophils and has not been shown to have any relationship to AIS in any other context. MMP9 is also constitutively expressed by neutrophils; however, MMP9 is highly expressed by several leukocytes, especially innate immune cells. MMP9 release aids in cell migration into the brain following AIS, but also contributes to BBB disruption and AIS severity. Addition of serum MMP9 activity would be useful to determine if MMP9 has a potential functional role 
in AIS; however, the functional role of MMP9 in AIS has been extensively described, so it is unlikely that MMP9 is only a marker of neutrophil count.

Another limitation of this study is that the AIS cases were not split by AIS subtype, and there have been several studies that suggest that different patterns of biomarker expression are associated with different AIS subtypes. It may be possible that in this small sample size, the gene expression patterns we observed were associated with a specific AIS subtype, rather than AIS as a whole, if this dataset contains an unequal distribution of AIS subtypes. For example, large artery AIS is associated with carotid atherosclerosis, and because we know that ARG1 expression contributes to atherosclerosis formation, it may be possible that differences in ARG1 expression are due to risk factors prior to stroke, rather than an acute response to AIS itself. Unfortunately, Ruby Memorial Hospital does not classify AIS subtype using TOAST or any other sub-typing system; therefore, we were unable to consider this in our analysis. Further, given the limited sample size, it would have been difficult to perform meaningful statistical analysis on small groups split by AIS subtype.

Lastly, we were unable to obtain NLR and serum ARG1 activity in our control group. This was a logistical issue related to how our control patients were collected. A bulk of the control subjects included in this analysis were obtained via recruitment for the study described in Chapter 5, and because we were recruiting a remote, rural setting, serum isolation was not feasible for these samples. Further, while NLR may have been obtained, the NLR was not a primary outcome measurement during the planning of this study, but will likely be added to the protocol for future studies. This would be useful for several reasons, but it would be crucial to measure neutrophil count in controls, and my 
hypothesis is that neutrophil counts would be similar between groups, however that ARG1 would still remain elevated in the AIS group, indicating a functional role of ARG1, independent of neutrophil count alone.

\section{Chapter 3}

The aim of this study was to expand upon the finding that baseline NLR is associated with AIS severity and outcome and determine if measuring the NLR after thrombolysis or at several time points following AIS provide greater insight into stroke severity and outcome than baseline NLR. We hypothesized that an increase in NLR following thrombolysis is associated with increased severity and poor outcome, regardless of the baseline NLR value prior to treatment.

While the findings of this study lacked statistical significance, there is evocative evidence to suggest that not only can the baseline NLR be used to stratify AIS patients according to severity, but also that measuring the change in NLR following thrombolysis may also be indicative of acute severity, and may be a stronger indicator of outcome compared to baseline NLR.

\section{Chapter 4}

The aims of this study were to: (1) Characterize the changes in ARG1 expression that occur in response to AIS, (2) Characterize the changes in cfPWV that occur in response to AIS, and (3) To determine if ARG1 expression may be a functional mediator of vascular function, specifically cIMT and cfPWV, following AIS.

We hypothesized that increases in both ARG1 expression and cfPWV would be associated with increased baseline stroke severity and poor outcomes. We also 
hypothesized that ARG1 expression is correlated with cfPWV at both baseline and during AIS recovery.

Our results supported our hypotheses, specifically, there is a strong unadjusted relationship between baseline ARG1 expression and baseline NIHSS. In mild AIS, cfPWV remains stable from baseline to 30 days post-stroke. In contrast, cfPWV increased from baseline to 30 days post-stroke in severe AIS. Lastly, there appears to be a relationship between $\triangle A R G 1$ and $\triangle$ cfPWV from baseline to 30 days post-stroke. However due to the small sample size further study in this area is warranted to back up our initial findings.

\section{Chapter 5}

The goal of this study was to characterize the changes in ARG1 expression, as well as cIMT and cfPWV, following a 10-week lifestyle modification. We hypothesized that decreases in CV risk factors, such as cIMT, cfPWV, blood pressure, and body weight, will correlate with decreases in ARG1 expression.

First, similar to previous reports, we confirmed the strong relationship between CIMT and cfPWV. In contrast to our hypothesis, there were no associations between decreased body weight, cholesterol, triglycerides, increased functional exercise capacity, and cfPWV. However, in support of our primary hypothesis, we did observe a strong correlation between the 10-week $\triangle \mathrm{cfPWV}$ and $\triangle A R G 1$ expression, supporting our hypothesis that ARG1 may be a functional mediator of cfPWV.

\section{Chapter 6}

The aims of this study were to expand upon previous studies to (1) Determine whether ARG1 protein, in addition to mRNA expression, is highest within neutrophils compared to other leukocyte fractions, (2) Determine if neutrophil ARG1 protein levels 
differ between AIS and control and (3) Characterize the change in neutrophil ARG1 protein that may occur with increasing AIS severity. We hypothesized that ARG1 protein would be highest in neutrophils compared to other leukocyte populations in both AIS and control; however, ARG1 protein levels would be higher in the neutrophils of AIS patients compared to controls. Further, we hypothesized that within the AIS group, neutrophil ARG1 protein levels will be higher in severe AIS compared to mild.

We found that in both AIS and control subjects, ARG1 protein is expressed by both neutrophils and monocytes, and the neutrophil ARG1 protein expression is significantly higher than in monocytes. Contrary to our hypothesis, we observed no significant differences in neutrophil ARG1 protein expression between AIS and control subjects. Also contrary to our hypothesis, there was no relationship between whole blood ARG1 expression and neutrophil ARG1 protein expression.

\section{Limitations}

Small Sample Size

An overall limitation of this dissertation was small sample sizes, as compared to other clinical studies. The following sections, including power analyses, barriers to subject recruitment, and future study design will specifically address sample size concerns, provide an explanation for sample sizes, and also provide a strategy to improve upon these limitations in future studies.

Power Analyses 
Post-hoc power analyses were conducted to determine achieved power, given alpha level, sample size, and effect size using $\mathrm{G}^{\star}$ Power3.

\section{Chapter 2}

We performed two independent power analyses for the two primary aims of the study: (1) To evaluate the relationship of ARG1 and stroke severity, as measured by NIHSS, and (2) To compare ARG1 expression between stroke and control. Using a linear regression model, controlling for seven predictors (age, sex, smoking, hypertension, hyperlipidemia, diabetes and prior stroke), we determined that our sample size of $n=26$ and effect size 1.5 was sufficient to detect differences in ARG1 expression along the $\mathrm{NIH}$ stroke severity score scale with $88 \%$ power at an alpha level of 0.05 . Using a linear regression model, controlling for eight predictors (case (control v. stroke), age, sex, smoking, hypertension, hyperlipidemia, diabetes, and prior stroke), we determined that our sample size $n=45$ and effect size 0.5 was sufficient to detect differences in ARG1 expression between stroke and controls with $99 \%$ power at an alpha level of 0.05 . Of all the chapters in this dissertation, this was the only study that achieved desired statistical power.

\section{Chapter 3}

We determined that our sample size of $n=18$ and effect size of $f=0.25$ was able to detect differences in NLR between mild, moderate, and severe AIS groups with only $12 \%$ power at an alpha level of 0.05 , using a repeated measures ANOVA. A sample size of 159 subjects would be necessary to a small effect $(\mathfrak{f}=0.25)$ between groups with $80 \%$ power at an alpha level of 0.05 .

\section{Chapter 4}


We determined that our sample size of $n=12$ and effect size of $f=0.3$ was able to detect differences in ARG1 expression in relationship to cIMT and cfPWV with only $817 \%$ power at an alpha level of 0.05 . A sample size of 82 subjects would be necessary to a small effect $(\mathrm{f}=0.3)$ with $80 \%$ power at an alpha level of 0.05 .

\section{Chapter 5}

We determined that our sample size of $n=12$ and effect size of $f=0.3$ was able to detect differences in ARG1 expression in relationship to cIMT and cfPWV with only $817 \%$ power at an alpha level of 0.05 . A sample size of 82 subjects would be necessary to a small effect $(\mathrm{f}=0.3)$ with $80 \%$ power at an alpha level of 0.05 .

\section{Chapter 6}

We determined that our sample size of $n=26$ and effect size $f=0.25$ was able to detect differences in neutrophil ARG1 levels between AIS and control with only $23 \%$ power at an alpha level of 0.05 , using a repeated measures ANOVA. We also determined that our sample size of $n=12$ AIS patients and effect size $f=0.25$ was able to detect differences in neutrophil ARG1 levels between mild and severe AIS with only $12 \%$ power at an alpha level of 0.05 , using a repeated measures ANOVA. A sample size of 128 subjects would be necessary to a small effect $(\mathfrak{f}=0.25)$ between both stroke and control and mild and severe AIS, with $80 \%$ power at an alpha level of 0.05 .

Barriers to Subject Recruitment

Because Ruby Memorial Hospital receives 1200 stroke pages annually and it is estimated that roughly one third or 400 of those pages are stroke cases. Of that estimated 400 cases, approximately 320 are ischemic strokes. With that being said, our laboratory is often asked why our annual subject enrollment is substantially less than the number of 
ischemic stroke patients presenting to Ruby Memorial Hospital. There are several factors that can be considered as barriers to subject recruitment in our studies.

The first and largest barrier to subject recruitment is the requirement for an imaging-confirmed stroke diagnosis, in order to be eligible for enrollment in our studies. This means that no stroke patient is eligible for enrollment into our study without a CT or MRI image, confirming the presence of an ischemic infarct, and this image must also be obtained prior to intervention, such as tPA administration or mechanical clot removal. While stroke imaging is certainly a part of clinical care at Ruby Memorial Hospital, not all patients that will be discharged with a stroke diagnosis, and therefore contribute to the annual number of stroke cases reported at Ruby, are confirmed by imaging. There are a substantial number of cases that are diagnosed as stroke, on the basis of clinical presentation and physician impression, even in the absence of positive imaging.

The second largest barrier to recruitment is the time at which stroke patients present to the emergency department. Our laboratory staff and work coordinators typically work a 9am-5pm schedule, thus stroke patients who arrive between the hours of $5 \mathrm{pm}-9$ am the next morning are difficult to enroll in the study. Because we are approved to enroll stroke patients within 24 hours of symptom onset, some of these patients who arrive "after-hours" may still be eligible for our studies if they have not undergone any intervention, such as tPA administration or mechanical clot removal; however, a large number of patients will have already treated in the time that our staff is unavailable for recruitment. To increase subject enrollment during our typical working hours, for a short period of time during the duration of this dissertation, we had a nurse coordinator who was in charge of recruitment from 7pm-8am, and we expected to see dramatic increases 
in subject recruitment. To our surprise, this extra staff did not lead to a large increase in subject recruitment, thus while it may be a strategy to employ in the future, the largest barrier to subject recruitment was not a matter of laboratory staff availability and effort, but due to the first barrier to recruitment mentioned above.

\section{Future Directions}

\section{Study Design}

In addition to simply increasing sample size in our studies, future studies should also use different clinical trial designs to make even small sample sizes more meaningful. An adaptive clinical trial is a clinical trial that is guided by prospectively planned interim data analyses during the course of the trial that may prompt changes in the trial design to make the studies more efficient. These changes in trial design can include, but are not limited to, changes in eligibility criteria, treatment regimens, sample size, and statistical interpretation. For example, an adaptive clinical trial monitoring the efficacy of a new therapeutic agent for stroke may be approved at the start of the trial for multiple doses, but if an interim analysis conducted into the study suggests that only certain doses are effective, the trial will then be modified, such that patients enrolled after the interim analysis will receive the doses deemed to be effective in the interim analysis. These frequent interim analyses and adaptations make clinical trials more efficient by both

reducing the number of patients needed to show efficacy in a clinical trial and by reducing the duration of the trial. This improved efficacy will result in a shorter amount of time that it takes for novel, beneficial therapeutic agents to reach the clinical population.

\section{Experimental Studies}




\section{CCR7}

CCR7 is a membrane-bound receptor located on T lymphocytes that is required for homing to secondary lymphoid organs prior to activation. We have shown that CCR7 expression is decreased in AIS compared to control, and CCR7 expression is negatively correlated with AIS severity. We have also established that CCR7 downregulation may be linked to an increase in ARG1 expression. Interestingly, while ARG1 has been shown to inhibit T cell proliferation by downregulation of the TCR complex, decreased CCR7 has not been implicated in immunosuppression in animal models of AIS. Further, there have been no studies evaluating CCR7 overexpression on AIS outcomes. It is unclear why CCR7 has been identified, as a biomarker in human AIS studies, yet has not surfaced in animal models of AIS, considering CCR7 is expressed and has the same function in both humans and animals. With that being said, many clinical trials for AIS therapeutics fail because animal models fail to translate to humans, whereas CCR7 may be a therapeutic target in AIS that is identifiable in humans, but lacks relevance in animals. My hypothesis is that because CD3 has not been shown to be significantly decreased in human AIS studies, ARG1 may induce immunosuppression may induce immunosuppression through downregulation of $\mathrm{CCR} 7$ rather than $\mathrm{CD} 3$ in humans.

\section{ARG1 Inhibition}

Given that ARG1 inhibition is currently in clinical trials for use in myocardial ischemiareperfusion injury, it is surprising that there have been no experimental models evaluating ARG1 inhibition in the context of AIS. Given the recent studies, evaluating the role of ARG1 in post-stroke immunosuppression, I believe that these studies may have already been performed and will be published in the near future. Given its success in myocardial 
ischemia-reperfusion injury and the data presented in this dissertation, I believe that ARG1 inhibition may be a therapeutic target, both acutely and chronically, following AIS.

In terms of chronic ARG inhibition, there have been no studies to evaluate what effect chronic ARG inhibition would have systemically. Theoretically speaking, ARG1 inhibition would result in increased bioavailability of $\mathrm{NO}$ and increased vasodilation. There are no instances where increased vasodilation could be harmful, so in my opinion, chronic ARG1 inhibition would have no ill effects on vascular function. There is a possibility that chronic ARG1 inhibition would lead to the development of autoimmune conditions, given the potential role of ARG1 as immunosuppressive. If ARG1 inhibition would be considered as a chronic treatment for CV risk factors or long-term following AIS, clinical trials would be needed to evaluate the safety and off target effects.

\section{Retrospective Analysis on AIS in Arginase Deficiency}

Along the lines of chronic ARG inhibition, while rare, there are reported cases of ARG deficiency in humans. This is a non-lethal, genetic disorder that generally requires no treatment, other than diet modification to limit protein, I.e. L-arginine, intake. While there is no screening data currently for this disorder, a retrospective analysis with data from these patients would be interesting to evaluate AIS incidence, severity, and recovery. It also supports the hypothesis that chronic ARG1 inhibition should have no severe side effects on the system.

\section{ARG1 Expression by Red Blood Cells}


While gene expression biomarkers in peripheral whole blood are considered to be reflective of changes in leukocytes, red blood cells have also been to express several mRNA molecules, despite the lack of a nucleus. In terms of AIS, the upregulation following AIS may be due to upregulation by red blood cells. This may explain why neutrophilinhibition therapies have been unsuccessful in AIS clinical trials may be partially due to the fact that ARG1 is still being generated by other sources, including RBCs. Isolation of RBCs in AIS patients and measuring ARG1 expression in RBCs following AIS in addition to WBC subtypes would be an easily performed experiment to address the role of RBCderived ARG1 in AIS.

\section{ARG1 Gene Expression on WBC Subsets following Stroke}

To our knowledge, there has been only one study evaluating ARG1 mRNA expression on individual leukocytes. This study only addressed leukocyte-ARG1 expression in 3 control subjects and determined that ARG1 is highest in neutrophils and monocytes; however, it is currently unknown whether ARG1 is upregulated in these cell types in a severitydependent manner following AIS or if ARG1 expression increased in other leukocytes following AIS.

\section{Preventing ARG1 Release for ARG1 Protein Analysis by Flow Cytometry}

This is logistic improvement from our studies performed in chapter 6 , determining the cellular source of ARG1 protein following AIS. This study addressed the hypothesis of a severity-dependent increase in ARG1 by neutrophils following stroke. While we did see an elevation in neutrophil ARG1 in severe compared to mild stroke, we did not see an increase in total stroke compared to control, which makes us interpret the severitydependent increase in ARG1 cautiously. When comparing AIS to control, we actually 
observed a non-significant decrease in neutrophil ARG1 in AIS compared to control. Because ARG1 is likely secreted from neutrophils in granules, it may be possible that we observed this expression because more ARG1 is released from neutrophils in AIS compared to control. To address this, isolated WBCs can be treated with a chemical that prevents proteins from being exported from the cell, thus secreted proteins can be quantified, because they accumulate in the cell. It is necessary to consider this in future studies.

\section{NLR at Multiple Time Points}

While we examined the NLR at both baseline and 48 hours following AIS, measuring the NLR at later time points, 7,30, and 90 days following AIS, may be more indicative of poststroke immunosuppression, as increased rate of infection in AIS patients is not observed until roughly one week following AIS. We have approval to collect NLR at these time points; however, because a WBC differential is not collected at these follow-up visits as a standard of clinical care, incomplete data on our patients made this analysis less meaningful.

\section{T Lymphocyte Subset Specific NLR}

In addition to the basic NLR described in this document, there are several variations of the NLR that may have more utility in AIS. The NLR referred to in this document is (total neutrophil count/ total lymphocyte count), thus will be influenced by both $B$ and $T$ lymphocyte counts. B lymphocytes have been shown to play a role in AIS, albeit less prominent than T lymphocytes, such that this version of the NLR may be complicated by the interplay between B and T lymphocytes. The first variation of the NLR that could be useful in stroke would be a T lymphocyte-specific NLR calculated by (total neutrophil 
count/ total T-lymphocyte count). Because decreased T lymphocyte counts and function are indicative of NLR, this variation of the NLR may be more predictive of post-stroke immunosuppression. Unfortunately, clinical WBC differentials do not distinguish B and T lymphocytes, thus determination of this NLR would likely be performed manually by flow cytometry. In my opinion, this variation of the NLR would have the most utility in predicting AIS severity and post-stroke immunosuppression, and it would be measured by (neutrophil count/T lymphocyte subset count). Because we know that different $T$ subsets play very different roles in AIS, these NLRs could yield very different information, especially if measured at multiple time points. I hypothesize that increased (neutrophil count/Treg count) would be most predictive of post-stroke immunosuppression and poor outcome, due to the beneficial role of TReg cells compared to other T subsets following AIS.

\section{The Effect of ARG1 on T Lymphocyte Subsets}

Similar to the direction suggested above, it is possible that ARG1 impacts T cell proliferation in a subset-dependent manner. For example, it may be possible that $T_{\text {reg }}$ cells are more susceptible to ARG1-mediated inhibition than CD8+ T cells or vice versa. Thus, it would also be important to examine the relationship of ARG1 to a T-subset specific NLR to provide better insight into the functional role of ARG1 in mediating the NLR. 


\title{
Ashley Brooke Petrone
}

\author{
1 Kensington St, Uniontown PA 15401 \\ Email: abpetrone@mix.wvu.edu \\ Phone: (304) 435-5507
}

\section{EDUCATION:}

Aug.2012 - Nov.2015

May 2012
$\mathrm{PhD}$, Neuroscience

West Virginia University: Morgantown, WV 26505

Bachelor of Science, Biology (Pre-medicine)

Gannon University: Erie, Pennsylvania

\section{TEACHING EXPERIENCE:}

Fall 2015

Course Coordinator/Instructor in PSYCH 301: Biological

Foundations of Behavior

West Virginia University, Department of Psychology

Fall 2014, Fall $2013 \quad$ Course Coordinator/Instructor in BMS 706: Cellular Methods

West Virginia University, Biomedical Science PhD Curriculum

Fall 2014

Teaching Assistant in Immunology Colloquium I

West Virginia University, Department of Immunology and Microbiology

Spring 2014, Spring 2015 Teaching Assistant in Microbiology Colloquium I

West Virginia University, Department of Immunology and Microbiology

\section{RESEARCH EXPERIENCE:}

\section{Dissertation Title: The Role of Arginase I in Ischemic Stroke and Cardiovascular Disease}

Dissertation Mentor: Dr. Taura L. Barr RN, PhD, WVU School of Nursing, Morgantown WV

Project Summary: My dissertation research focused on the role of an inflammatory protein, Arginase 1, on Ischemic Stroke Risk, Severity, and Recovery. We hypothesized that increased Arginase 1 would result in immune dysfunction that is associated with increased acute stroke severity and poor recovery. To complete this project, I collected blood samples and performed cardiovascular assessments on ischemic stroke patients upon admission to the hospital and at several time points during their hospital stay and return visits out to 90 days post-stroke. Arginase 1 was then quantified in the blood and correlated with ischemic stroke severity and outcome measures, vascular function, and demographic information. My dissertation research culminated in a first author publication in the peer-reviewed journal, Translational Stroke Research. 


\section{LABORATORY SKILLS:}

- Sterile cell culture, Western Blot, PCR, Real-time PCR, Phlebotomy, Carotid Ultrasound, Pulse Wave Velocity and Analysis, Immunohistochemistry, Fluorescence Microscopy, Flow Cytometry, Statistical Analysis using SPSS and G*Power

\section{RESEARCH PUBLICATIONS:}

Petrone AB, O'Connell GC, Regier M, Chantler PD, Simpkins JW, and Barr TL (2015). The Role of Arginase 1 in Post-Stroke Immunosuppression and Ischemic Stroke Severity. Translational Stroke Research.

Petitte T, Mallow J, Barnes E, Petrone AB, Barr TL, and Theeke L. (2015). A Systematic Review of Loneliness and Common Chronic Physical Conditions in Adults. The Open Psychology.

Petrone AB, Rudy CC, Barr TL, Simpkins JW, and Reed MN (2014). Neuroprotective Effects of Estrogen Following Neural Injury. Estrogen Effect on Traumatic Brain Injury. Vassar College.

Petrone AB, Gatson JW, Simpkins JW, and Reed, MN. (2014). Non-Feminizing Estrogens: A Novel Neuroprotective Therapy. Molecular and Cellular Endocrinology .

Petrone AB, Simpkins JW, and Barr TL. (2014). Estrogens and Inflammation: Implications for Ischemic Stroke. Journal of Aging and Diseases.

Barr TL, VanGilder RL, Jia Z, Sieberg R, Petrone AB, Chantler PD, and Huang C (2014). Systemic Transcriptional Alterations of Innate and Adaptive Immune Signaling Pathways in Atherosclerosis, Ischemic Stroke, and Myocardial Infarction. Journal of Bioanalysis and Biomedicine.

\section{INVITED PRESENTATIONS:}

Petrone AB, O'Connell GC, and Barr TL. 2015. Arginase 1 Expression Mediates Stroke Severity and T Cell Proliferation in Ischemic Stroke. E.J. Van Liere Research Convocation. West Virginia University.

Petrone AB, VanGilder R, Simpkins JW, and Barr TL. 2014. Sex Differences in IL-6 Levels Following Stroke: Implications for Stroke Severity and Outcome. Council for the Advancement of Nursing Science Annual Conference, Washington D.C.

\section{AWARDS/HONORS:}

March 2015

March 2015

Feb. 2015
Honorable mention - WVU 3-Minute Thesis Competition. West Virginia University. Behavioral and Biomedical Science Training Scholarship. West Virginia University. $1^{\text {st }}$ place- Clinical/Translational Science Oral Presentation. Edward J. Van Liere Research Convocation, West Virginia University. 


\section{OTHER RELEVANT EXPERIENCE:}

\section{May - December $2013 \quad$ Science writer for eHow and LIVESTRONG}

I contributed articles to the Fitness sections of eHow and LIVESTRONG. I have written several articles about topics related to exercise physiology, muscle structure, and exercise-related injuries. My recent published articles include: Exercise and Homeostasis Activities, The effects of Short Period Exercise on Human Physiology, Evaluation of Maximum Muscular Strength, How do Floatation Devices Work 